\title{
Crush Strength Analysis of Hollow Glass Microspheres
}

\author{
Benjamin Dillinger \\ Thesis submitted to the faculty of the Virginia Polytechnic Institute and State University in \\ partial fulfillment of the requirements for the degree of \\ Masters of Science \\ In \\ Materials Science and Engineering
}

David E. Clark, Chair

Carlos T. A. Suchicital

George G. Wicks

$8 / 10 / 2016$

Blacksburg, Va

Key Words: Porous Wall Hollow Glass Microsphere, Nanoindentation, Crush Strength, Weibull Analysis, Microsphere Size Analysis 


\title{
Crush Strength Analysis of Hollow Glass Microspheres
}

\section{Benjamin Dillinger}

\begin{abstract}
$\underline{\text { Abstract }}$
Porous Wall Hollow Glass Microspheres (PWHGMs) were developed by the Savannah River National Laboratory. What makes these microspheres unique is the interconnected porosity spread throughout their wall allowing various materials to travel from the surface to the hollow interior. With their characteristic porosity, the PWHGMs are a great tool for encapsulating or filtrating different materials. Unfortunately, there is little information available on the mechanical properties of PWHGMs.
\end{abstract}

The main goal of this research was to develop a method to crush individual microspheres and statistically analyze the results. One objective towards completing this goal was to measure the microsphere diameter distribution. Microsphere diameter is a major factor affecting strength as well as the Weibull parameters. Two different methods, microscopy counting and laser light scattering, used in the research yielded similar distributions.

The main objective of this research was to analyze the crush strength of individual microspheres. Using nanoindentation, data were collected to analyze the crush strength of PWHGMs in uniaxial compression. Nanoindentation data were used to analyze how the strength of the PWHGMs changes through the different stages of production and at different diameter ranges. Data for 3M commercial microspheres were compared to ARC microspheres. Most data were analyzed using a statistical technique known as the two parameter Weibull analysis. The data indicated that the strength generally decreased as the microsphere diameter increased. Scattering in the data was nearly the same across all sample sets tested. Results indicated that the PWHGMs were weaker than the ARC hollow glass microspheres (HGMs). This is primarily due to the addition of wall porosity in the PWHGM. 


\section{$\underline{\text { Acknowledgements }}$}

I would like to thank Dr. David Clark, Dr. Carlos Suchicital, and Dr. George Wicks for being part of my Masters Committee. They have provided valuable help and advice. Working with them has been a valuable learning experience. I would like to thank the Applied Research Center (ARC) in South Carolina for providing me with microspheres used in the research and access their equipment. Additional thanks goes to Fred Humes for allowing me to work with the PWHGMs and Patrick Woodell for help with sample preparation and equipment training. I would also like to thank Jay Tuggle at the Nano Characterization and Fabrication Laboratory (NCFL) for providing advice on nanoindentation testing. I would like to thank the Materials Science and Engineering (MSE) and Biomedical Engineering and Mechanics (BEAM) departments at Virginia Tech for allowing me to use their equipment for testing. Finally I would like to thank my parents for their support while I worked on my Masters degree. 


\section{$\underline{\text { Table of Contents }}$}

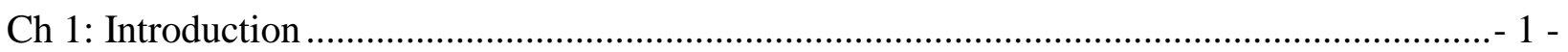

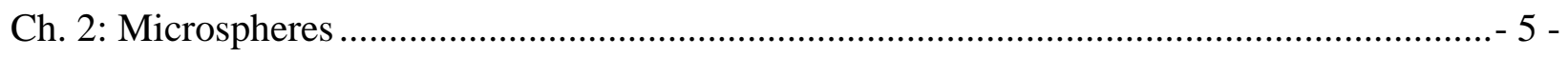

2.1: Porous Wall Hollow Glass Microsphere Production .................................................. 5 -

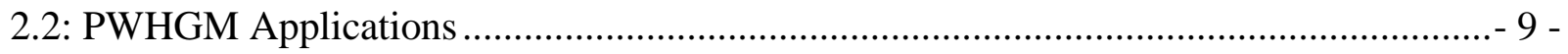

Ch. 3: Microsphere Size Analyses ................................................................................... 15 -

3.1: Laser Scattering Analysis of Microspheres.............................................................. 15 -

3.2: Microscope Counting Analysis of Microspheres ....................................................- 18 -

3.4 Microsphere Size Analyses Results ....................................................................... 21 -

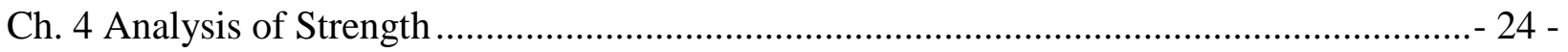

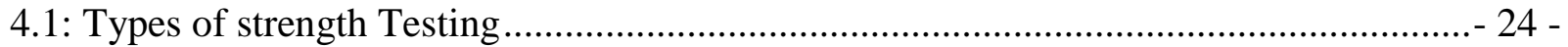

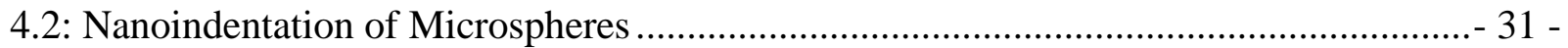

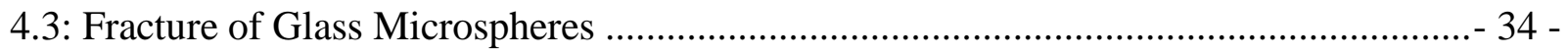

4.4: Weibull Analysis of Strength Data ................................................................. 36 -

Ch. 5: Experimental Design and Methods of Data Analysis .............................................. 43 -

5.1: Crush Strength Testing................................................................................... 43 -

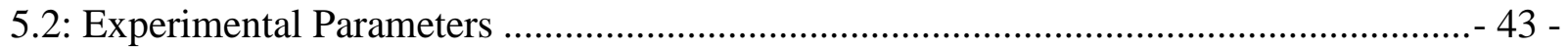

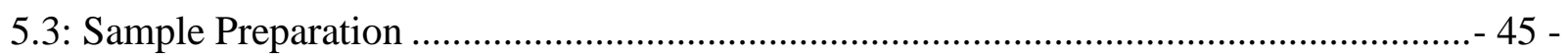

5.4: Nanoindentation of Microspheres ..................................................................... 48 -

5.4: Interpretation of Nano-Crush Test Results ........................................................- 52 -

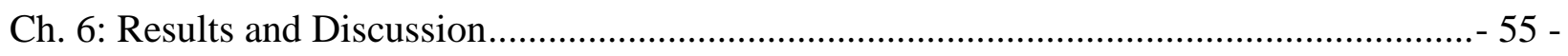

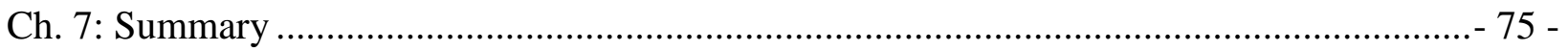

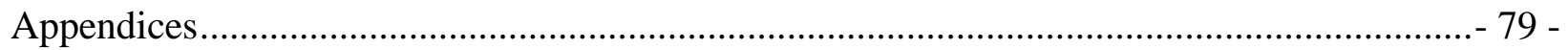

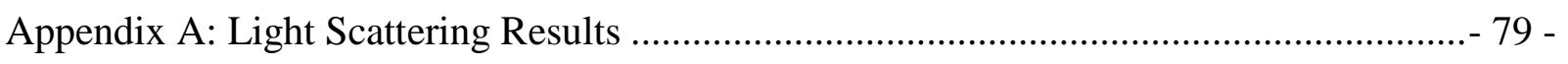

Appendix B: ARC Microscope Analyses and 3M Data..............................................- 86 -

Appendix C: Bar Graphs of the Numeric and Volumetric Fractions (\%) of Microspheres with

Specified Diameters Using Data Obtained from ARC's Microscope............................... 91 -

Appendix D: Additional Variations to Nanoindenter Results........................................ 94 -

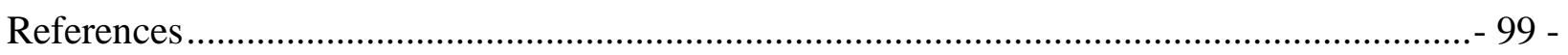




\section{$\underline{\text { Table of Figures }}$}

Figure 1: ARC Hollow Glass Microspheres Compared to a Human Hair. ............................... 2 -

Figure 2: Hollow Glass Microsphere Wall beside Human Hair ........................................... 3 -

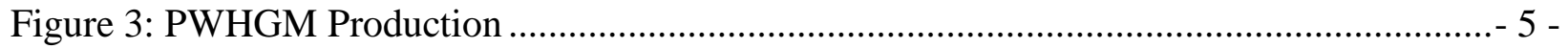

Figure 4: Detail of Porous Wall .......................................................................................... 8 -

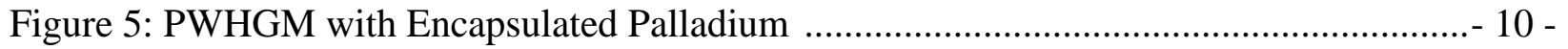

Figure 6: Sodium Alanate Encapsulated in a PWHGM ...................................................... 11 -

Figure 7: Molecule Retention Before and After Washing ................................................ 13 -

Figure 8: Time Release of IgG (A); Time Release of the Nucleic acid Cy3 Gated with Dextran

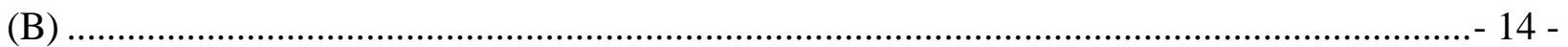

Figure 9: Static Light Scattering Example from LS Instruments .....................................- 16 -

Figure 10: Laser Scattering Results for One S60HS Test................................................. 18 -

Figure 11: Number and Volume Microsphere Size Distribution for 3M's S60HS Microspheres

Based on Microscope Analysis ......................................................................................... 21 -

Figure 12: Microsphere Breakage Curve .................................................................. 26 -

Figure 13: Uniaxial Tension Test ..................................................................................... 27 -

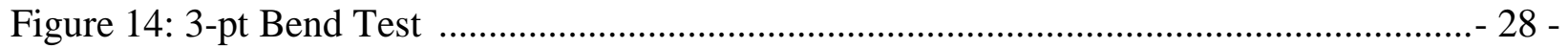

Figure 15: Two Dimensional Representation of a Thin Walled Microsphere Illustrating Proposed

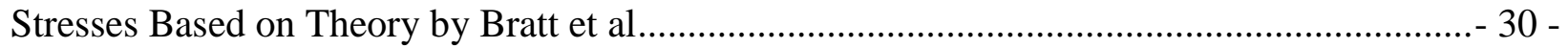

Figure 16: Typical Compression Test............................................................................... 31 -

Figure 17: Nano Crush Test Results for Koopman Et Al ...............................................- 32 -

Figure 18: Different Types of Nano Crush Test Results ...................................................... 33 -

Figure 19: Flaw Size and Number Distribution between Different Samples .........................- 37 -

Figure 20: Two Parameter Weibull Plot Showing how the Weibull Parameters are Determined

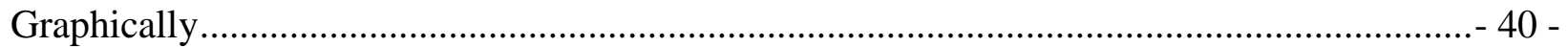

Figure 21: Fused Silica Slide that is Labeled and marked ............................................... 46 -

Figure 22: Container of Microspheres ............................................................................ 46 -

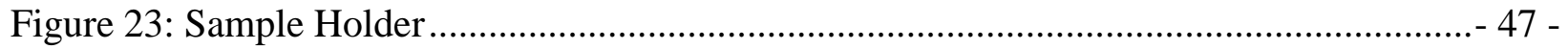

Figure 24: Nanoindenter Sample Schematic................................................................. 48 -

Figure 25: Microsphere Illustrating the Impression Left by the Nanoindenter Sharp Tip Before

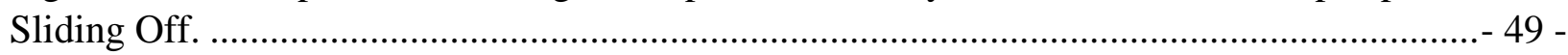

Figure 26: Schematic of a Flat Tip Nanoindenter Crush Test ..........................................- 50 -

Figure 27: Indentation Experiment Type ........................................................................... 51 -

Figure 28: Typical Nanoindenter Test Results (3M S38HS Sample)................................... 53 -

Figure 29: Microsphere before Test (A); Broken Microsphere After Test (B) ...................... 55 -

Figure 30: Normal Nano Crush Test Result .................................................................... 56 -

Figure 31: Microsphere Initially Moving Through Grease................................................ 56 -

Figure 32: Measured PWHGM Half-Shell (A); Measured PWHGM Wall (B) ......................- 58 -

Figure 33: ARC HGM Weibull Analysis......................................................................... 62 -

Figure 34: Heat Treated ARC HGM Weibull Analysis........................................................ 63 -

Figure 35: ARC PWHGM Weibull Analysis.............................................................. 63 - 
Figure 36: ARC PWHGM Three Parameter Weibull Analysis Using $\sigma_{\mu}=270$ psi vs. Two

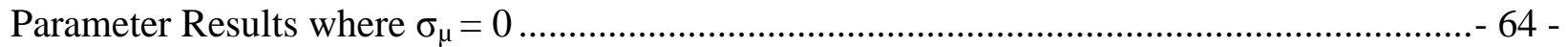

Figure 37: ARC PWHGM Three Parameter Weibull Analysis Using $\sigma_{\mu}=300$ psi vs. Two

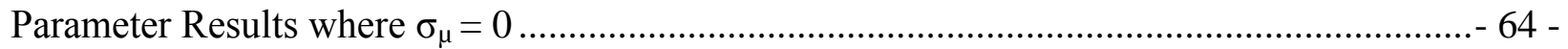

Figure 38: PWHGMs Calculated Using Equation (7) ...................................................- 65 -

Figure 39: Effects of the Different Stages of PWHGM Production on Strength for 30-40 Micron

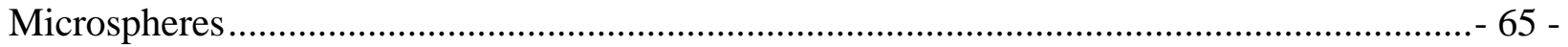

Figure 40: Effects of the Different Stages of PWHGM Production on Strength for 45-55 Micron

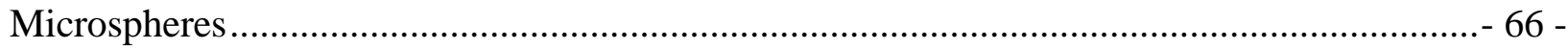

Figure 41: Effects of the Different Stages of PWHGM Production on Strength for 60-70 Micron

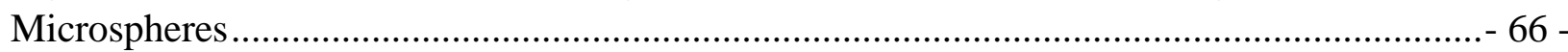

Figure 42: 3M S38HS and S60HS Microspheres ............................................................ 67 -

Figure 43: Effects of Different Stages of Production on Combined Data Sets .....................- 70 -

Figure 44: 35 Micron PWHGM Data Used to Predict Strength of PWHGMs at 70 Microns and

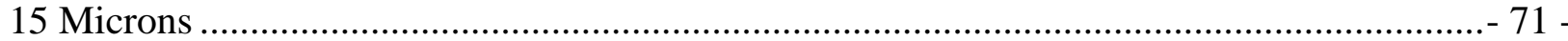

Figure 45: ARC HGM Laser Scattering Results Showing 5 Samples from the Same Batch with

Each Sample Run Twice .......................................................................................... 80 -

Figure 46: S38HS Test 1-5 Laser Scattering Results Showing 5 Samples from the Same Batch

with Each Sample Run Twice .................................................................................... 81 -

Figure 47: S38HS Test 6-10 Laser Scattering Results Showing 5 Samples from the Same Batch

with Each Sample Run Twice ............................................................................................ 82 -

Figure 48: S60HS Laser Scattering Results Showing 5 Samples from the Same Batch with Each

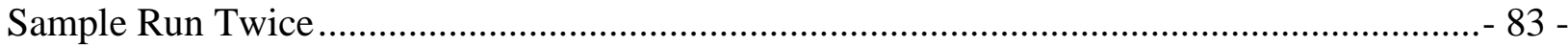

Figure 49: IM16K Laser Scattering Results Showing 5 Samples from the Same Batch with Each

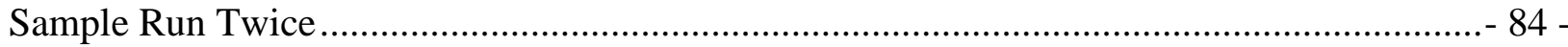

Figure 50: IM30K Laser Scattering Results Showing 5 Samples from the Same Batch with Each

Sample Run Twice ............................................................................................ 85 -

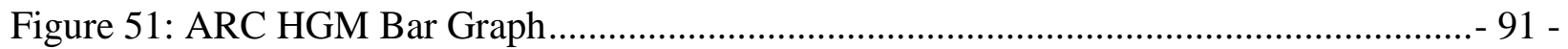

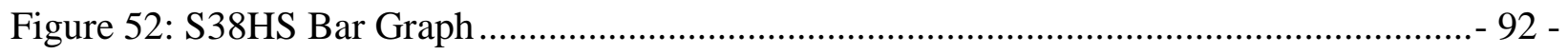

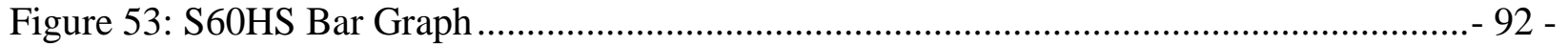

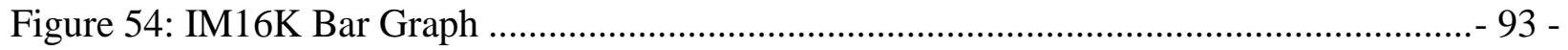

Figure 55: IM30K Bar Graph ................................................................................... 93 -

Figure 56: Microsphere after Test where Not Many Data Points were Recorded................... 95 -

Figure 57: Initial Increase in Load Required Before Deformation Will Occur....................... 96 -

Figure 58: Small Increments Where Deformation Occurs Without an Increase in Load ......... 96 -

Figure 59: Initial Point of Fracture Followed by Region Requiring More Force to Cause

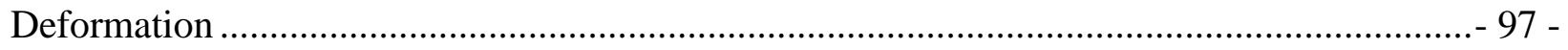

Figure 60: Inconsistent Slope in Region before Fracture ................................................ 97 -

Figure 61: Test Result Where Test Occurred Faster than Output Could be Recorded .............- 98 -

Figure 62: Nanoindenter Recorded Additional Data While Tip Was Removed From Sample - 98 - 


\section{Table of Tables}

Table 1: S60HS Combined Table Showing Both Numeric and Volumetric Distribution Data- 20 Table 2: Combined Volume Distribution Table for Microsphere Size Distribution. ................ 23 Table 3: Microsphere Diameter and Corresponding Wall Thickness ........................................ 59 Table 4: 3M S38HS Microsphere Diameter and Corresponding Wall Thickness ....................... 60 Table 5: 3M S50HS Microsphere Diameter and Corresponding Wall Thickness ....................... 60 Table 6: Calculation of Probability of Failure ...................................................................... 61 Table 7: Weibull Analyses for Each Data Set ....................................................................... 68 Table 8: Published vs. ARC Measured Distributions for 3M Microspheres .............................- 87 Table 9: Numeric Distribution for 3M and ARC Microspheres ............................................. 87 -

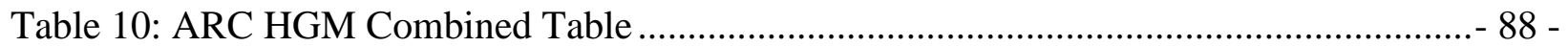

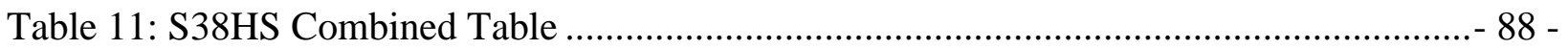

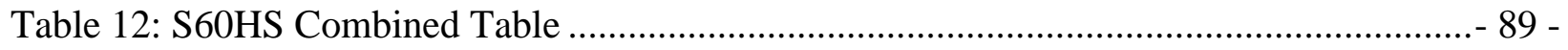

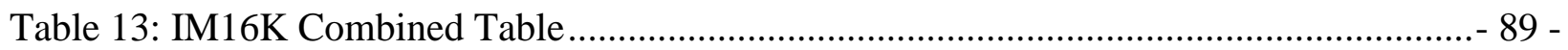

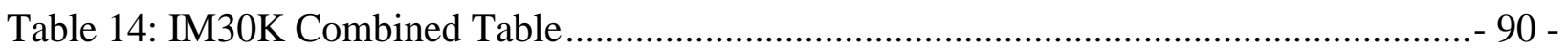




\section{Ch 1: Introduction}

Microspheres are small spheres made of various types of materials that are usually less than 100 microns in diameter. These microspheres can be hollow or solid and have been made from a variety of materials including metals, polymers, and ceramics/ glasses. Hollow Glass Microspheres (HGMs) were developed by 3M in the 1960s and originally used for buoyancy applications [1]. Later, 3M used microspheres as strong, light weight fillers for a variety of products ranging from foams to coatings. HGMs have found use in a variety of other applications from defense to transportation to construction [1], [2]. Around 2005, a new type of glass microsphere was developed at the Savannah River National Laboratory (SRNL) by George Wicks, Ray Schumacher, and Kit Heung [3]. This new hollow glass microsphere was unique due to the development of interconnected porosity in the glass wall of the microsphere and is known as a Porous Wall Hollow Glass Microsphere (PWHGM). The addition of pores in the microsphere makes it possible to fill the hollow cavity of the PWHGM with different materials. This development creates many new applications for filtration and encapsulation using PWHGMs [4], [5], [6], [7].

The basic properties of PWHGMs are different compared to other hollow glass microspheres. PWHGMs can have a diameter that ranges from 2 to 100 microns. This is smaller than a human hair, which is around 100 microns in diameter, as seen in Figures 1 and 2. PWHGMs have wall thicknesses in the range of approximately 0.5 to 2 microns giving them a diameter to wall thickness ratio range between 4 and 200. By definition thin walled structures have diameter to wall thickness ratios of greater than 10 [8], [9]. Unlike many commercial microspheres, such as the soda lime borosilicate ones manufactured by 3M, PWHGMs are comprised of around $96 \%$ pure silica. The method used to produce the microspheres is a 
combination of 3M's microsphere production method and Corning's method for producing Vycor glass. PWHGMs start out as borosilicate HGMs and then are heat treated. Due to their composition, these HGMs undergo spinodal decomposition and phase separate into two interconnecting phases: sodium borate and silica. The sodium borate is leached away with acid leaving mostly porous silica as the wall material. This is how the characteristic nanoporosity of the PWHGMs is created. The wall porosity will generally be 0.01 to 0.1 microns in diameter. Encasing the wall are two layers that are thought to be created during the leaching step. These layers exhibit a different type of porosity than the inner wall porosity [4], [5], [6], [7].

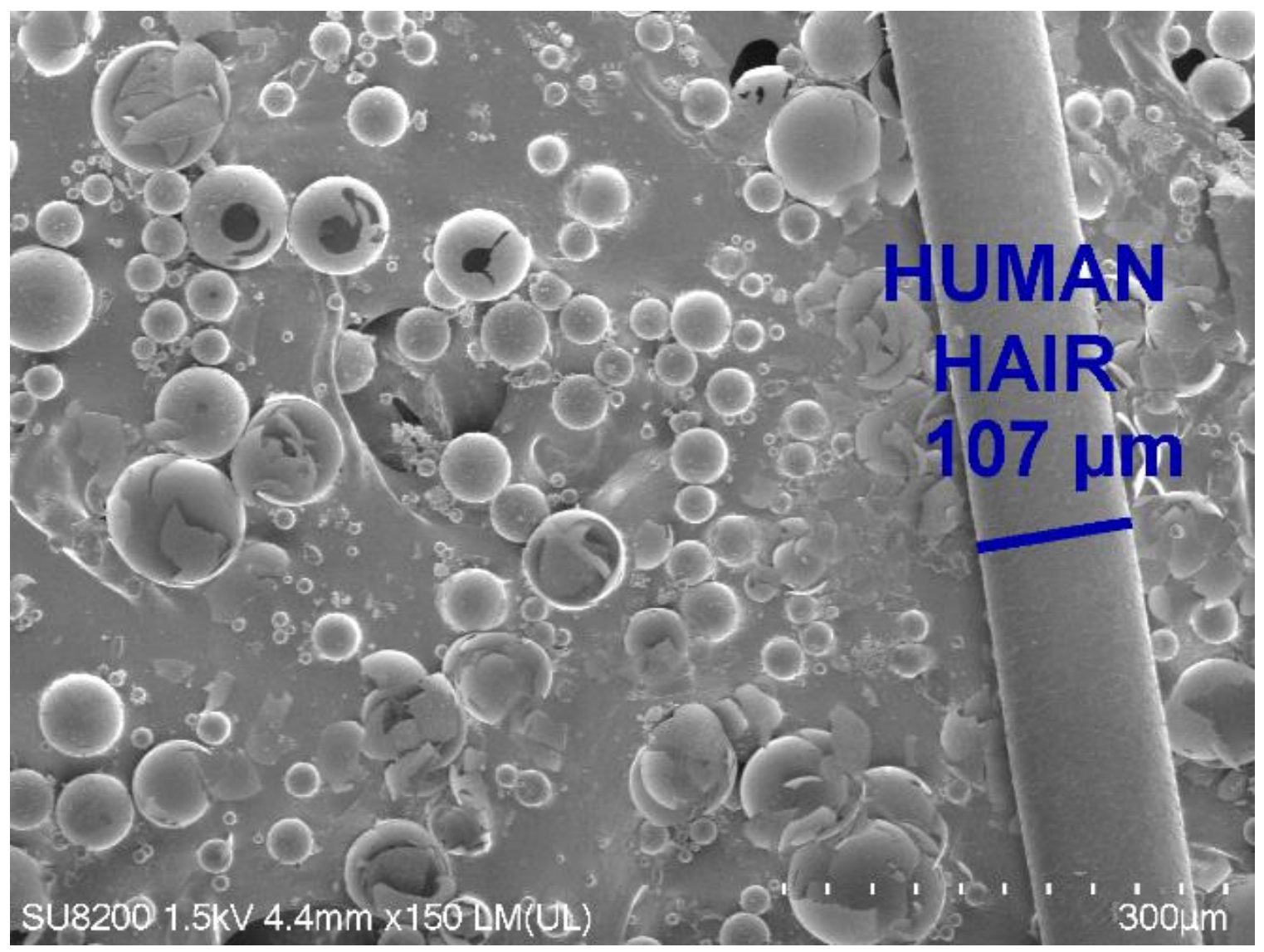

Figure 1: ARC Hollow Glass Microspheres Compared to a Human Hair. The majority of these were deliberately broken prior to SEM analysis. 


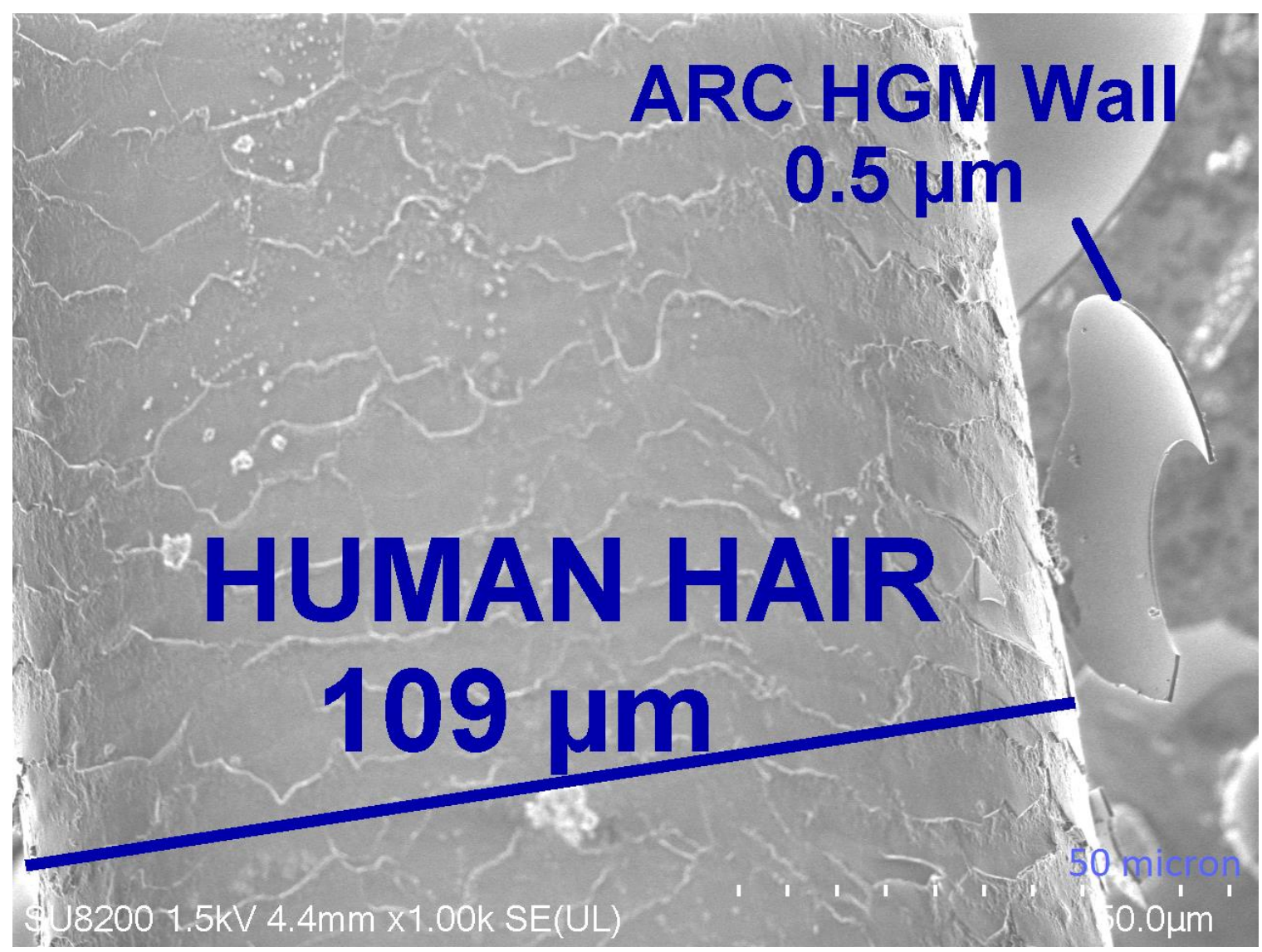

Figure 2: Hollow Glass Microsphere Wall beside Human Hair

Currently, most research on these unique microspheres is conducted through the Applied Research Center in South Carolina (ARC). The majority of research done through ARC examines the PWHGMs ability to encapsulate and filter various materials using its nanoporosity. These applications include encapsulating medicine for drug delivery, storing metal hydrides for hydrogen fuel storage, MRI enhancements, and the encapsulation of palladium for hydrogen isotopes. In all of these applications, the strength of the microspheres is an extremely important property [4], [5], [6], [7].

Unfortunately, there has been little research done to examine the strength of the microspheres. Due to their size and shape it is extremely difficult to accurately measure and analyze the strength of the microspheres. Strength data provided by manufacturers of 
microspheres are usually determined by testing large quantities of microspheres at once and are primarily meant to be used for quality control purposes. A literature search shows that measuring the crush strength of an individual microsphere is a nontrivial problem. Of the several approaches that have been suggested, nanoindentation using a flat end tip is the most plausible method.

One objective for this research was to accurately measure the microsphere diameter distribution since microsphere size plays a significant role in strength. The objective was accomplished using both laser light scattering and microscopy counting techniques.

The main goal of this research was to crush individual microspheres using nanoindentation with a flat end tip and analyze the data using Weibull analysis to determine characteristic strengths and range of strength scattering. Numerous microspheres underwent indentation to create data sets for multiple types of microspheres. For the majority of the tests, the two parameter Weibull analysis was used to analyze the scattering in the data and determine the crush strength of the majority of microspheres. A few tests were analyzed with a three parameter Weibull analysis. With the Weibull analyses the strength of PWHGMs were analyzed as a function of diameter and stage of production. 


\section{Ch. 2: Microspheres}

\section{1: Porous Wall Hollow Glass Microsphere Production}

PWHGM production combines the methods used by $3 \mathrm{M}$ and Corning for their Hollow Glass Microspheres and Vycor glass, respectively [7]. Detailed current production methods are protected IP, but the general experimental method used to fabricate the initial PWHGMs for research is published and described below. Figure 3 outlines the individual steps for the process.

\section{Porous Wall Hollow Glass Microsphere Production Process}

$\begin{array}{ll}\text { Step } 1 & \begin{array}{l}\text { Melt Raw Materials into } \\ \text { Desired Composition } \\ \text { with Sulfate Blowing } \\ \text { Agent } \\ \text { Quench, Crush, and Sieve } \\ \text { Glass into Frit with a } \\ \text { Diameter of } \leq 45 \text { Micron } \\ \text { Step } 2\end{array} \\ \text { Step } 3 & \begin{array}{l}\text { Flkali Borosilicate Glass } \\ \text { Blowing agent } \\ \text { Melt Glass and Decompose } \\ \text { Blowing Agent to Form } \\ \text { Microspheres } \\ \text { Alkali Borosilicate } \\ \text { Microsphere }\end{array} \\ \text { Step 5 } & \begin{array}{l}\text { Heat Treatment } \\ \text { Phase Separated } \\ \text { Microsphere } \\ \text { Acid Leaching }\end{array}\end{array}$

Figure 3: PWHGM Production 
In Step 1, the raw materials and sulfate blowing agent are melted to form an alkali borosilicate liquid at a temperature around $1200-1300^{\circ} \mathrm{C}$ for $2-3$ hours. This temperature is lower than the decomposition temperature of the blowing agent but high enough to melt the raw material. Step 2, the liquid is cooled to form a glass that is crushed and ground into a frit and sieved to a size of 45 microns or less using a 325 mesh screen. Step 3, the glass particles are again sent though a flame, this time at a temperature higher than $1300^{\circ} \mathrm{C}$ so the blowing agent will decompose and create gas inside the particles. This gas causes the particles to form into hollow spheres. Water sprays are used to cool and collect the microspheres. Due to their hollow nature, the HGMs are about $1 / 2$ as dense as water and float while solid spheres and glass shards sink. The HGMs are collected and filtered with 6 micron filter paper and anything that has sunk is collected later [4].

At this point the microspheres are made of nonporous alkali borosilicate glass. To get the final product the HGMs must be heat treated to cause phase separation and then leached. Due to their composition, the HGMs will enter a miscibility gap and phase separate when heat treated. By heat treating at over $500^{\circ} \mathrm{C}$ for longer than 6 hours in Step 4, the borosilicate glass will transform into two continuous phases comprised of mainly high purity silica and sodium borate. The driving force for the phase separation is spinodal decomposition [4]. Spinodal decomposition is a continuous type of phase transition that occurs at a composition miscibility gap in a phase diagram. In the miscibility gap, a material will be more stable separating into two phases instead of continuing to exist as one phase. This occurs because the Gibbs free energy of the solution is higher than the individual phases [10]. Heat treating the glass will also relieve internal stresses produced during production. The difference in the thermal expansion 
coefficients between the two phases could potentially cause new internal stresses to form in the microsphere during cooling [11], [12].

After the heat treatment is complete, the microspheres are immersed in a solution, usually hydrochloric acid, for Step 5. In this step the sodium borate phase will react with the acid and be leached out leaving the silica phase intact. Once leaching is complete, the microspheres will have porous walls that allow material to transport between the hollow interior and external surface. Microspheres that were correctly leached will sink as they fill with hydrochloric acid while those that do not leach correctly will still float. Afterwards, the microspheres are washed in water multiple times to remove the hydrochloric acid and then dried at $100^{\circ} \mathrm{C}$ [4]. During the leaching and washing steps, the sodium borate will not be removed completely. This remaining sodium borate creates porous surrounding layers around the silica glass wall as seen in Figure 4 A. Porosity in the surrounding layers is different from the wall porosity and has an average diameter 0.1 microns compared to the internal porosity diameters of 0.01 to 0.1 microns as seen in Figure 4 B. 

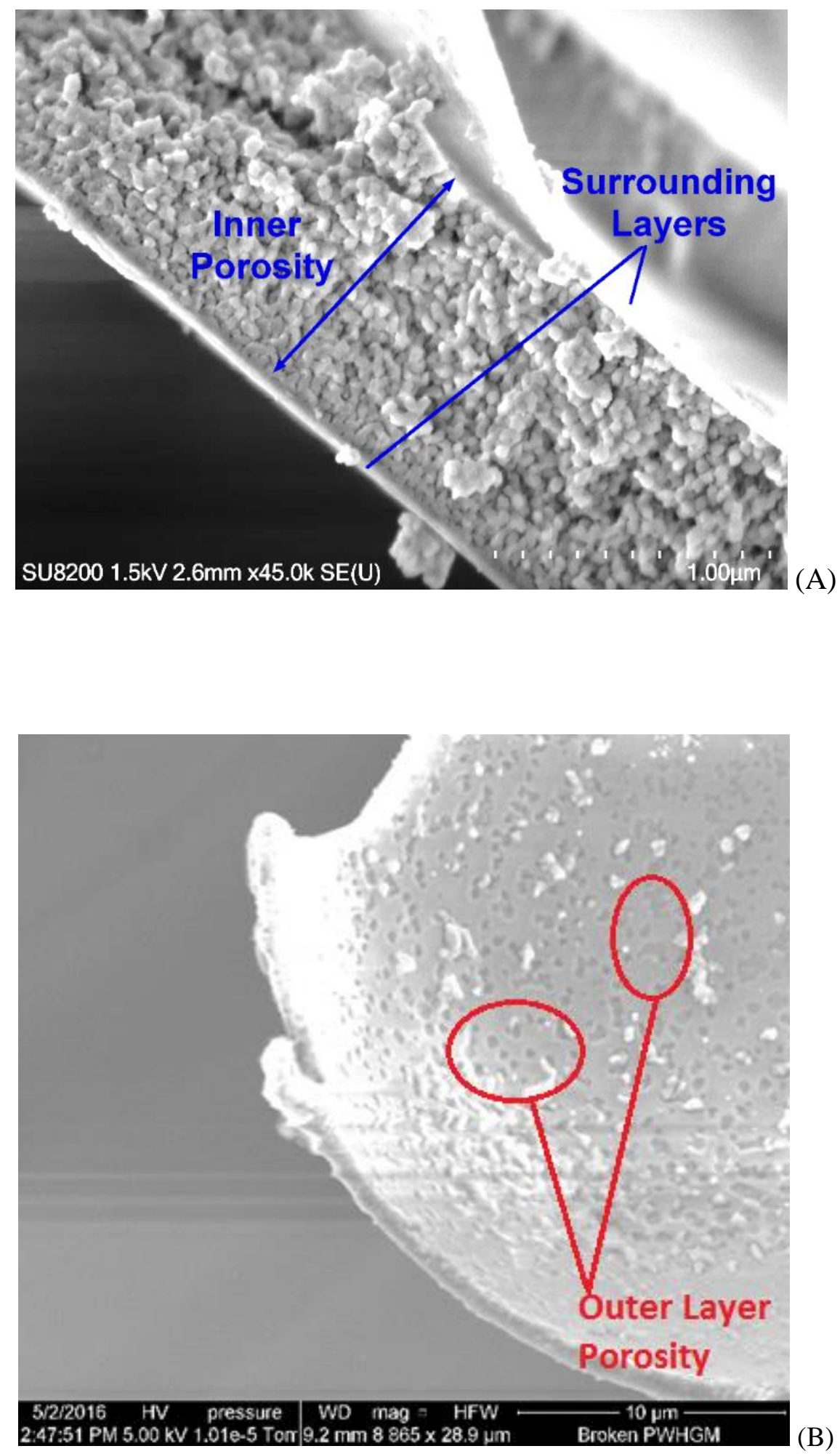

(B)

Figure 4: Detail of Porous Wall. Side View of PWHGM Wall (A); Outer Layer Porosity (B) 


\section{2: PWHGM Applications}

There are a variety of applications for PWHGMs that have been published. Some of the early applications involve using microspheres for hydrogen fuel and separation purposes [4], [5]. Most recent applications involve encapsulating different materials with medical purposes into PWHGMs while the most recent use is in security inks and anti-counterfeiting activities [6].

One of the first areas of PWHGM research involved encapsulating palladium into PWHGMs. One property of palladium is to reversibly absorb the different isotopes of hydrogen. Palladium does not absorb the three hydrogen isotopes equally though; it absorbs protium more easily than deuterium and deuterium more easily than tritium. In hydrogen isotope separation, palladium is deposited onto porous granules for support and packed into long columns. This setup separates the different types of hydrogen in a way similar to how chromatography works. The efficiency of this method depends on how well the palladium is packed together. Palladium was encapsulated into PWHGMs as a saturated salt solution. Scanning electron microscopy (SEM) and electron dispersive $\mathrm{x}$-ray spectroscopy (EDX) were used to examine the results of the encapsulation. Intact microspheres were identified using the SEM and then broken between a metal plate and the substrate. Afterwards the broken PWHGMs were examined again with SEM to find microspheres filled with palladium as seen in Figure 5. EDX was used to confirm that the encapsulated material was indeed palladium [4]. 


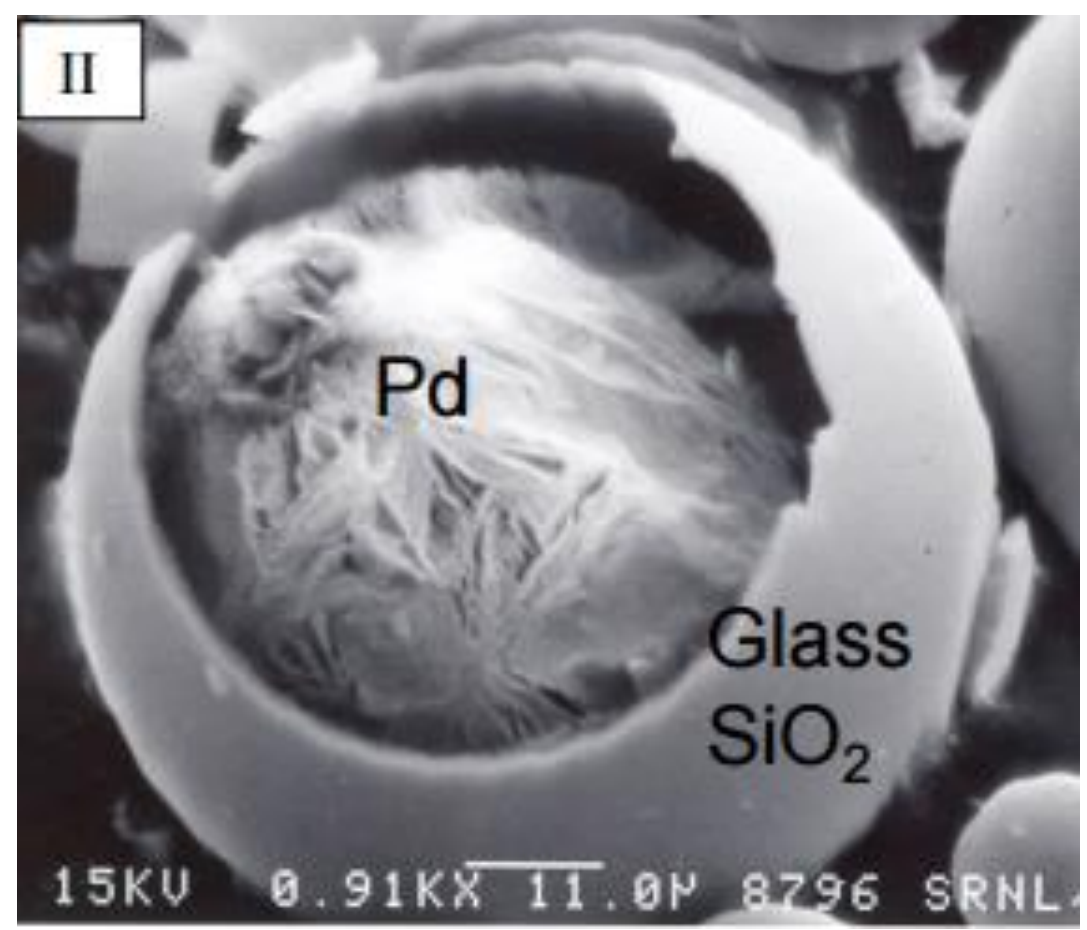

Figure 5: PWHGM with Encapsulated Palladium [7]

Another hydrogen based application for PWHGMs involves encapsulating complex metal hydrides that could be used to store hydrogen fuel. Current hydrogen fuel technology requires hydrogen to be stored either at high pressures or low temperatures to get the desired fuel density. This is not an optimal situation for storing and using hydrogen fuel. One alternative to using pure hydrogen as a fuel are complex metal hydrides. These materials were examined as an alternative because they could be stored at moderate temperatures and pressures unlike pure hydrogen and may provide better fuel density overall. Unfortunately, under ambient conditions metal hydrides react with air and water. Encapsulation of the metal hydrides in PWHGMs was determined to be one method that could prevent the materials from reacting with their surroundings by using the PWHGMs as a "micro-barrier [5], [13], [14], [15]. 
Sodium alanate was chosen as the metal hydride to be encapsulated. To examine the microspheres, a small amount of the filled PWHGMs were stabilized in air. The filled PWHGMs were broken open by applying slight pressure to the microspheres. Two types of structures were seen when examining the filled PWHGMs. In the interior of the microsphere the alanates formed a "bundled needle structure" while a bumpy formation was seen on the surface as seen in Figure 6. These structures are not representative of the normal formation for sodium alanate, so elemental mapping was conducted to confirm that the structures inside the PWHGMs were indeed the metal hydrides. The elemental mapping results showed the presence of sodium and aluminum in the PWHGM confirming the material was indeed sodium alanate. It was speculated that the porous silica provided the nucleation sites for the unique alanate structures [5], [13], [14], [15].

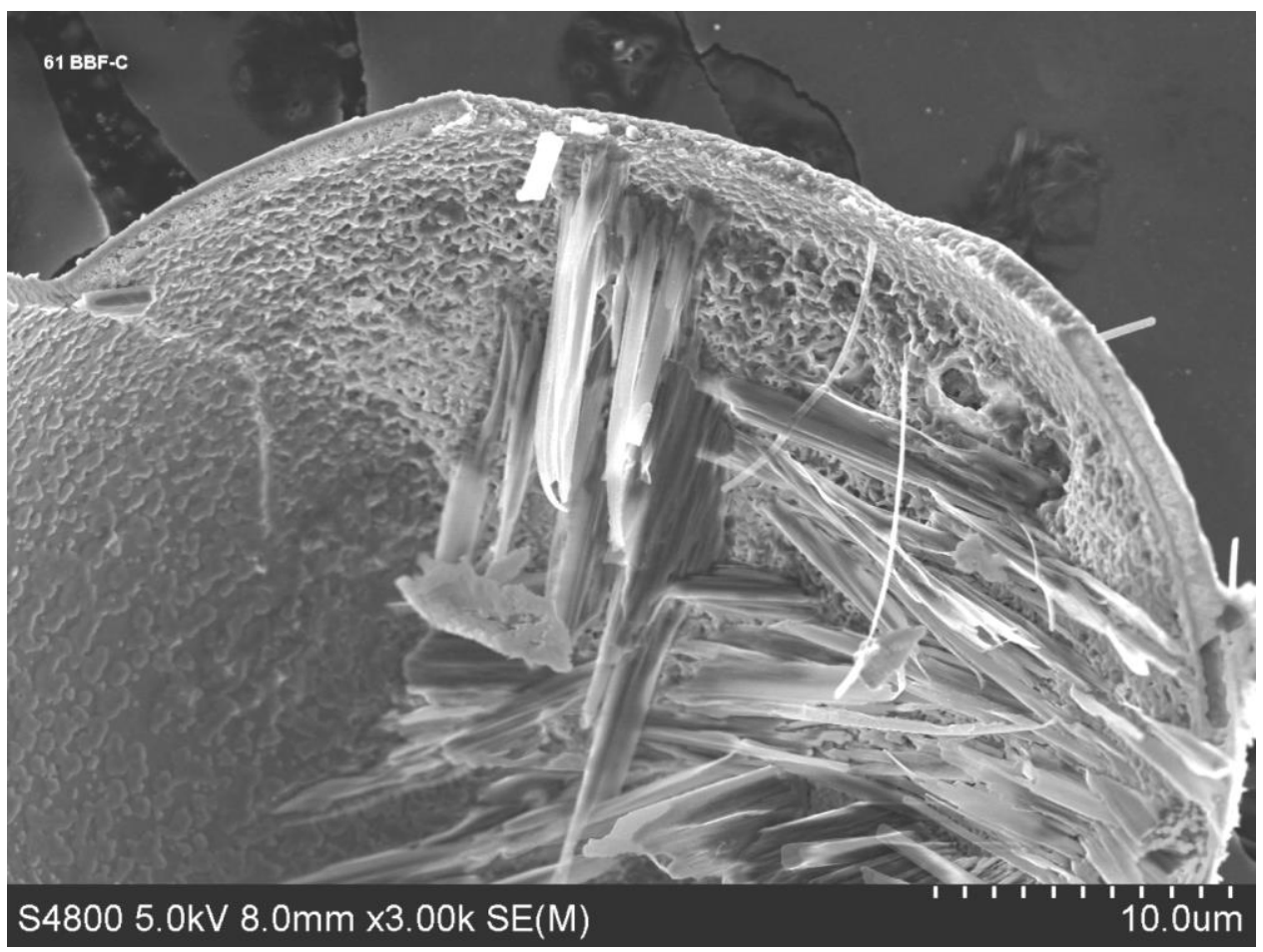

Figure 6: Sodium Alanate Encapsulated in a PWHGM [5] 
Glasses have been used in various biomedical applications such as hard tissue repair, dental crowns, and cancer treatment. One application that glass has not been used in is as a drug delivery platform. Collaboration between the Savannah River National Laboratory and multiple medical colleges examined encapsulating and releasing various biological molecules in PWHGMs for drug delivery. To determine the effectiveness of loading PWHGMs with different molecules, multiple forms of imaging were performed on loaded PWHGMs before and after they were washed. Dextran molecules, $500 \mathrm{kDa}(\sim 14.4 \mathrm{~nm})$ and larger, would not enter the PWHGM. Smaller dextran molecules would enter the PWHGMs, but were not retained after washing. 70 $\mathrm{kDa}$ dextran was unique as it was retained in the PWHGM wall at concentrations above those found in the solution as seen in Figure 7. Images of the PWHGMs loaded with various molecules were taken using a scanning confocal microscope [6], [16].

Multiple proteins were tested to identify which ones were retained after washing. Smaller proteins would enter and exit the PWHGMs freely like the smaller dextrans. The two largest proteins, immunoglobulin $\mathrm{G}(\mathrm{IgG})$ and conalbumin acted similar to the $70 \mathrm{kDa}$ dextran and were retained in the walls of the microspheres as seen in Figure 7. Because they filled the walls of the PWHGMs, the larger proteins retained part of the solution in the PWHGM cavity. Examining the protein loaded PHWGMs every two hours showed that the protein was slowly flowing out of the PWHGMs as seen in Figure 8 A [6], [16].

The final molecules loaded into the PWHGMs were nucleic acids. Like the smaller proteins and dextrans, the nucleic acids were not retained after washing. One experiment tried to retain the nucleic acids by loading them into the PWHGMs and then incubate the loaded PWHGMs in a solution containing $70 \mathrm{kDa}$ dextran. The dextran would coat the walls, allowing some of the PWHGMs to retain the nucleic acid as seen in Figure 7. Over time the nucleic acid 
signal would decrease as it was released from the PWHGM as seen in Figure 8 B. From the results it was determined that it is possible to use PWHGMs as a controlled delivery vehicle for medicine [6], [16].

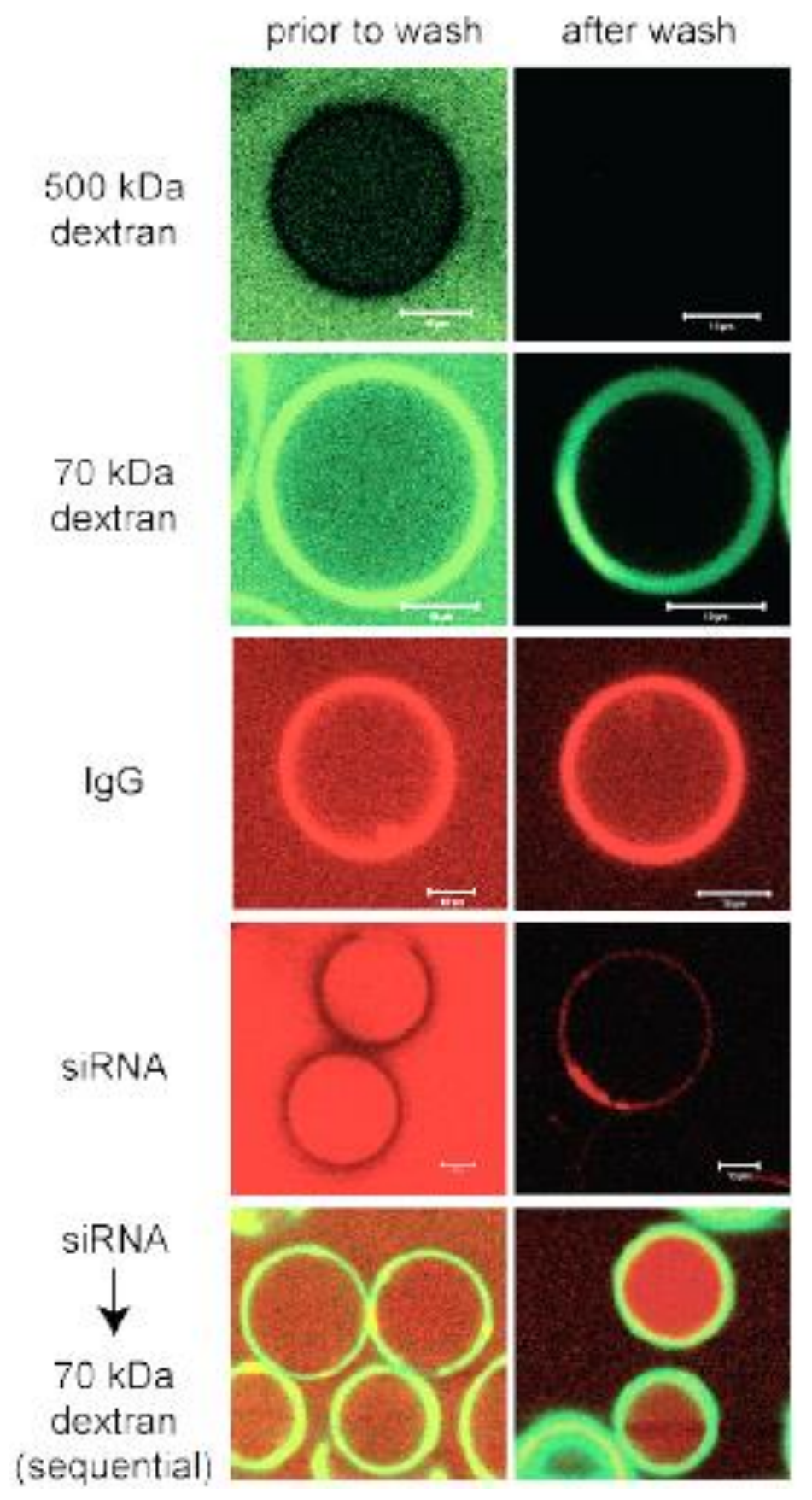

Figure 7: Molecule Retention Before and After Washing [16] 

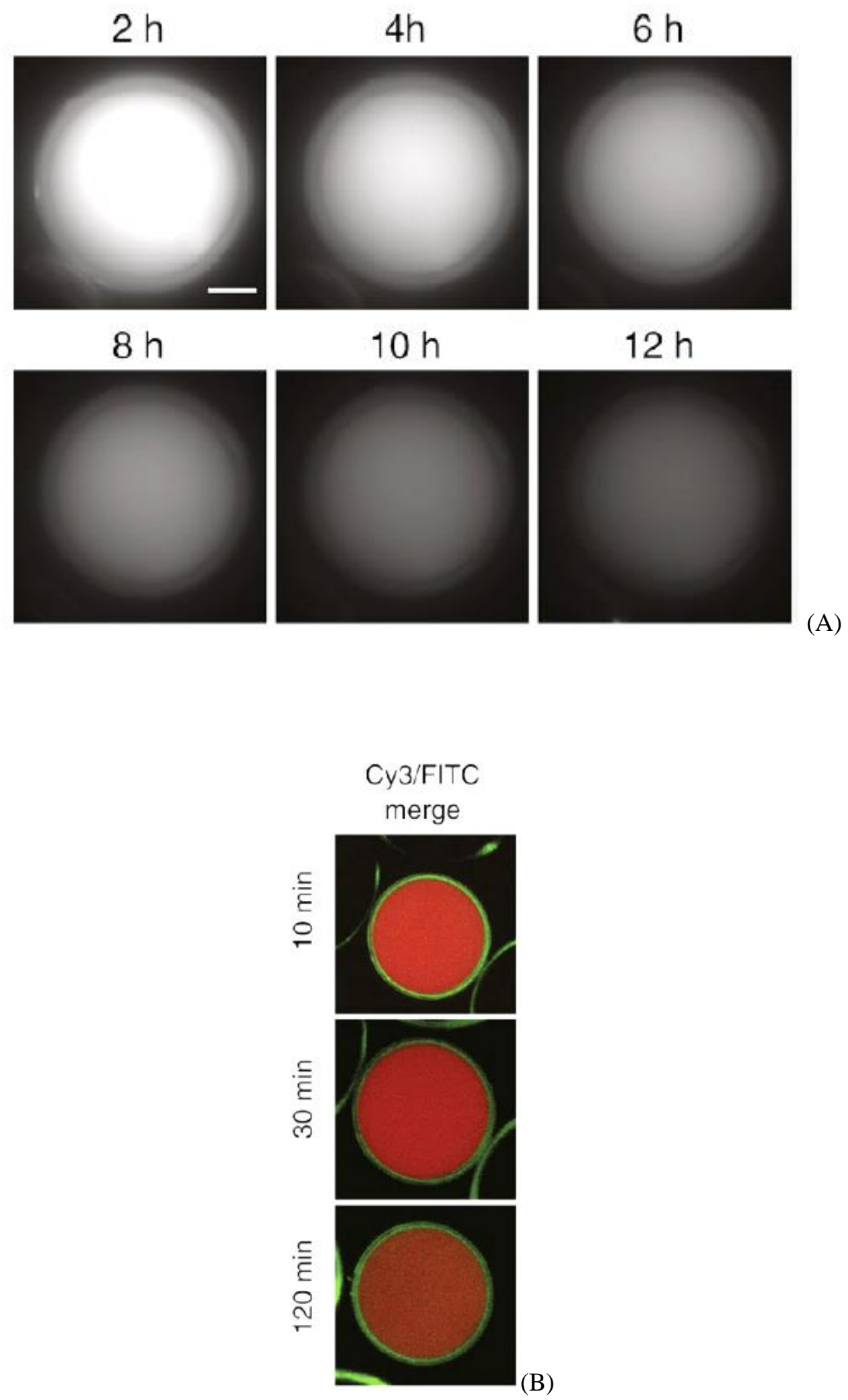

Figure 8: Time Release of IgG (A); Time Release of the Nucleic acid Cy3 Gated with Dextran (B) $[6]$ 


\section{Ch. 3: Microsphere Size Analyses}

As discussed earlier and also seen in Figure 1, the microspheres have a fairly broad range

of diameters. In order to evaluate the crushing strength of microspheres and the probability of failure under various levels of stress, it is necessary to know the microsphere size distribution. The two methods used in this research were laser light scattering and microscopy counting.

\section{1: Laser Scattering Analysis of Microspheres}

Laser light scattering calculates the size distribution of a powder by illuminating a suspension containing the powder with a monochromatic beam of light of known intensity and wavelength. The particles scatter the light in various directions and the scattered intensity is measured at different angles using a detector as shown in Figure 9 [17]. The intensity of the scattered light, $I_{s}$, vs a scattering variable, $q$, are plotted on a Guinier plot as the $\mathrm{y}$ and $\mathrm{x}$ axes, respectively. Scattered light is measured with a detector using a light scattering theory to interpret the results. Light scattering theories take into account the various parameters that would affect the scattered light such as the refractive index of the particle and the solvent. The scattering variable is calculated using the wavelength of the laser light, the refractive index of the solvent, and the angle of scattering [18], [19], [20]. 


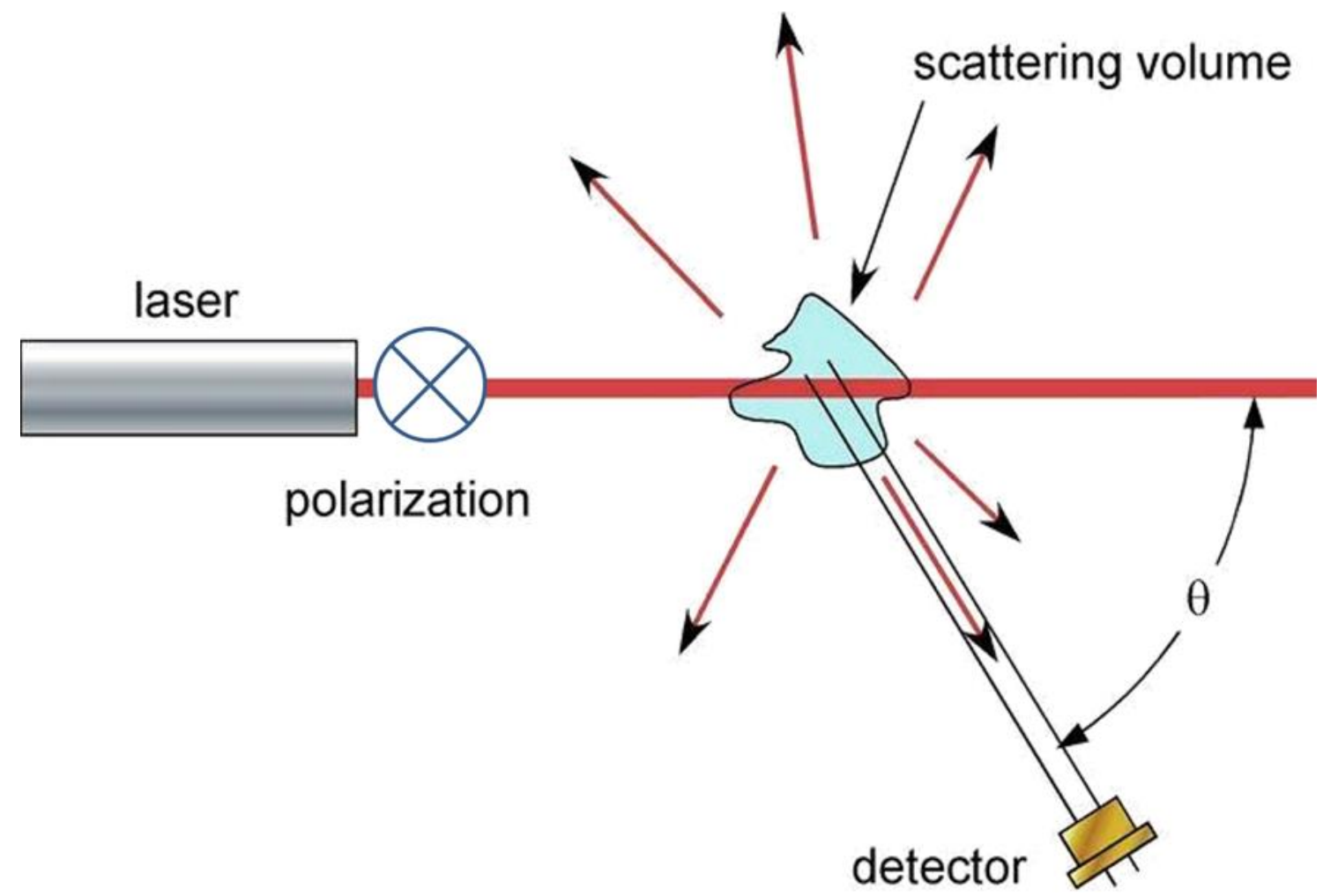

Figure 9: Static Light Scattering Example from LS Instruments [17]

The size distribution of four different types of 3M's microspheres and ARC's HGMs were tested using the Horiba LA950. This instrument uses laser light scattering to determine the microsphere size distribution. Each series had five individual samples tested and each sample had an additional test run on it after intensive ultrasounding. The suspension media was distilled water. The index of refraction used for the samples was 1.46 , which is value for silica and 0.01 different from borosilicate glass [10]. The solvent media was distilled water which has an index of refraction of 1.33 [21]. Before testing of a series began, the instrument was rinsed to clean out any leftover residue from prior tests. The water was debubbled to remove any trapped air bubbles in the solution that could have affected the results. The system was then blanked, which uses a test result of pure water to reset the transmittance readings for the system. Circulation was turned on to force the microspheres to mix instead of floating on top of the water. Before 
measuring the size distribution, ultrasound was run using minimal settings for 10 seconds to attempt to break up any microspheres that were agglomerated in the suspension. Once the ultrasound was completed, the sample measurement was taken. After the data from the first test was recorded, a second ultrasound, set at max settings for 30 seconds, was run to break up any microspheres still agglomerated and to see if the microspheres started to break from the force of the ultrasound. A second test on the same sample was recorded once the ultrasound was complete. With the completion of the second test, water was drained from the unit and refilled before starting another sample. There were no noticeable changes in the microsphere size distribution due to ultrasounding.

In each analysis there were two curves graphed. One curve was the probability density curve (PDF), which shows what percentage, $\mathrm{q} \%$, based on volume, of the microspheres is at a specific diameter. This curve was created using data recorded in a histogram. Each region of the histogram represents one part of 93 predetermined diameter ranges and the total percentage of the distribution will add to $100 \%$. The PDF is made by extrapolating a curve through the average diameter of each diameter range [22]. The second curve is the cumulative distribution function $(\mathrm{CDF})$ of the microsphere sample, which shows the probability that the microspheres had a diameter less than or equal to a specific size, undersize(\%). Both curves are related meaning that one curve can be determined if the other curve is known. This is because the PDF is the derivative of the CDF. Figure 10 shows a typical result from a laser scattering test. Notice that this is a bimodal distribution with the largest percentage ( 90\%) ranging from 10 to 70 microns and the smallest percentage $(\sim 10 \%)$ ranging from 0.2 to 0.8 microns. The total percentage under the curve adds to $100 \%$. Most likely the smaller size range represents debris. In addition to the graph, each analysis calculates the mean, median, mode, standard deviation, 
$10 \%, 50 \%$, and $90 \%$ results for the total distribution. The mean, median, mode, and standard deviation for the S60HS microspheres shown in Figure 10 are $23.87 \mu \mathrm{m}, 24.92 \mu \mathrm{m}, 27.00 \mu \mathrm{m}$, and $13.00 \mu \mathrm{m}$. Appendix A provides the combined results for each type of microsphere.

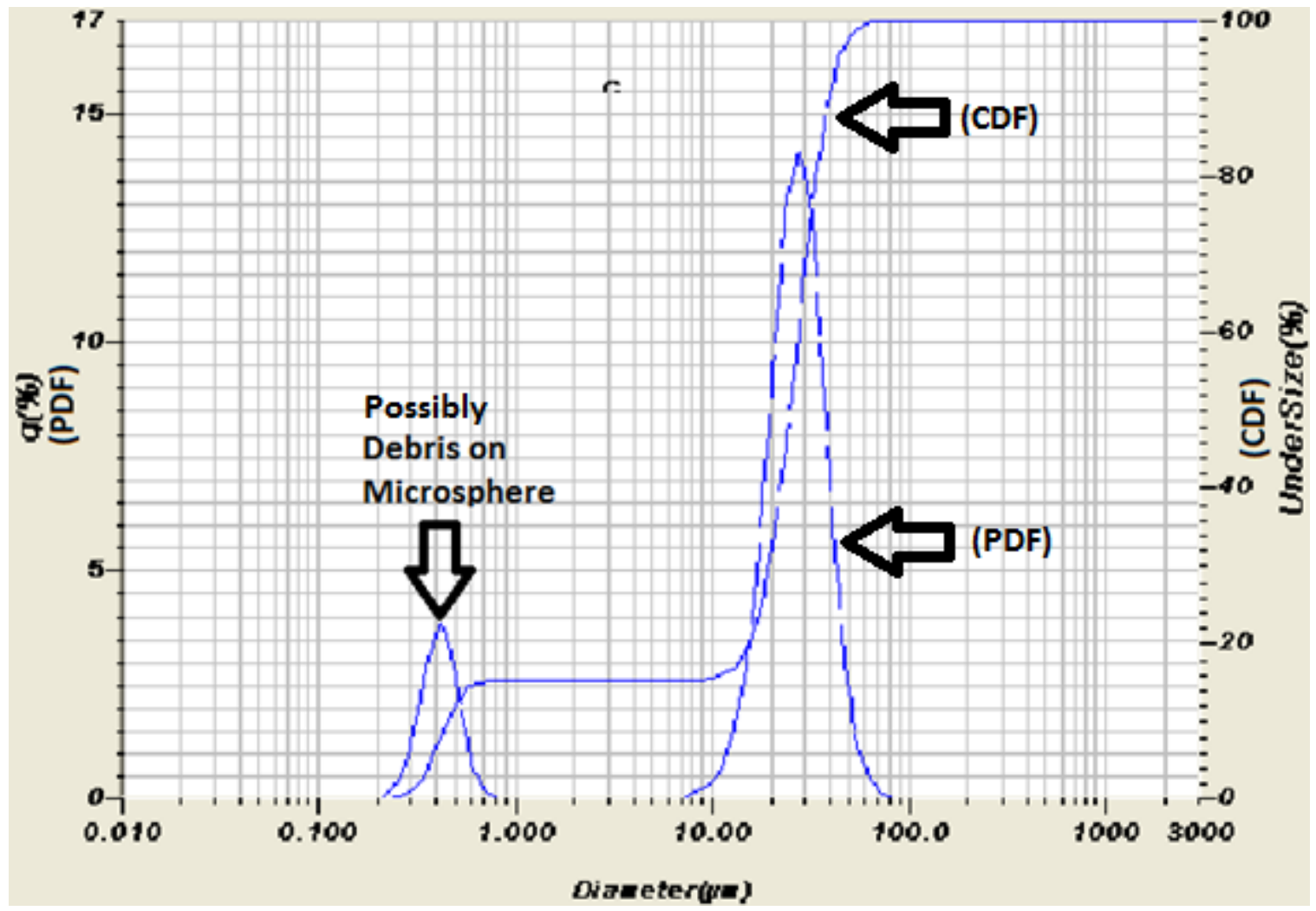

Figure 10: Laser Scattering Results for One S60HS Test

\section{2: Microscope Counting Analysis of Microspheres}

Microscopy counting analysis was conducted at ARC using a Zeiss Imager.A2M microscope. The Zeiss microscope was able to magnify samples between 5x and 1000x and examine them using bright field, dark field, and polarizers. Recorded images were analyzed using the Axiovision software package accompanying the instrument. Axiovision has the ability to distinguish between individual microspheres and measure their diameter using variations in the color and brightness of the objects in the image and the background. Results from this 
analysis were recorded as an output image labeling each microsphere and a table that displayed the measured values. The software then used the table to conduct a statistical analysis of the data. Multiple tables from different images could be combined to analyze a larger sample size. Microspheres analyzed were part of a dilute suspension that kept microspheres separated. Due to the way the microscope identified objects based on color and lighting, separating the microspheres made it easier for the software to identify the individual microspheres.

Samples for the analyses were made by sandwiching a microsphere filled suspension between a microscope slide and a cover slip. The suspension was made by mixing between 0.005 and 0.015 grams of microspheres with 2.4 to $3.2 \mathrm{~mL}$ of DI water for $3 \mathrm{M}$ products. For ARC HGMs oil was used instead of water to prevent the larger microspheres from breaking when the cover slip was put on. It also kept the microspheres in suspension longer as it was harder for microspheres to float to the top in the viscus liquid. The optimal suspension contained $0.8 \mathrm{~mL}$ of liquid and less than $0.010 \mathrm{~g}$ of microspheres and was kept in a small vial as this made it easier to remove later. Since the microspheres have a lower apparent density than the liquid they would quickly float to the top of the suspension. Larger microspheres float faster as they are less dense than smaller microspheres of similar wall thicknesses. To counter this, the suspension was constantly agitated to keep the microspheres mixed. This did not completely guarantee that samples taken from the suspension using a pipet were representative of the actual distribution but it did increase the likelihood that the results were more representative of the whole distribution. The suspension was then removed from the container using a pipet. A few drops were placed on a microscope slide and a cover slip was placed over them. Without the cover slide the microspheres clump together quickly making it very difficult for the program to separate the microspheres during the analysis. 
The statistical analysis provided the minimum, maximum, and mean diameter, count, skewness, and the 10, 25, 50, 75, and 90 percentiles based on both the number of microspheres and volume of microspheres for each microsphere series. The count is the number of microspheres used in the analysis and the skewness describes if the major fraction of the distribution is to the left or right of the center of the distribution. Higher values of skewness mean the distribution is left skewed and lower values mean the distribution is right skewed. Table 1 illustrates typical data obtained from these analyses. Tables providing the statistical analysis for each type of microsphere are in Appendix B. These tables were used to create bar graphs to graphically show the variation in diameter. Bar graphs were created by separating the data into increments of five microns. A typical bar graph is shown in Figure 11 with the bar graphs for the rest of the analyses in Appendix C. In these graphs the $\mathrm{x}$-axis represents the microsphere diameter range and the $\mathrm{y}$-axis represents the percentage of microspheres in a specific diameter range.

Table 1: S60HS Combined Table Showing both Numeric and Volumetric Distribution Data

\begin{tabular}{|c|c|c|}
\hline Description & $\begin{array}{c}\text { Numeric } \\
\text { Distribution }\end{array}$ & $\begin{array}{c}\text { Volumetric } \\
\text { Distribution }\end{array}$ \\
\hline Count & 751 & 751 \\
\hline $\begin{array}{c}\text { Microsphere } \\
\text { Diameter }\end{array}$ & $\mu \mathrm{m}$ & $\mu \mathrm{m}$ \\
\hline Minimum & 5.47 & - \\
\hline Maximum & 58.73 & - \\
\hline Mean & 16.42 & - \\
\hline 25-Quartile & 10.36 & - \\
\hline $\begin{array}{c}\text { 50-Quartile } \\
\text { (Median) }\end{array}$ & 14.00 & 30.00 \\
\hline 75-Quartile & 19.89 & - \\
\hline 10-Percentile & 8.61 & 16.20 \\
\hline 90-Percentile & 27.98 & 44.30 \\
\hline Skewness & 1.47 & - \\
\hline
\end{tabular}




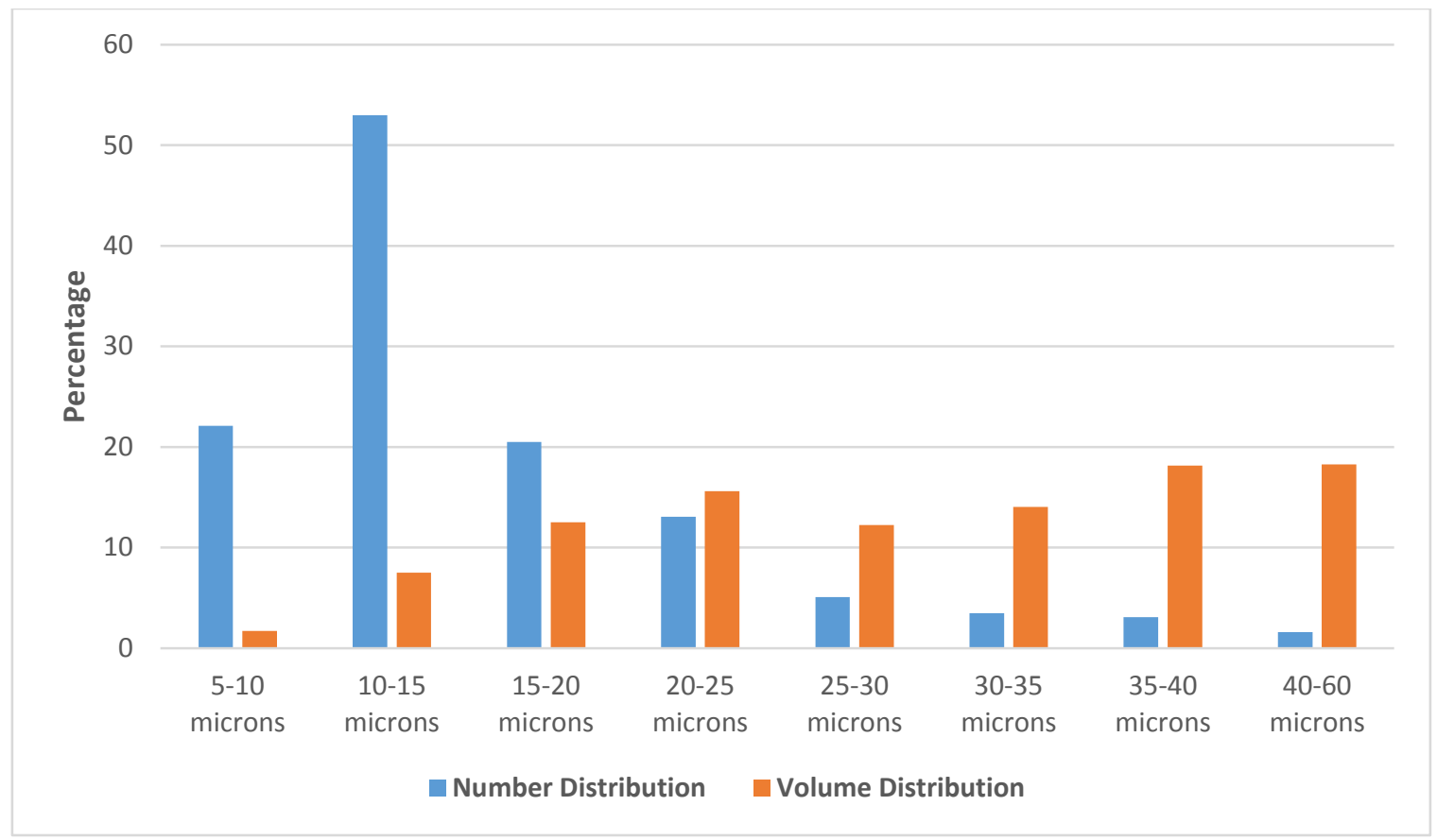

Figure 11: Number and Volume Microsphere Size Distribution for 3M's S60HS Microspheres Based on Microscope Analysis

\subsection{Microsphere Size Analyses Results}

The results for each of the optical microscopy and laser scattering analyses are provided in Appendices A through C. Appendix A explains the results of the laser scattering analyses for each of the microsphere series. Appendix B provides the numeric and volumetric distributions from optical microscopy results and compares ARC's results to 3M's provided data for volumetric distribution. Appendix $\mathrm{C}$ combines the numeric and volumetric distribution results from the microscopy analyses into bar graphs that highlight the differences between the two types of distributions. Table 2 combined the volumetric distribution results discussed in the appendices to make it easier to compare the results from all analyses. With the exception of the $10 \%$ volume distribution for the laser scattering analyses, all distribution results based on volume are within 10 microns of each other indicating the light scattering and microscopy counting produced similar results. This would indicate that both methods provide analyses that are 
accurate. As mentioned in Appendix A, the debris on the microspheres seems to have created a section of the distribution in the submicron range. This part of the distribution is bringing down the initial $10 \%$ value but seems to have corrected itself at the $50 \%$ and $90 \%$ where the volume of the larger microspheres has dwarfed the volume of the debris. Table 2 also provides the volumetric median, mean, mode and standard deviation from the laser scattering analyses. These properties were not available for the ARC microscopy analyses or the $3 \mathrm{M}$ product information as indicated by the dashes in the table. Results shown in Table 2 were calculated using computer software that provided results to four or more decimal places. It is questionable if the results are accurate to that many decimal places and are presented with values rounded to two decimal places instead.

There are advantages and disadvantages to each technique used in this research. Light scattering provides a quick, easy method for nondestructively measuring a distribution. Its downside is that the entire distribution is based on a limited number of data points calculated from the different angles used by the instrument. Results may be questionable if the optical properties of the solvent and particles are not accurately known or mixtures of particles with different compositions are present. Particles that absorb a large amount of the light may be problematic as they could fail to produce a usable scattering signal. Microscopy counting should produce results that are just as accurate as light scattering. This technique is not viable if time is a factor as it takes a long time to complete an analysis. The total number of particles used can also be an issue as smaller distributions lead to less accurate results. If particles are close together, it is unlikely that one will be able to determine if they are touching or actually one large particle. Depth of field will also be a problem if there are large differences in the size of particles. This is due to the fact that particles with large size differences cannot be in focus at the 
same time. The fact that both optical microscopy counting and light scattering produced similar results indicates that the error in both analyses is minimal [19], [23].

Table 2: Combined Volume Distribution Table for Microsphere Size Distribution. OM represents the ARC optical microscopy analyses. LS represents the light scattering analyses. PI represents data taken from $3 \mathrm{M}$ product information [24].

\begin{tabular}{|c|c|c|c|c|c|c|c|}
\hline $\begin{array}{c}\text { Microsphere } \\
\text { Type }\end{array}$ & Median & Mean & Mode & $\begin{array}{l}\text { Standard } \\
\text { Deviation }\end{array}$ & $10 \%$ & $50 \%$ & $90 \%$ \\
\hline $\begin{array}{l}\text { ARC HGM } \\
(\mathrm{OM})\end{array}$ & - & - & - & - & $40 \mu \mathrm{m}$ & $70 \mu \mathrm{m}$ & $103 \mu \mathrm{m}$ \\
\hline $\begin{array}{l}\text { ARC HGM } \\
\text { (LS) }\end{array}$ & $63 \mu \mathrm{m}$ & $63 \mu \mathrm{m}$ & $72 \mu \mathrm{m}$ & $26 \mu \mathrm{m}$ & $32 \mu \mathrm{m}$ & $63 \mu \mathrm{m}$ & $96 \mu \mathrm{m}$ \\
\hline $\begin{array}{c}\text { 3M S38HS } \\
\text { (PI) }\end{array}$ & - & - & - & - & $15 \mu \mathrm{m}$ & $40 \mu \mathrm{m}$ & $75 \mu \mathrm{m}$ \\
\hline $\begin{array}{c}\text { 3M S38HS } \\
(\mathrm{OM})\end{array}$ & - & - & - & - & $18 \mu \mathrm{m}$ & $53 \mu \mathrm{m}$ & $61 \mu \mathrm{m}$ \\
\hline $\begin{array}{c}\text { 3M S38HS } \\
\text { (LS) }\end{array}$ & $41 \mu \mathrm{m}$ & $43 \mu \mathrm{m}$ & $48 \mu \mathrm{m}$ & $26 \mu \mathrm{m}$ & $14 \mu \mathrm{m}$ & $41 \mu \mathrm{m}$ & $76 \mu \mathrm{m}$ \\
\hline $\begin{array}{c}\text { 3M S60HS } \\
\text { (PI) }\end{array}$ & - & - & - & - & $11 \mu \mathrm{m}$ & $30 \mu \mathrm{m}$ & $50 \mu \mathrm{m}$ \\
\hline $\begin{array}{c}\text { 3M S60HS } \\
(\mathrm{OM})\end{array}$ & - & - & - & - & $16 \mu \mathrm{m}$ & $30 \mu \mathrm{m}$ & $44 \mu \mathrm{m}$ \\
\hline $\begin{array}{l}\text { 3M S60HS } \\
\text { (LS) }\end{array}$ & $25 \mu \mathrm{m}$ & $24 \mu \mathrm{m}$ & $28 \mu \mathrm{m}$ & $13 \mu \mathrm{m}$ & $0.5 \mu \mathrm{m}$ & $25 \mu \mathrm{m}$ & $39 \mu \mathrm{m}$ \\
\hline $\begin{array}{l}\text { 3M IM16K } \\
\text { (PI) }\end{array}$ & - & - & - & - & $12 \mu \mathrm{m}$ & $20 \mu \mathrm{m}$ & $30 \mu \mathrm{m}$ \\
\hline $\begin{array}{c}\text { 3M IM16K } \\
(\mathrm{OM})\end{array}$ & - & - & - & - & $13 \mu \mathrm{m}$ & $20 \mu \mathrm{m}$ & $29 \mu \mathrm{m}$ \\
\hline $\begin{array}{l}\text { 3M IM16K } \\
\text { (LS) }\end{array}$ & $20 \mu \mathrm{m}$ & $19 \mu \mathrm{m}$ & $21 \mu \mathrm{m}$ & $9 \mu \mathrm{m}$ & $0.5 \mu \mathrm{m}$ & $20 \mu \mathrm{m}$ & $29 \mu \mathrm{m}$ \\
\hline $\begin{array}{c}\text { 3M IM30K } \\
\text { (PI) }\end{array}$ & - & - & - & - & $9 \mu \mathrm{m}$ & $16 \mu \mathrm{m}$ & $25 \mu \mathrm{m}$ \\
\hline $\begin{array}{c}\text { 3M IM30K } \\
\quad(\mathrm{OM})\end{array}$ & - & - & - & - & $12 \mu \mathrm{m}$ & $21 \mu \mathrm{m}$ & $31 \mu \mathrm{m}$ \\
\hline $\begin{array}{l}\text { 3M IM30K } \\
\text { (LS) }\end{array}$ & $18 \mu \mathrm{m}$ & $17 \mu \mathrm{m}$ & $21 \mu \mathrm{m}$ & $10 \mu \mathrm{m}$ & $0.4 \mu \mathrm{m}$ & $19 \mu \mathrm{m}$ & $28 \mu \mathrm{m}$ \\
\hline
\end{tabular}




\section{Ch. 4 Analysis of Strength}

\section{1: Types of strength Testing}

Since PWHGMs are a recent development, knowledge of their strength is minimal. Microspheres are difficult to test due to their size and shape. This presents multiple challenges in accurately testing the microspheres and interpreting the results. In current applications the failure strength of the microspheres is an important property to understand. Strength can be tested as a function of a large group of microspheres or for an individual microsphere. Some companies, like $3 \mathrm{M}$, provide a crush strength value that is calculated through testing a large volume of microspheres at once.

The crush strength provided by $3 \mathrm{M}$ is the isostatic crush strength of a large volume of microspheres. To calculate the isostatic compression of their microspheres, $3 \mathrm{M}$ uses a modified version of ASTM standard D 3102-78: Standard Practice for Determination of Isostatic Collapse Strength of Hollow Glass Microspheres. The standard was developed in 1978 and involves crushing large volume of microspheres at once instead of examining the properties of individual microspheres. Six years later, in 1984, the standard was withdrawn with no replacement for unspecified reasons. In the following paragraph the ASTM standard is explained and the differences between 3M's method and the standard are detailed [25].

For this test, microspheres with a volume of 3 to $6 \mathrm{~cm}^{3}$ are sealed in a rubber balloon with some excess glycerin or isopropyl alcohol. The balloon is then placed into a pressure chamber filled with hydraulic oil. As pressure is applied, the changes in volume and pressure are recorded as electrical output received from sensors on an $x-y$ recorder. This data is used to calculate what percentage of the microspheres has collapsed. The crush strength is recorded as 
the pressure that caused a predetermined percentage of microspheres to fail. The following paragraphs describe the test procedure in greater detail [25], [26].

First, the sensors reading the changes in pressure and volume are calibrated for testing so the recorder receives data from the sensors as $1.00 \mathrm{ml} / \mathrm{mV}$ and $1.38 \mathrm{MPa} / \mathrm{mV}$. Next, the initial volume of the glass is calculated using the true density of the microsphere glass and the weight of the microspheres in the balloon using Equation (1) [25], [26].

$$
V_{g}=\frac{M}{\rho}
$$

In Equation (1), $V_{g}$ is the true glass volume, $M$ is the mass of the microspheres, and $\rho$ is the true density of the glass. Before testing, the balloon is weighed using a scale and air comparison pycnometry. The balloon is then filled with microspheres and the process is repeated to calculate the mass of the microspheres themselves. The microsphere volume $\left(V_{b}\right)$ is found by using the pycnometry data and the void volume $\left(V_{v}\right)$ calculated using the glass and bubble volume through Equation (2) [25], [26]:

$$
V_{v}=V_{b}-V_{g}
$$

After the mass of the microspheres has been calculated, alcohol is added and the balloon is sealed once all air has been removed. Once the balloon with the microspheres is ready, it is inserted into the pressure chamber and the chamber is filled with oil. Pressure is increased in the chamber and the results are recorded as the collapse curve. Next pressure in the chamber is removed and a 20 minute wait period is taken to allow the oil to reach its initial thermodynamic state. Pressure is then reapplied to record the compression curve. Figure 12 illustrates the typical relationship between applied pressure and volume. Total volume collapse $\left(V_{T}\right)$ is 
calculated by subtracting the collapse curve volume from the compression curve volume at zero pressure. The void volume collapse $\left(V_{p}\right)$ at any pressure is calculated via Equation (3) [25], [26]:

$$
V_{p}=V_{T}-\left(V_{\text {compression }}-V_{\text {collapse }}\right)
$$

$V_{\text {compression }}$ is the volume of the compression curve at a selected pressure and $V_{\text {collapse }}$ is the volume of the collapse curve at the same pressure. The volume collapse fraction is calculated by dividing $V_{p}$ by $V_{v}$ as seen in Equation (4). Crush strength is recorded as the pressure that causes the volume collapse fraction to hit a predetermined value [25], [26].

$$
V_{\text {Collapse Fraction }}=\frac{V_{P}}{V_{V}}
$$

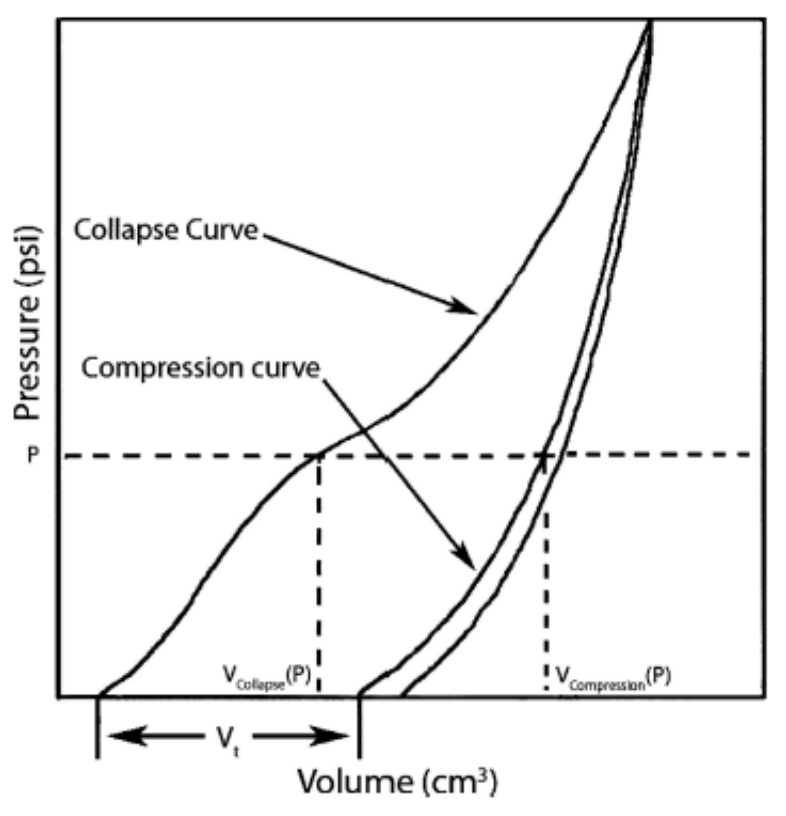

Figure 12: Microsphere Breakage Curve [26]

For 3M's variation (3M QCM 14.1.5), testing under 6000 psi is done pneumatically while testing above 6000 psi is hydraulic. 3M uses a cutoff of $10 \%$ failure for their crush strength. This means the crush strength $3 \mathrm{M}$ records is the pressure that occurs at a $V_{\text {Collapse Fraction }}$ of 0.1 . 
Engineers at $3 \mathrm{M}$ emphasized that this test is mainly for quality control and is not meant to be used to design applications [25], [26].

There are multiple types of stress testing that could be performed on individual microspheres, most of which do not result in uniform stress. These methods include tensile testing, flexural testing, and compressive testing. Each of these tests determines the type of stress at which a glass will fail for the type of loading indicated in the test. In the following sections a description of each type of test is provided and the practicability of each test is discussed.

In tensile testing force is applied uniaxially, stressing the specimen until it fails as seen in Figure 13. The test specimens for a material usually take the form of a straight bar or rod with a uniform cross section. Tensile stress, $\sigma$, is calculated by taking the quotient of the load at failure, $F$, over the cross sectional area, $A$, as shown in Equation (5).

$$
\sigma=\frac{F}{A}
$$

With tensile testing, it is difficult to test even simple shapes of glass, let alone microspheres. Any additional types of stress created during testing may affect the results of the test [27], [28].

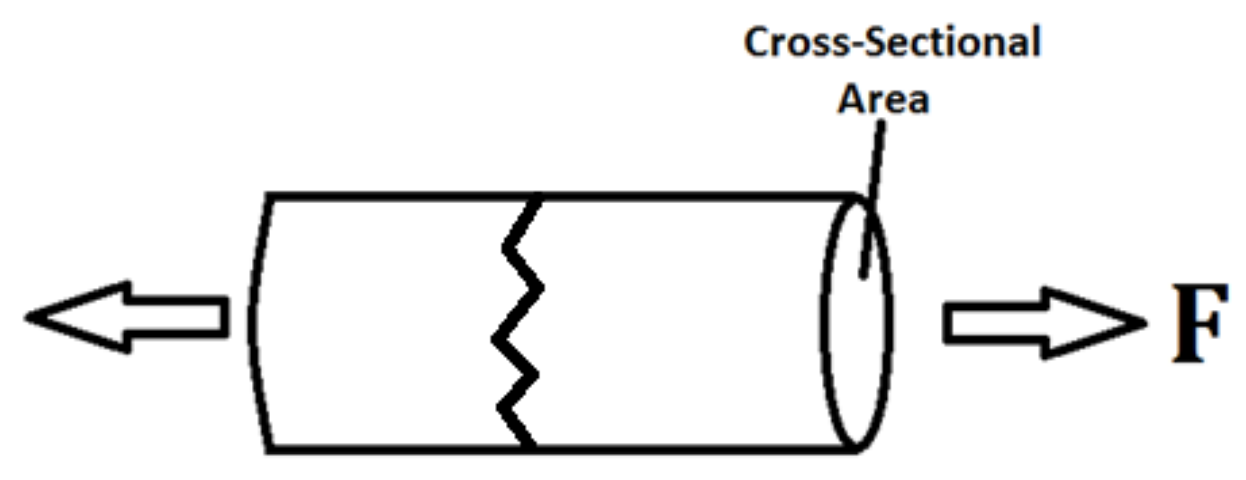

Figure 13: Uniaxial Tension Test 
One unique tensile test method was developed by Los Alamos National Labs in the 1970s. In their test, an individual microsphere was separated and bonded to two small brass rods at opposite ends of the microsphere using epoxy. Care was taken to prevent epoxy from bonding the glass rods together. A special apparatus was developed to pull the rods apart and record data [25]. This type of test would not be practical for measuring strengths for the large number of microspheres needed to acquire confidence in the results.

Flexural tests, also known as bending tests, take samples of uniform length with a square, rectangular, or circular cross section and hold them in place using supports set at a predetermined length apart. Force is applied to the top of the sample via one or two contact points to cause bending. The applied force is continuously increased until failure occurs in the sample. As seen in Figure 14, a three point bend test applies the load directly to the center of the sample [27].

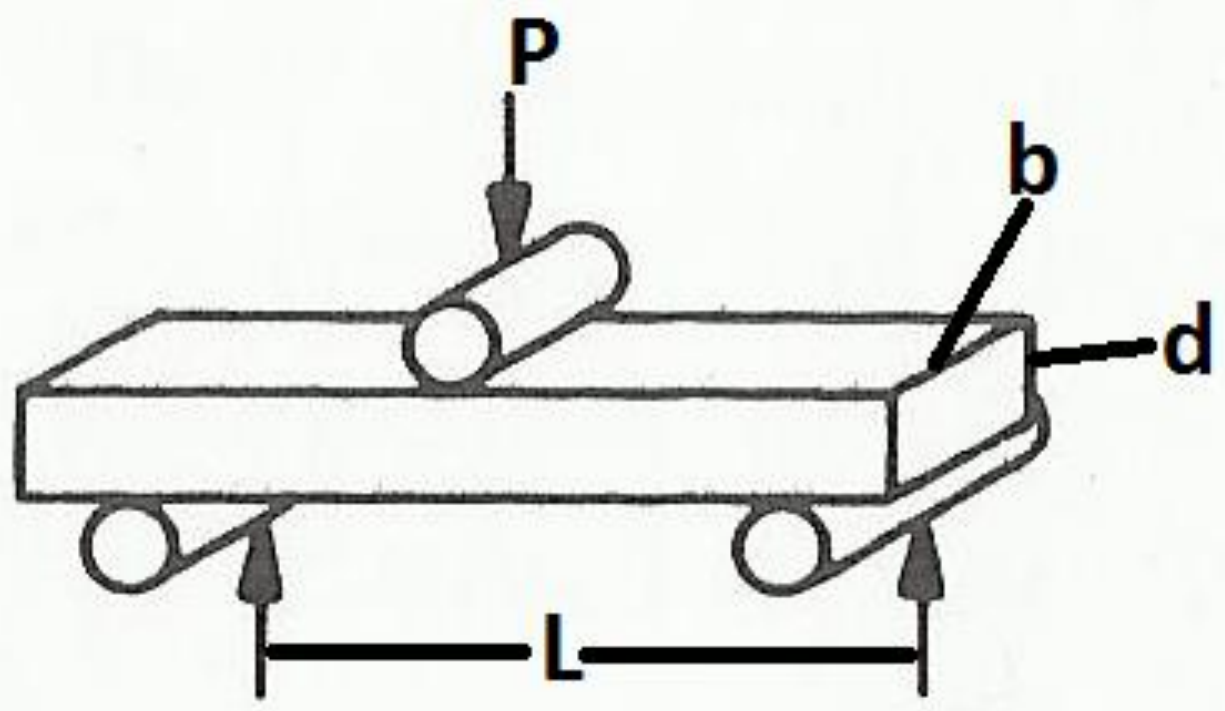

Figure 14: 3-pt Bend Test [27] 
Strength calculated from the test is recorded as the modulus of rupture, $S$. Modulus of Rupture is calculated using Equation (6) for three point bend testing.

$$
S=\frac{3 P L}{2 b d^{2}}
$$

In the equation, $P$ is the force applied, $L$ is the length between the supports, $b$ is the width of the sample, and $d$ is the height of the sample [27]. Since this is a non-uniform loading test, for the equation to be valid, failure of the sample must occur at the midsection of the bottom surface.

For hollow microspheres, a pseudo three point bend test was created by Bratt et al. Bratt et al used the ASTM three point bend test to develop a theory on how uniaxial forces act on a hollow microsphere. In the theory, the sphere will experience compressive force around the vertical axis of the sphere and tensile force around the center of the sphere as seen in Figure 15. Another aspect of the theory was that failure did not occur at the contact point, but around the equator of the sphere due to tensile forces. When the force at the equator exceeded the tensile strength of the material, fracture would occur as a circumferential crack around the equator. The theory used uniaxial compression to apply force at the contact point and treated the equator of the microsphere as the test supports, modifying Equation (6) into Equation (7) [29] [30]:

$$
\sigma=\frac{3 P L}{2 b d^{2}}=\frac{3 F\left(\frac{d}{3}\right)}{2 \pi d t^{2}}=\frac{F}{2 \pi t^{2}}
$$

In Equation (7), $F$ is the uniaxial force, $d$ is the diameter of the microsphere, and $t$ is the wall thickness. Plates are used to apply force on the spheres. Bratt et al's theory simplifies the types of stresses acting on the sphere and does not take into account diameter when calculating stress. Using this test method and Equation (7), Bratt et al obtained strength values ranging from 
1.2 to $4.3 \times 10^{5}$ psi on unspecified microsphere compositions. The range of wall thicknesses used was 0.77 to 2.4 microns [29].

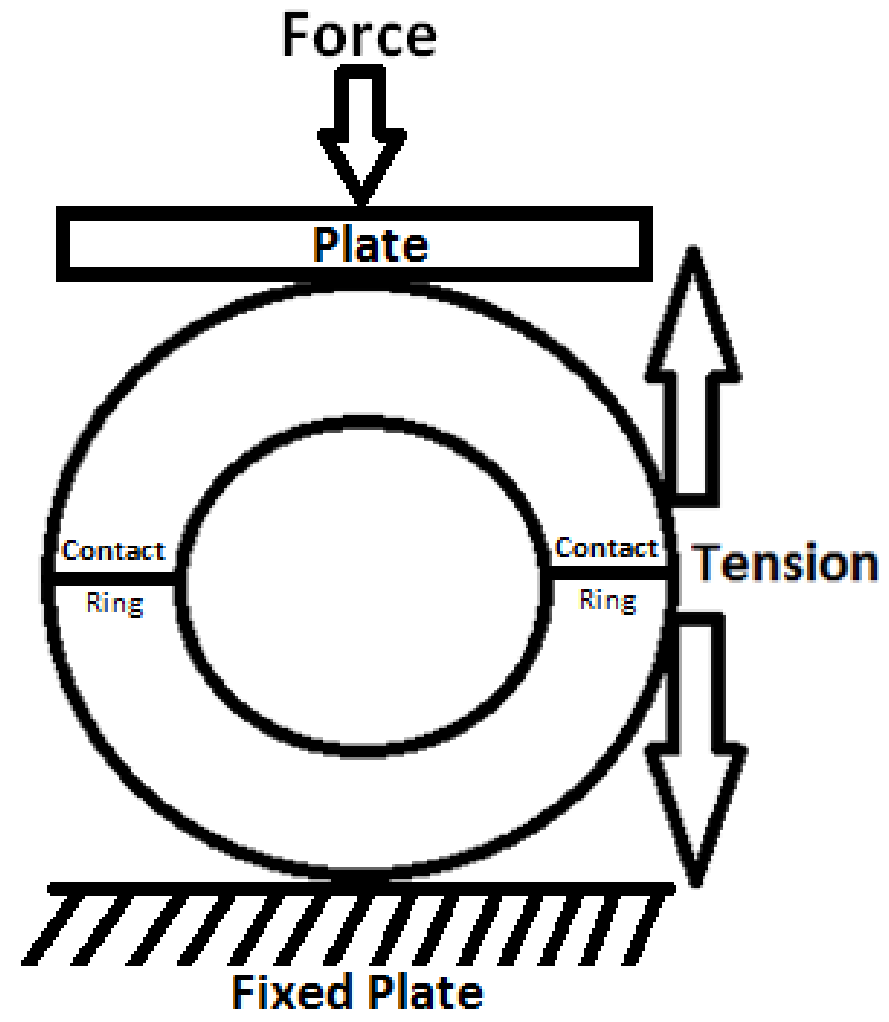

Figure 15: Two Dimensional Representation of a Thin Walled Microsphere Illustrating Proposed Stresses Based on Theory by Bratt et al [29]

Compression testing is used to determine the stress at failure when a glass is crushed. The method of testing is similar to tensile testing except that force is applied to crush the material instead of pulling it apart. Most tests use a simple shape as the test specimen, such as a straight bar or rod, and place it between two plates. Compressive force is applied to one or both plates to crush the sample as seen in Figure 16. Strength is calculated by taking the quotient of the force applied over the cross sectional area of the sample and uses Equation (5) like tensile testing [27]. This test can be adapted to microspheres using a nanoindenter. Force would be applied to the microspheres via contact loads, initially at one point, on the top and bottom of the 
sphere. This method of stress testing is probably the most practical for microspheres out of all the tests discussed.

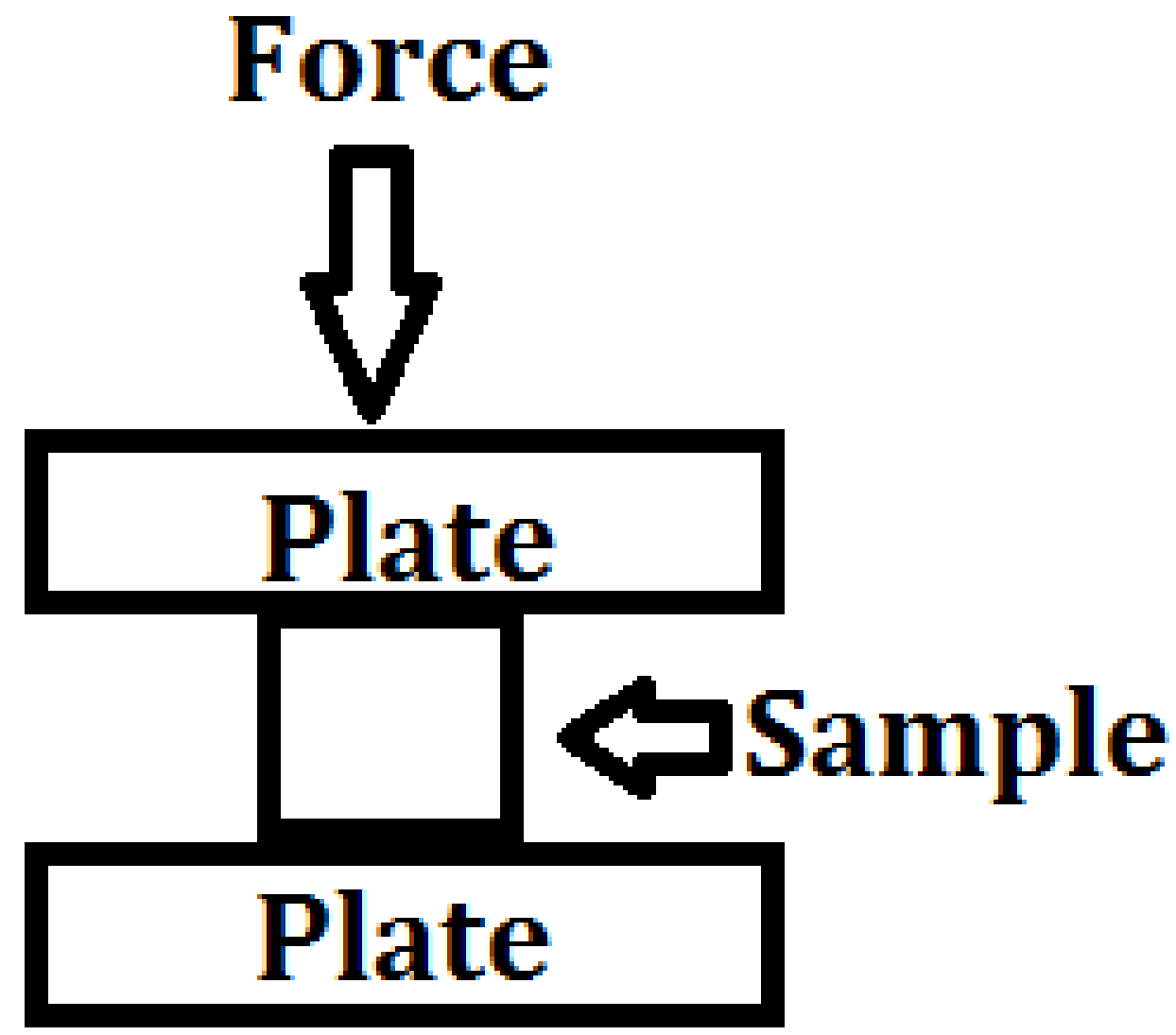

Figure 16: Typical Compression Test

\section{2: Nanoindentation of Microspheres}

Presently, the most extensive published research on the compressive properties of individual microspheres is from the University of Alabama, Birmingham with help from the Los Alamos National Laboratory [25]. The research was published over a series of papers, each one either expanding on their interpretation of the results or explaining the results for different types of microspheres. In the initial research, Koopman et al tested HGMs from 3M with diameters between 5 and 90 microns and wall thicknesses between less than one micron and two microns. 
These microspheres were sprinkled onto a polished aluminum substrate and crushed using an 89 micron, flat-end cylindrical tip made of sapphire. The tests were run on a MTS-Nano Instruments XP-II nanoindenter at a strain rate of $0.05 / \mathrm{s}$ and with no limit on the tip displacement [31].

Tests results were presented as tip displacement vs load applied as shown in Figure 17. In Figure $17 \mathrm{MB}$ is the acronym for microballoon, another term used for microspheres. Koopman et al presented results as the fracture load and fracture energy vs microsphere diameter. Fracture energy was the area under the curve up to the point of failure on the load vs displacement graph and was calculated mathematically using Equation (8) [31]:

$$
W_{f}=0.5 P_{f} \delta_{f}
$$

In Equation (8) $P_{f}$ is the fracture load and $\delta_{f}$ is the tip displacement at failure. Koopman et al used their initial paper to prove that it was possible to analyze the mechanical properties of a microsphere with nanoindentation [31].

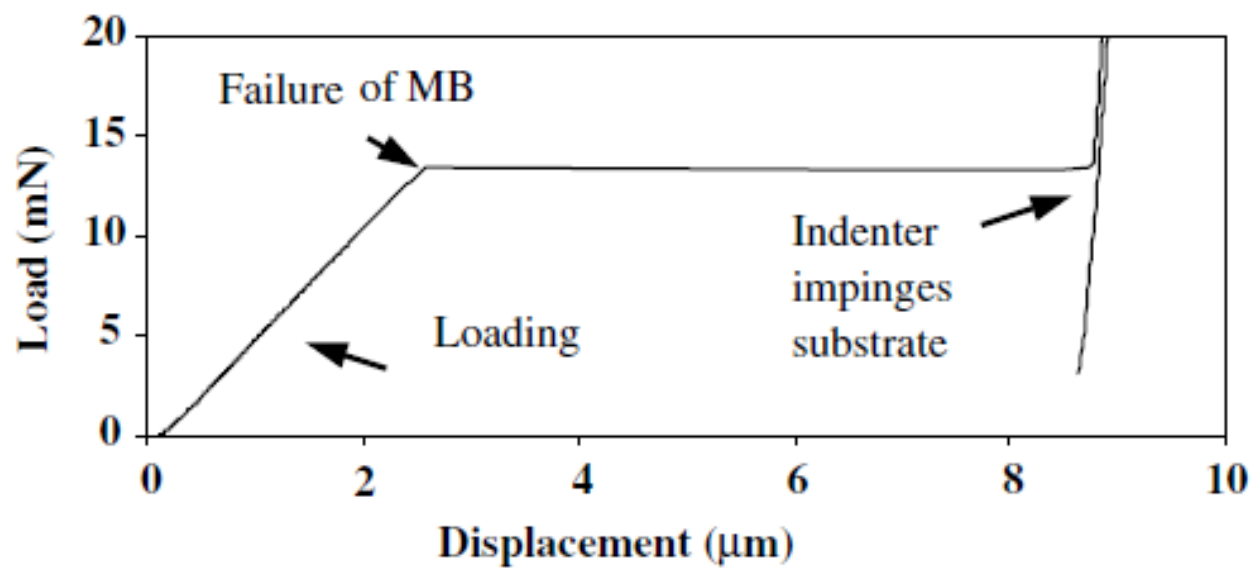

Figure 17: Nano Crush Test Results for Koopman Et Al [31] 
In their next two papers, Carlisle et al describe in more detail the methods used to analyze their data. After examining HGMs, the group worked on different types of hollow carbon microspheres (HCM). In addition to basic HCMs, the group tested HCMs with various types of coatings. Tap density was used as a way to separate out a group of microspheres based on their wall thickness as the group did not have a better way of measuring the wall thickness of each microsphere tested. Three different types of results were observed in the nanoindenter data. These different results were labeled single walled, flawed single walled, and nested as seen in Figure 18.

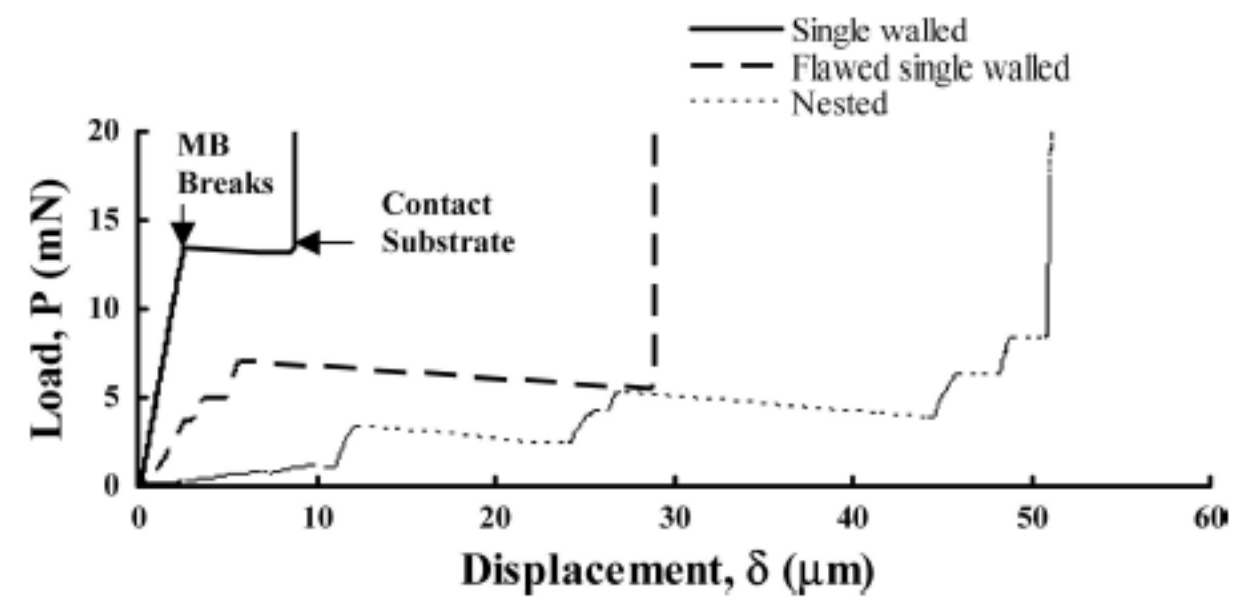

Figure 18: Different Types of Nano Crush Test Results [25],

Different results were thought to be caused by morphology variations in the microspheres. The single walled result was the result seen in normal microspheres. Flawed single walled results were thought to be caused by irregularities or flaws in the surface and sphere wall. Nested results were thought to be caused by several internal compartments within the microsphere [25], [32], [33], [34]: 


\section{3: Fracture of Glass Microspheres}

Glasses are more prone to brittle failure than metals or polymers due to their bonding and structure. Flaws are a major reason that glasses exhibit relatively low as well as a wide range of measured strengths. Brittle failure occurs in a material when there is little to no plastic deformation before failure [27], [35]. Glasses are amorphous materials and do not plastically deform under stress. This means stresses can be concentrated in the presence of flaws resulting in brittle fracture. As described below, stress concentrations at flaw tips result in low measured strengths [10], [35].

The current understanding of how brittle fracture occurs in materials was developed by Alan Griffith in 1921 [36]. Griffith was trying to understand why materials failed at stresses lower than theoretically predicted. Griffith used Charles Inglis's earlier work which examined the stress concentration at the tip of a crack in a brittle material and determined that the maximum stress at a crack tip is equivalent to Equation (9) [10], [28]:

$$
\sigma_{m}=2 \sigma_{a}\left(\frac{c}{\rho}\right)^{0.5}
$$

Equation (9) shows that the maximum stress at a crack tip, $\sigma_{m}$, depends on the applied stress, $\sigma_{a}$, the length of the crack, $c$, and the radius of the crack tip (which is usually assumed to be on the order of the spacing of atoms), $\rho$. Applying data representing crack measurements to Equation (9) show the stress at the crack tip is much larger than the applied stress and that longer cracks result in smaller $\sigma_{a}$ values required to yield the same $\sigma_{m}$. Failure occurs when $\sigma_{m}$ is equal to the theoretical strength of the glass $\left(1.4-4.2 \times 10^{6} \mathrm{psi}\right)$ at the crack tip [37]. Under uniform stress conditions the largest crack in a system is the one that will lead to failure. Inglis's showed 
that flaws like cracks and pores, even small ones, create large stress concentrations which lead to failure at lower applied stresses than theoretically predicted [28].

Griffith continued Inglis's work to develop the theoretical basis of fracture mechanics for brittle materials. In the theory, the propagation of a crack causes a decrease in the stored elastic energy of the material while causing an increase in surface energy because the crack creates a new surface when it propagates. The crack length will only increase if the decrease in internal energy exceeds the increase in surface energy. Griffith used the first law of thermodynamics to derive the fracture stress of an elliptical crack in a flat plate. Griffith assumed heat is zero for the amount of time necessary for a crack to propagate, related work to internal energy, and made internal energy the combination of the surface energy increase and elastic energy decrease caused by crack propagation. Inputting the equations for surface energy and elastic energy will result in Equation (10) for fracture [28].

$$
\sigma_{f}=\left(\frac{2 E \gamma}{c}\right)^{0.5}
$$

A generalized form of Equation (10) is Equation (11):

$$
\sigma_{f}=A\left(\frac{E \gamma}{c}\right)^{0.5}
$$

In Equation (11), $A$ represents a constant that changes via sample and crack geometry, $E$ represents the Elastic modulus, and $y$ represents the surface energy. This generalized form for fracture stress is used today to estimate the strength of brittle materials. All glasses, including glass microspheres, have flaws due either to manufacturing or subsequent use that leads to lower strengths than theoretically predicted. Equation (11) is consistent with Equation (9) in that the measured strength required to fracture a glass is inversely related to the size of the flaw, c. As 
previously stated, under uniform conditions of stress the largest flaw in the microsphere will lead to failure. However under non uniform stress conditions, the critical flaw that leads to failure may not be the largest in the microsphere but will be the flaw where stress at the crack tip is equal to the material's theoretical strength as shown in Equation (11). This concept is very important since most loading conditions for microspheres do not result in uniform stress [10].

\section{4: Weibull Analysis of Strength Data}

One conclusion drawn from Griffith's work (Equation (11)) for statistical analysis is that the fracture strength for a group of samples made from the same material will be different based on the different flaw sizes present in each sample. This variation in the flaw sizes leads to the scattering of strength data. If failure occurs at any flaw in a sample, it will eventually lead to total failure of the sample. According to Equation (11), a specimen will fail at the largest flaw size when the stress is uniform throughout. This conclusion is known as the weakest link theory. As a consequence, strength data are skewed to larger values and typical Gaussian statistics do not present an accurate representation of the data [10], [28], [38], [39].

The Weibull distribution is a probabilistic analysis created by Waloddi Weibull in 1939 [38]. It is different from other distributions, such as Gaussian, because it takes into account the effect that sample volume has on strength and is an extreme value distribution. Samples of varying volumes will have different quantities of flaws with larger volumes having a larger number of flaws. The Weibull analysis plots the results of multiple tests and extrapolates a line from the data, known as the Weibull equation, which represents the probability that a material will fail at a given stress. Data sets with a larger number of results provide a more reliable experimentally derived Weibull equation. The analysis can be more useful than using an average 
value for strength since different sized flaws in a brittle material will cause the material to fail at different strength values.

Weibull analysis is also able to estimate how the change in volume will affect the strength of a material. Along with using the weakest link theory, the Weibull analysis assumes that any unit volume will have roughly the same number of flaws, though the flaw size distribution between samples does not have to be the same as seen in Figure 19. The Weibull analysis also assumes that the probability of failure due to a given stress is directly related to the number of flaws present. In other words, the probability of finding a critical flaw for an applied stress is directly related to the volume under that stress. Referring to Figure 19, there is a $100 \%$ probability that the large sample will fail at the circled crack at a stress determined by Equation (11). On the other hand there is a $10 \%$ probability that failure will occur at that same stress in the set of smaller samples.

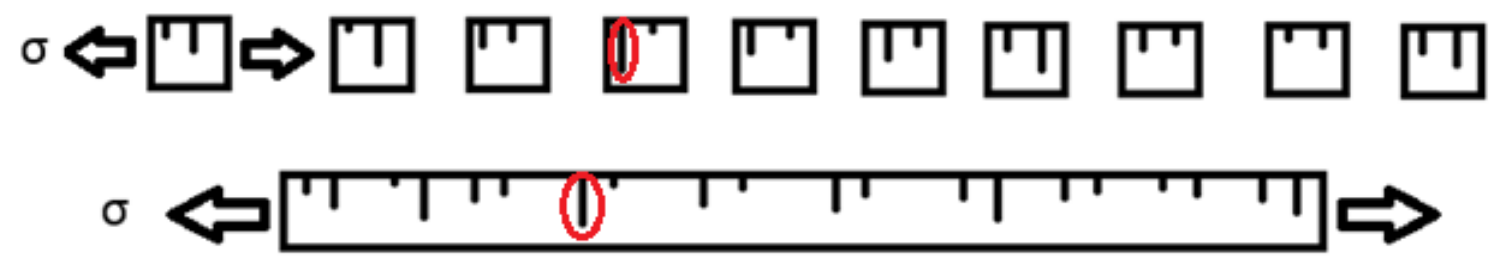

Figure 19: Flaw Size and Number Distribution between Different Samples

After analyzing one set of samples at a particular volume, the analysis can be scaled to predict when samples at another volume might fail. This lowers the number of samples that need to be tested in order to determine the probability of failure at different volumes. There are also ways to modify different data sets so they can be combined to form one large data set [10], [28], [38], [39], [40]. 
The Weibull distribution is commonly expressed as a cumulative distribution function (CDF). In a $\mathrm{CDF}, \mathrm{F}(\mathrm{x})$ is the set of data with values of $X \leq x$ divided by the total number of samples. $F(x)$ represents the probability that a random value, $X$, from the data set will have a value less than or equal to $x$. When used to represent failure stresses, $F(x)$ is the probability of failure, $P_{f}$, and $x$ is the stress resulting in failure, $\sigma$. The equation for a CDF is expressed as Equation (12) [39], [41]:

$$
P_{f}(X \leq \sigma)=F(x)=1-e^{-V \gamma(\sigma)}
$$

In Equation (12), $V$ is the sample volume and $\gamma(\sigma)$ is a representation function that Weibull required to have two properties. One, that it be a positive nondecreasing function, meaning that as $\sigma$ increases the output of the function must also increase. Two, the function becomes zero when $\sigma$ is equal to or less than the value of the parameter $\sigma_{\mu}$, the smallest stress at which a specimen can fail. Weibull determined the simplest function that meets the requirements was Equation (13) [39], [41]:

$$
\gamma(\sigma)=\frac{1}{V_{0}}\left(\frac{\sigma-\sigma_{\mu}}{\sigma_{0}}\right)^{m}
$$

Substituting Equation (13) into $\gamma(\sigma)$, changes Equation (12) to Equation (14)

$$
P_{f}=1-e^{-\frac{V}{V_{0}}\left(\frac{\sigma-\sigma_{\mu}}{\sigma_{0}}\right)^{m}}
$$

Equation (14) is known as a three parameter Weibull analysis. In the equation $V_{0}$ is a chosen unit volume that normalizes the volume variable and $m, \sigma_{0}$, and $\sigma_{\mu}$ are statistical parameters that represent different aspects of the equation. $V / V_{0}$ is used to transform a Weibull analysis to different volumes, allowing one to predict how failure will occur at other volumes and reduce the amount of testing required to understand a material. When examining a data set 
where $V=V_{0}, V / V_{0}$ becomes unity. To determine the Weibull parameters, Equation (14) is expanded using logarithms as seen in Equation (15) [28], [42]:

$$
\operatorname{Ln} \operatorname{Ln}\left(\frac{1}{1-P_{f}}\right)=m \operatorname{Ln}\left(\sigma-\sigma_{\mu}\right)-m \operatorname{Ln}\left(\sigma_{0}\right)+\operatorname{Ln}\left(\frac{V}{V_{0}}\right)
$$

When $\operatorname{Ln} \operatorname{Ln}\left(\frac{1}{1-P_{f}}\right)$ is plotted against $\operatorname{Ln}\left(\sigma-\sigma_{\mu}\right)$, Equation (15) appears as a straight line with a slope of $m$ and a y axis intercept of $-m \operatorname{Ln}\left(\sigma_{0}\right)+\operatorname{Ln}\left(\frac{V}{V_{0}}\right)$. When $V / V_{0}=1$ the intercept is $-m \operatorname{Ln}\left(\sigma_{0}\right)$

In Equation (15), $m$ is the slope and is called the Weibull modulus. In statistics, $m$ is known as the shape parameter and describes the general shape of curve based on how the data set is distributed. Lower values represent a right skewed distribution and higher values represent a left skewed distribution. Higher values of $m$ are desired as it indicates that variations in failure stress typically become smaller. This is desirable when designing materials, as it brings the fracture stress of a material closer to a more predictable, single value [28], [43], [44], [45].

In statistics $\sigma_{0}$ is known as the scale parameter which defines the range of the distribution. As $\sigma_{0}$ becomes larger, a material requires a higher stress to cause failure but the range over which failure can occur is also stretched. In the Weibull analysis, the characteristic stress represents the applied stress where $63.2 \%$ of samples will have failed. This probability of failure will always occur in an analysis when $\sigma-\sigma_{\mu}=\sigma_{0}$ [28], [43], [46], [47], [48].

$\sigma_{\mu}$ is the threshold parameter and represents the stress below which failure will not occur. Unlike the other Weibull parameters, $\sigma_{\mu}$ does not have to be calculated through the extrapolated linear Weibull equation. Instead it can be chosen based on a user defined parameter of the 
minimum stress at which failure will occur. This could be the lowest stress of the data set, the minimum stress to cause failure for a certain flaw size, or any other way the user sees fit to define it. $\sigma_{\mu}$ will also stretch out the stress data on the plot, changing the Weibull modulus and characteristic strength. The most common method is to set $\sigma_{\mu}$ to 0 and assume there is no stress below which failure will not occur. Setting $\sigma_{\mu}$ to 0 essentially removes it from the analysis reducing the number of parameters in the Weibull equation to two, $\mathrm{m}$ and $\sigma_{0}$, as shown in Equation (16) and illustrated in Figure 20 [28], [43]:

$$
\operatorname{Ln} \operatorname{Ln}\left(\frac{1}{1-P_{f}}\right)=m \operatorname{Ln}(\sigma)-m \operatorname{Ln}\left(\sigma_{0}\right)+\operatorname{Ln}\left(\frac{V}{V_{0}}\right)
$$

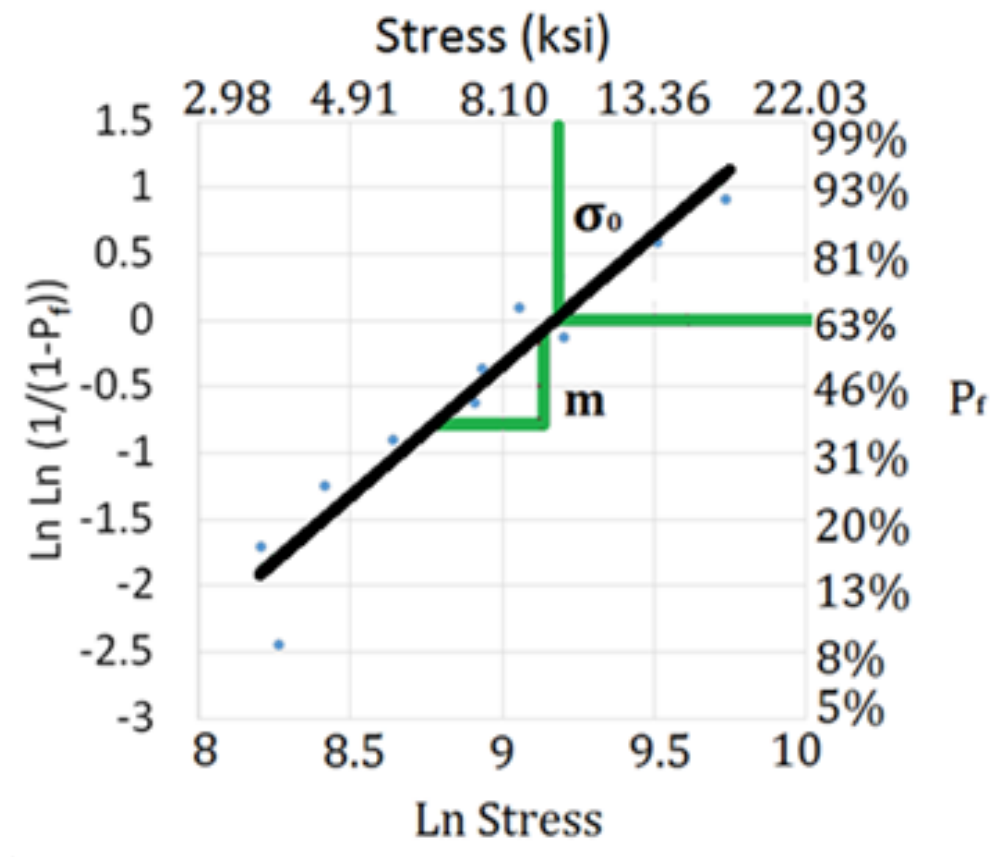

Figure 20: Two Parameter Weibull Plot Showing how the Weibull Parameters are Determined Graphically

Figure 20 shows that when the left side of Equation (16) is plotted against the natural log of applied stress a straight line is obtained. Both $m$ and $\sigma_{0}$ can be graphically derived from this 
plot. For convenience, the actual stress and probability of failure are also shown. The probability of failure for each point of data is calculated using Equation (17) [28], [42], [49]:

$$
P_{f}=\frac{n}{N+1}
$$

In Equation (17), $n$ is number of samples that have failed at or less than the current sample's stress and $N$ is the total number of samples tested. Data sets are usually rank ordered from lowest to highest stress where $n$ is the rank of a specific stress. Using $N+1$ in Equation (17) ensures $P_{f}$ is always less than 1 . Allowing $P_{f}=1$ would create an incalculable 1 divided by 0 situation when inserted into the Weibull equation [28], [42].

The equations used above represent the Weibull analysis for uniform uniaxial tension or compression [50]. To use the Weibull analysis for analyzing other types of stress distributions an additional parameter must be introduced. This parameter is known as the load factor, $k$, and is a function of the Weibull modulus and the test geometry calculated through Equation (18) [28], [40], [51]:

$$
k=\frac{1}{V} \int_{V}\left(\frac{\sigma}{\sigma_{0}}\right)^{m} d V
$$

In Equation (18), $V$ is the sample volume over which stress is applied. When this is the whole specimen as seen in uniform, uniaxial tension or compression, $k$ becomes unity. This is not the case with other stress modes, such as bending, where the stress varies as a function of position. With the addition of the load factor, Equation (14) will become Equation (19) [28], [40]: 


$$
P_{f}=1-e^{-k \frac{V}{V_{0}}\left(\frac{\sigma-\sigma_{\mu}}{\sigma_{0}}\right)^{m}}
$$

As mentioned earlier it is possible to combine multiple sets of data to create one large set. These sets do not need to have samples that are the same volume or that were tested using the same methods. Using this method it is possible to combine data from different strength tests or estimate how a material will act under different types of stress based on the results of one data set. Equation (20) is used to combine data sets by converting the probability of failure for each data point from a data set calculated using a specific volume or test method to the probability of failure for a different volume or test method [28], [40].

$$
\frac{\operatorname{Ln}\left(1-P_{F a}\right)}{\operatorname{Ln}\left(1-P_{F b}\right)}=\frac{-(k V)_{a}\left(\frac{\sigma-\sigma_{\mu}}{\sigma_{0}}\right)^{m}}{-(k V)_{b}\left(\frac{\sigma-\sigma_{\mu}}{\sigma_{0}}\right)^{m}}=\frac{(k V)_{a}}{(k V)_{b}}
$$

Another key aspect of the Weibull analysis is the number of samples tested. As with many forms of statistics, there is a confidence level for any analysis. In the Weibull analysis, this level of confidence in the accuracy of the data is determined by the number of samples in a data set. There are studies that have examined the $\%$ potential error in an analysis of $m$ based on the number of samples and shown that the relationship is logarithmic. The confidence level of 10 samples is $+/-40 \%$ while the confidence level for 60 samples is $+/-10 \%$. This is an important aspect to consider as a set of data may fit the Weibull equation well but actually contain significant error [27]. 


\section{Ch. 5: Experimental Design and Methods of Data Analysis}

\section{1: Crush Strength Testing}

As described earlier, crush testing was selected because it provides the valuable data for many conditions which microspheres may encounter. Data provided by microsphere manufacturers has focused on the bulk crush strength of the microspheres making it difficult to assess the strength of individual microspheres. Nanoindentation provides an alternative to bulk crush testing and was used to determine the strengths of individual microspheres in this study. Each microsphere was crushed between a flat end indenter tip and a substrate below the microsphere. Due to the statistical variation in the strength of glass, data sets were analyzed using the Weibull analysis to determine the scattering in strength and the characteristic failure stress of the microspheres. The analysis was used to show differences in strength for different types of microspheres and microspheres of different diameters.

\section{2: Experimental Parameters}

There are multiple physical and chemical characteristics that could potentially affect the

microsphere's strength. Various properties such as microsphere diameter, pore diameter, wall thickness, and chemical composition could potentially cause some variation in the microsphere's strength. For the experiments discussed in this thesis, microsphere diameter and wall thickness were chosen as the factors against which strength was tested and stress was calculated. These factors should directly relate to strength due to the relationship between volume and the number of flaws within that volume. To visibly show changes in strength, microspheres were separated into three diameter ranges. These ranges were about 10 microns wide and each range was 5 microns apart from the next range. Wall thickness was measured by examining broken microspheres using a SEM. This parameter was measured two ways. In the first method, tested 
microspheres were identified in the SEM and had their wall thickness measured. For the second method, multiple microspheres were broken and measured to correlate average wall thickness to microsphere diameter. This method was used when a tested microsphere could not be found and its wall thickness was unknown.

Five different types of microspheres, produced by both $3 \mathrm{M}$ and $\mathrm{ARC}$, were used in the crush strength tests described below. Three types of ARC microspheres from different stages of PWHGM production were tested. These microspheres include HGMs that were not heat treated or leached, heat treated and nonleached HGMs, and PWHGMs. Using microspheres from these different stages of production showed how the crush strength changes throughout the production of the PWHGMs. The last sets of microspheres were the S38HS and S60HS microspheres from 3M. These were used as a control group to compare PWHGMs to some of the microspheres available commercially.

For the experimental parameters detailed above, there were ten microsphere diameter ranges tested. Three diameter ranges were tested for both the initial ARC HGMs and the heat treated ARC HGMs, two for the PWHGMs, and one for the S38HS and S60HS 3M HGMs. The number of ranges was determined based on the microspheres provided and time available to complete testing. All microspheres used for experiments were provided by ARC. ARC HGMs were provided with a diameter range of approximately 25 microns to 75 microns. Heat Treated ARC HGMs were created by heat treating a small quantity of ARC HGMs at temperatures over $500^{\circ} \mathrm{C}$ for longer than 6 hours followed by air cooling. Because they were originally normal ARC HGMs, they have the same diameter range. Data collected from these microspheres was divided into ranges of 30-40 microns, 45-55 microns, and 60-70 microns. The PWHGMs were only available in the 25 to 50 micron range and were divided into 30-40 micron and 40-50 
micron sets. The S38HS microspheres were available in 10 to 95 micron range, but only the 8090 range was tested. Similar to the S38HS microspheres, the S60HS microspheres were available between 10 and 90 microns in diameter but only the 50-60 micron range was tested. For each diameter range 10-11 microspheres were tested, with the exception of the PWHGMs and the 45-55 micron range heat treated ARC HGMs. For these three diameter ranges 50 microspheres of each type were tested. The average time required to test a single microsphere, including sample preparation, was about 30 minutes.

\section{3: Sample Preparation}

Samples consisted of microspheres adhered to fused silica slides. Fused silica was used as it is harder than the porous amorphous silica found in the PWHGMs and the borosilicate glass of the other microspheres. Apeizon L high vacuum grease was chosen as the adhesive for two reasons. One, most adhesives harden and are weaker than the microspheres themselves, thus the adhesive would adsorb some of the force used to crush the microspheres, skewing the data. Grease on the other hand will adhere the microsphere to the substrate, but will be pushed out of the way until the microsphere has contact with just the substrate. Second, high vacuum grease is required for use in an SEM where the low pressures could cause many other adhesives to outgas, causing potential damage to the instrument.

Samples were made by spreading a small amount of grease onto the fused silica substrate as thinly as possible using a toothpick. After the grease was spread with a toothpick, excess was wiped off with a paper towel, leaving an extremely thin layer. This was the best available method to minimize the chance that grease would get on the nanoindenter tip. A sharpie was used to label the slide and create quadrants and different shapes on the slides to make it easier to find broken microsphere again in the SEM as seen in Figure 21. 


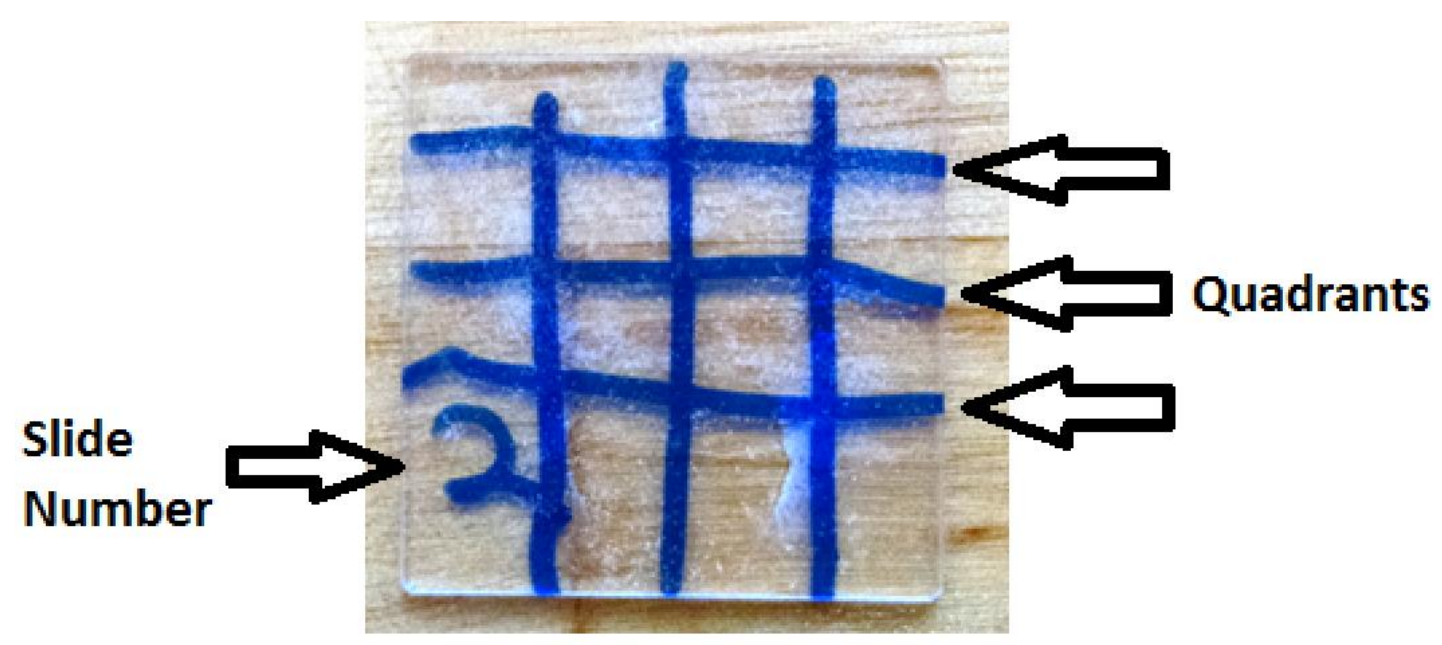

Figure 21: Fused Silica Slide that is Labeled and marked

A Q-tip was used to remove a small amount of microspheres from their container as seen in Figure 22. The microspheres were deposited onto the substrate by gently tapping the Q-tip over the substrate. Once a sample was prepared it was attached to the sample holder.

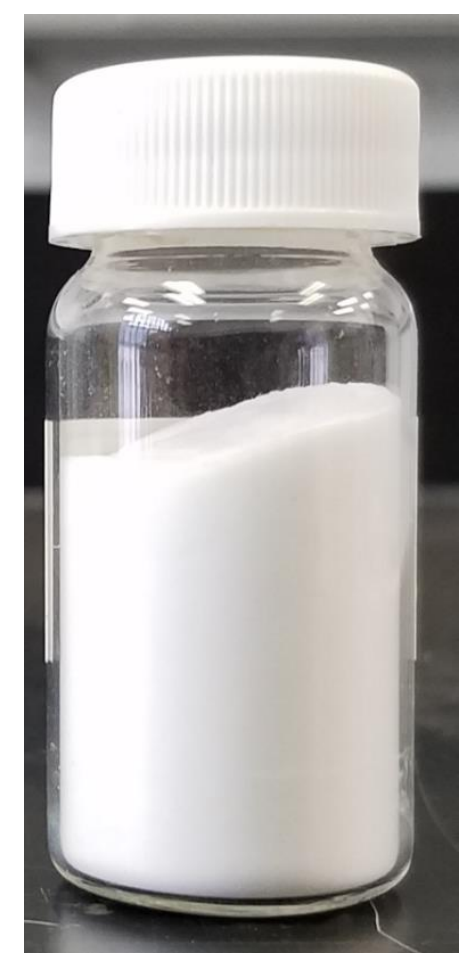

Figure 22: Container of Microspheres 
The sample holder was an aluminum stub with alumina slides glued to each side. This raised the sample off of the holder and provided a gap to slide a thin object like a knife blade underneath to remove the sample after testing was complete as seen in Figure 23 and Figure 24. Alumina was used because it is a strong ceramic that should not interfere with the results. Samples were attached to it using a small drop of Zap-A-Gap adhesive on each piece of alumina. The effect of the Zap-A-Gap on the results is minimal and already taken into account by the nanoindenter.

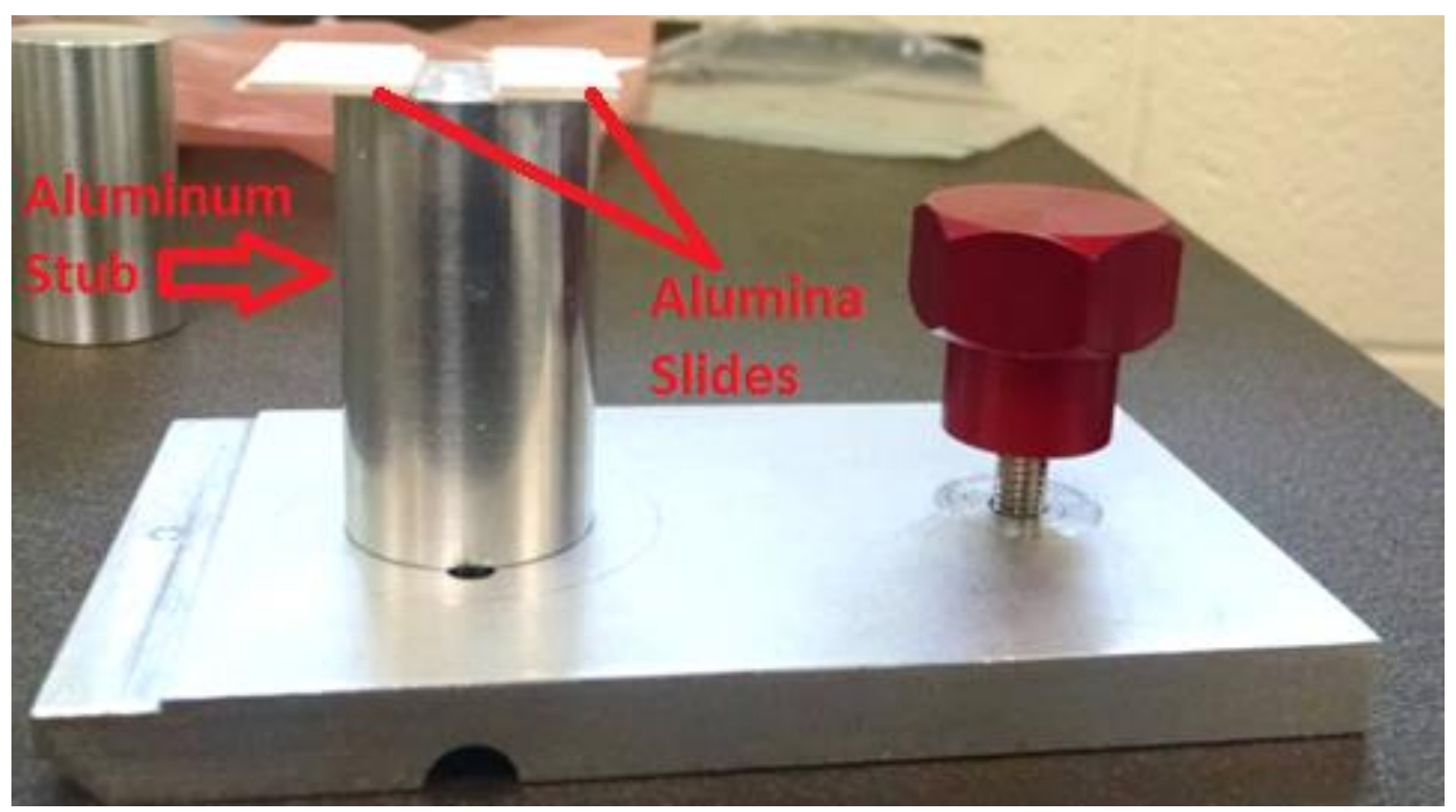

Figure 23: Sample Holder 


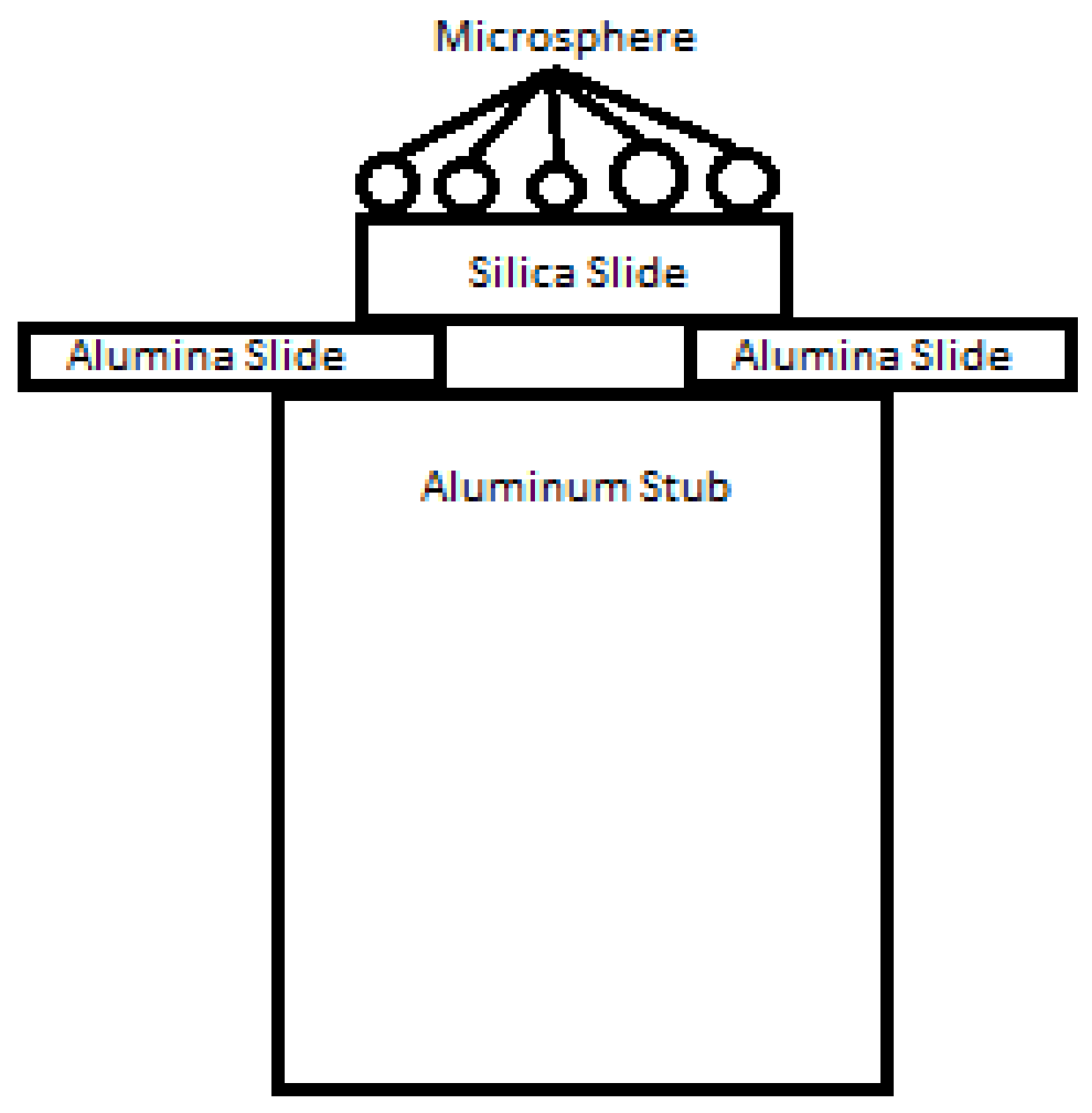

Figure 24: Nanoindenter Sample Schematic

\section{4: Nanoindentation of Microspheres}

Nanoindentation is normally used to measure various mechanical properties of a material without causing substantial damage. Usually a sharp tip is used to impact a sample and measure a material's resistance to indentation. Testing intact microspheres with a sharp tip is difficult because the sharp tip usually slides off the microsphere without indenting it as seen in Figure 25. 


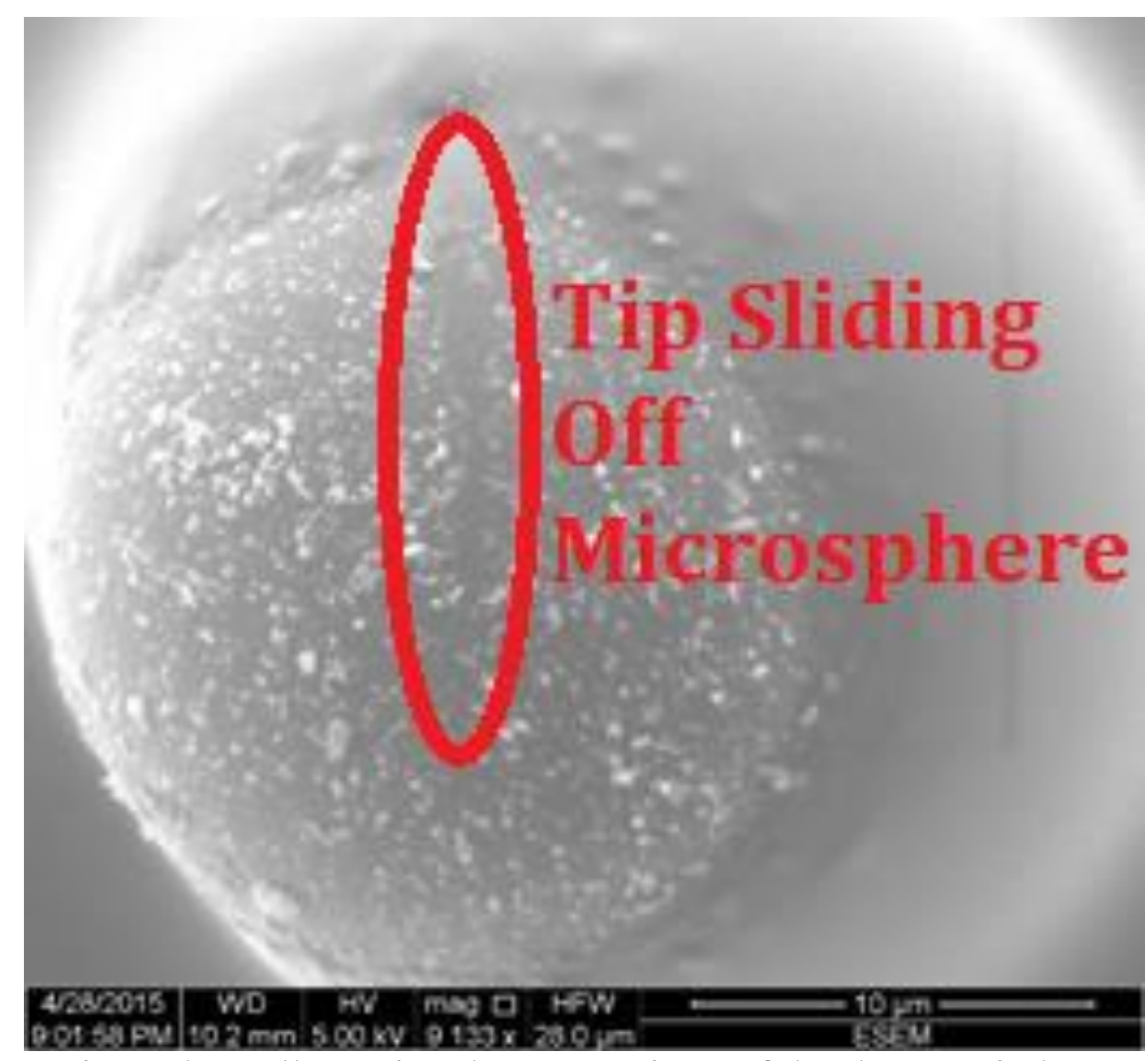

Figure 25: Microsphere Illustrating the Impression Left by the Nanoindenter Sharp Tip Before Sliding Off.

To test intact microspheres, a 100 micron flat end diamond tip from Micro Materials was used to crush the microsphere. This tip acted like a flat plate and was large enough to prevent microspheres from rolling out from under it as long as they were not on the edge of the tip. Individual microsphere was crushed between the tip end and the substrate as shown in Figure 26. For these experiments, a Micro Materials NanoTest instrument was used. The instrument could apply forces up to $500 \mathrm{mN}$ to a sample and indent to a distance of 20 microns with resolutions of $3 \mathrm{nN}$ and $0.001 \mathrm{~nm}$, respectively [52]. This was far more than any force required in the nanoindentation papers discussed earlier and the high level of resolution provided better data [32]. 


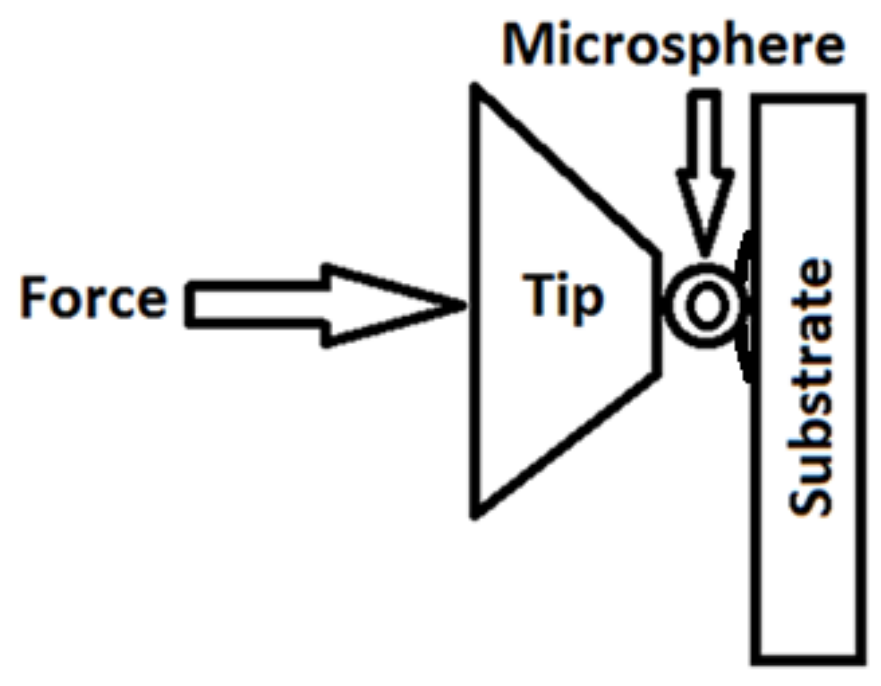

Figure 26: Schematic of a Flat Tip Nanoindenter Crush Test

The Micro Materials unit is regulated at a temperature of $24.80^{\circ} \mathrm{C}$ and is set on a concrete slab that is separate from the foundation of the building. This reduced the effect that external vibrations had on data collection. The indentation location is determined using the microscope attached to the unit. The microscope has 4x, 10x, 20x, and 40x objectives. The 4x and 10x objectives were used to find microspheres suitable for crush tests. The 20x objective was used to aim the indenter tip.

The stage control system is used to move the sample stage around under the objective and into focus. The stage can move up to 250 microns/s and allows the user to move the stage along a single axis or combination of axes in system defined positive and negative directions. Backlash must be removed from the system if the user changes the direction on the axis for the stage. The screen shows if contact with the tip has been made, what direction the stage is moving, the position of the tip in each axis, and the displacement of the tip for each axis. When samples are in focus for the 20x objective and aligned in the crosshairs, the indenter's software calculates roughly how far away the sample surface is from the tip and where to move the 
sample to indent the correct point. Samples need to be focused on this objective so the instrument can find the surface of the material for indentation. After sample preparation was completed, optical micrographs were taken of the positions of the microspheres to correlate the experiment results to specific microspheres. After testing, the microspheres were examined again with the microscope to confirm crushing. The microspheres were then examined one last time using an SEM to analyze the wall thickness.

Experiments were set up using the nanoindenter's computer. The computer allowed the user to view the digital output to the nanoindenter's microscope, change what objective the microscope uses, access sample stage control, calibrate various nanoindenter settings, perform maintenance checks, complete experiment setup, and view the results. To ensure the accuracy and functionality of the nanoindenter, multiple calibrations needed to be completed. When switching out tips, the microscope calibrations for cross hair accuracy and sample stage position were performed. These calibrations make it possible for the nanoindenter to accurately align a point on the sample with the nanoindenter tip and determine the distance a sample will be from the tip when moved from the microscope to the tip. Figure 27 shows the different ways provided by the nanoindenter to conduct an indentation experiment.

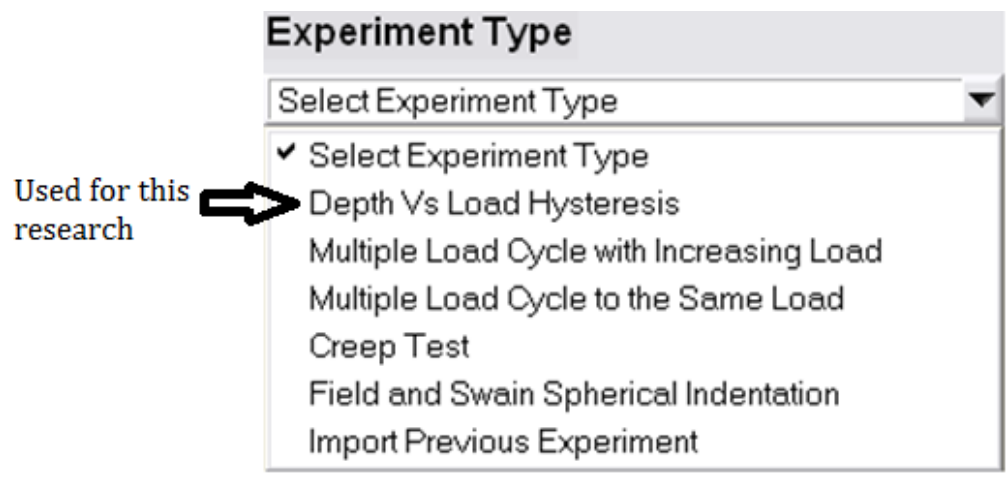

Figure 27: Indentation Experiment Type 
Depth vs. load hysteresis was the method used to conduct indentation experiments. Experiments were terminated when they hit either the limit to the nanoindenter depth or $300 \mathrm{mN}$. The load maximum was chosen as a way to minimize the time it would take the system to reach maximum load if the tip made contact with the substrate and could not reach maximum depth. It was also set high enough that the indenter should never hit maximum load before breaking a microsphere. Initially the load rate was set to $10 \mathrm{mN} / \mathrm{s}$. This worked for everything but the PWHGMs. The load required to break the PWHGMs was small enough that they would break before the indenter could collect many data points, making results hard to evaluate. To counter this problem, the load was lowered to $1 \mathrm{mN} / \mathrm{s}$, giving the nanoindenter time to collect enough data points to make the data readable. Because of the difficulty of finding an individual microsphere and aiming at it properly, only one indent was completed with each schedule.

\section{4: Interpretation of Nano-Crush Test Results}

Data collected from nanoindentation was used to determine the failure load using the method based on previous nanoindentation work by Carlisle et al [32]. The stress acting on the microsphere was calculated using Equation (21) from the analysis of a hollow sphere between two flat plates by Rumbarger et al [53]. Results from previous nanoindentation work on microspheres showed a tip displacement vs applied load graph with three regions. Each region was marked by a large change in the slope of the line formed from the data as seen in Figure 28. When examining the first two regions, data usually takes the form of a normal stress vs. strain curve. That meant that the load at the slope change is the fracture load of a microsphere. The final region of the graph shows when the tip of the nanoindenter either impacts the substrate surface or hits its max depth and then reduces load. In reality, there are a number of variations that can occur. 


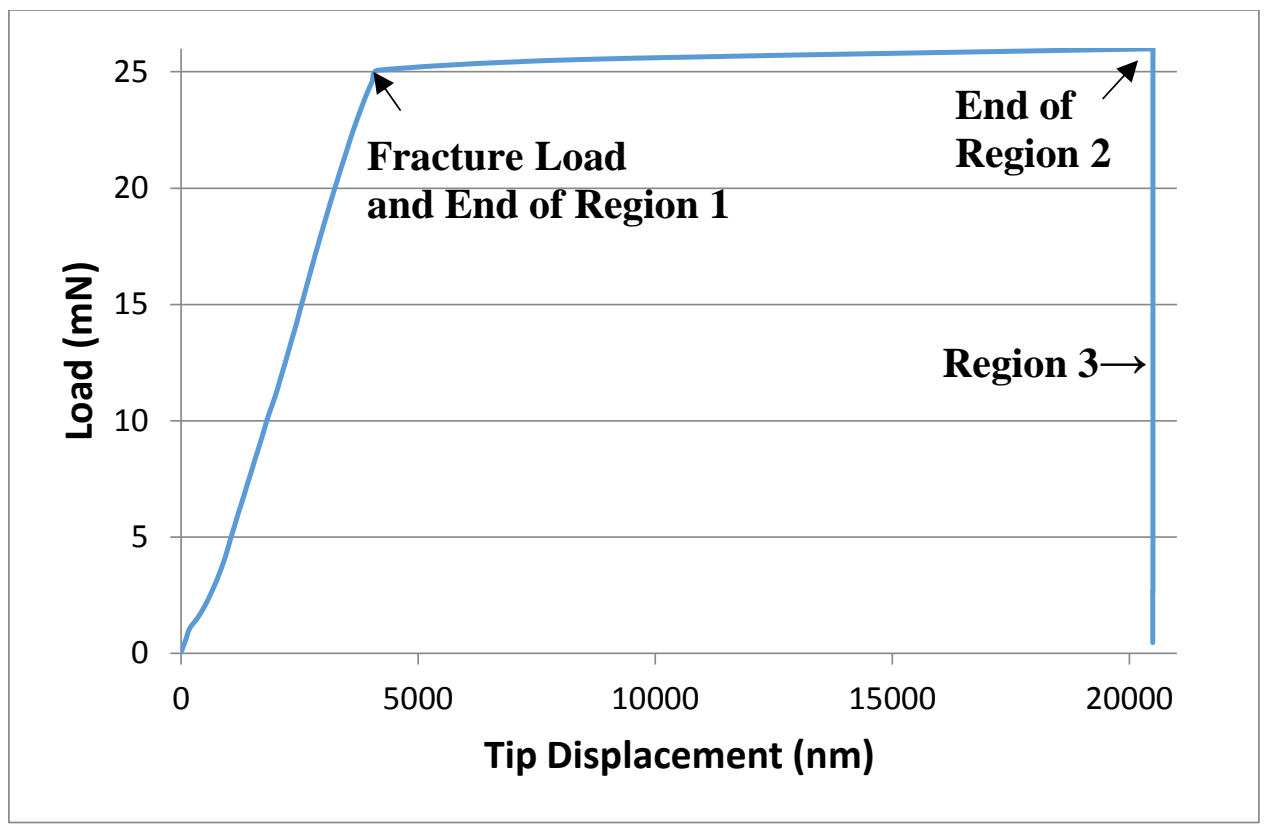

Figure 28: Typical Nanoindenter Test Results (3M S38HS Sample)

The stress causing failure in the microspheres due to the compressive forces acting on the microspheres is referred to as the crush strength. Under uniaxial loading, multiple types of stress will act upon the sphere at once. Due to the complexity of the stress distribution on the sphere, there is no consensus on how to solve the crush strength of a thin wall hollow sphere under uniaxial compression. Although there are a few models that attempt to provide a solution, the accuracy of the models is questionable or the test methodology is not applicable to this research [54], [55].

In the present investigation, the analysis of the crush strength was simplified using multiple assumptions. First, the Weibull equation was simplified by assuming stress acting on the microspheres was uniaxial uniform compression meaning $\mathrm{k}$ in Equation (19) would be 1. Second, microspheres within a ten micron range were assumed to have approximately the same volume. This means that data points within the designated range do not need to be converted 
from one volume to another if their diameters are a few microns apart. Third, microspheres of the same volume had roughly the same number of flaws and larger microspheres had a larger number of flaws. Forth, the crush strength of a microsphere was defined as the stress at failure of the mid-wall of the microsphere at the equator using Equation (21). This is the same equation that Rumbarger et al [53] used to calculate compressive stress for comparison to their finite element results (FEA) which modeled the compressive and bending stresses acting throughout the microsphere. The results from the equation compared favorably with the FEA. In Equation (21) $\sigma$ is the crush strength, $F$ is the force applied at failure, $r$ is the outer radius, and $t$ is the wall thickness.

$$
\sigma=\frac{F}{\pi\left(r^{2}-(r-t)^{2}\right)}
$$

Even if this is not the best equation for calculating the crush strength of a microsphere, it does include multiple variables that are important in calculating the stress. Unlike some of the methods discussed earlier, Equation (21) uses both the radius of the microsphere and the wall thickness when calculating the stress on the microsphere. Even though these assumptions may not provide the most accurate analysis, they do provide a semi-quantitative comparison on how the diameter, wall thickness, and different stages of production affect the strength of the microspheres. With these assumptions the Weibull analysis was simplified to the basic equations for uniaxial uniform compression. 


\section{Ch. 6: Results and Discussion}

To confirm that a microsphere was broken, before and after images were taken using the microscope attached to the nanoindenter system. If the test was successful, then there was a clear change in appearance from the before the test to after the test as seen in Figure 29. Most successful tests resulted in the microsphere being completely crushed or clearly broken into multiple parts without any of the surrounding microsphere being affected. The output of each test was plotted in excel. As discussed earlier, the nano crush test results have three sections. The first two sections usually look like a typical stress vs. strain curve with a few exceptions. The third section is the nanoindenter removing the force on the tip as it hit the max tip displacement. Figure 30 and Figure 31 show the different types of nano crush test results. In the basic stress vs. strain curve shown in Figure 30, the microsphere was placed on a thin region of grease where it did not need to move through the grease to make contact with the substrate. This type of result was the most common ones observed. Figure 31 presents the most common variation where the nanoindenter tip must force the microsphere through grease before contact is made with the substrate. The first change in slope indicates where deformation on the microsphere begins. Additional types of nano crush test results are included in Appendix D.
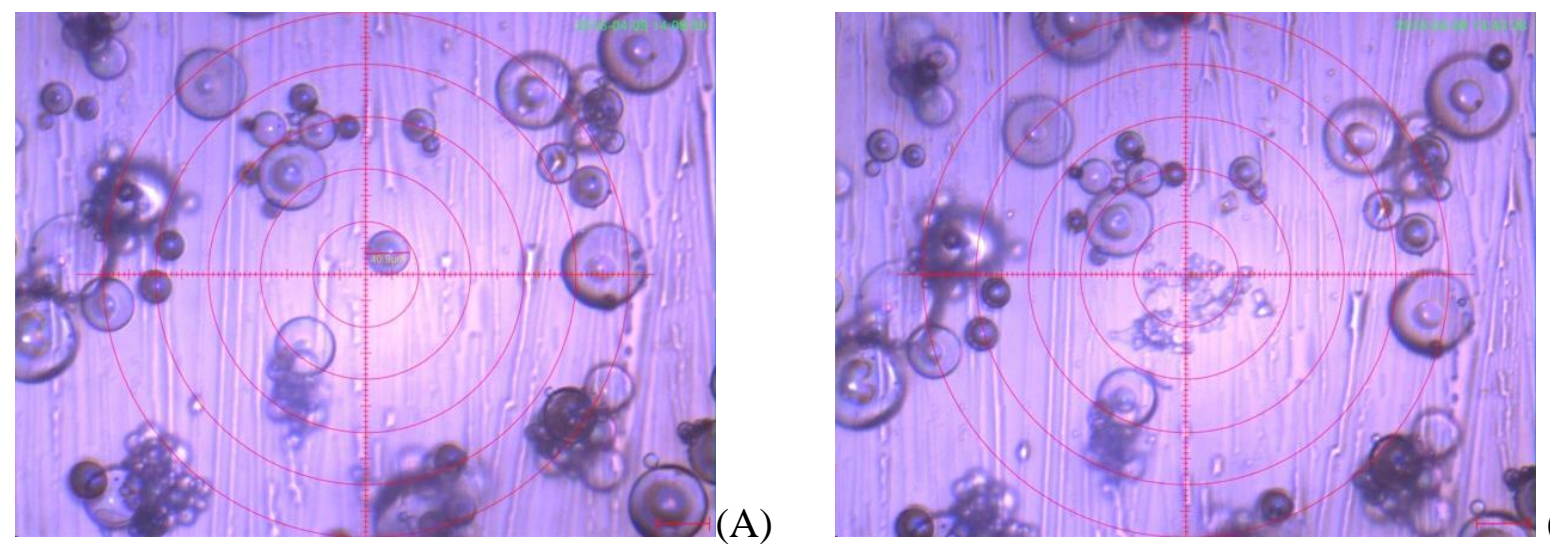

(B)

Figure 29: Microsphere before Test (A); Broken Microsphere after Test (B) 


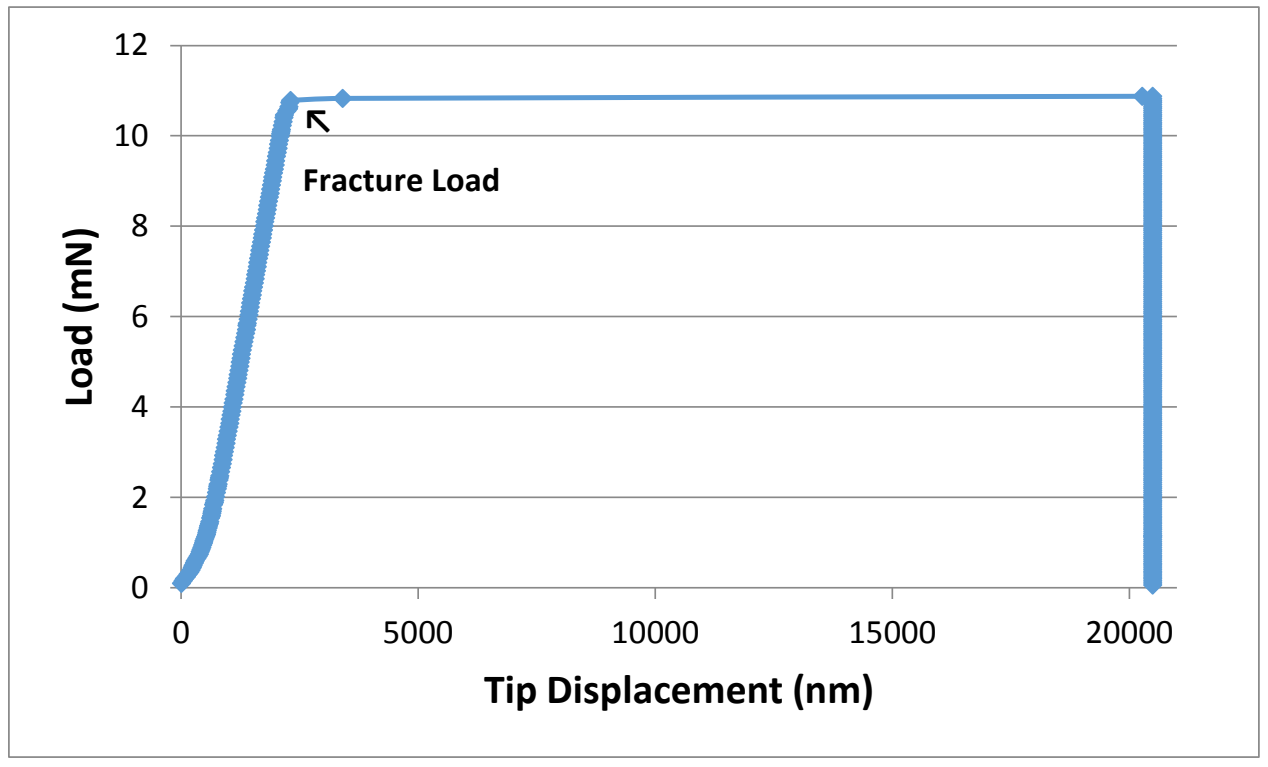

Figure 30: Normal Nano Crush Test Result

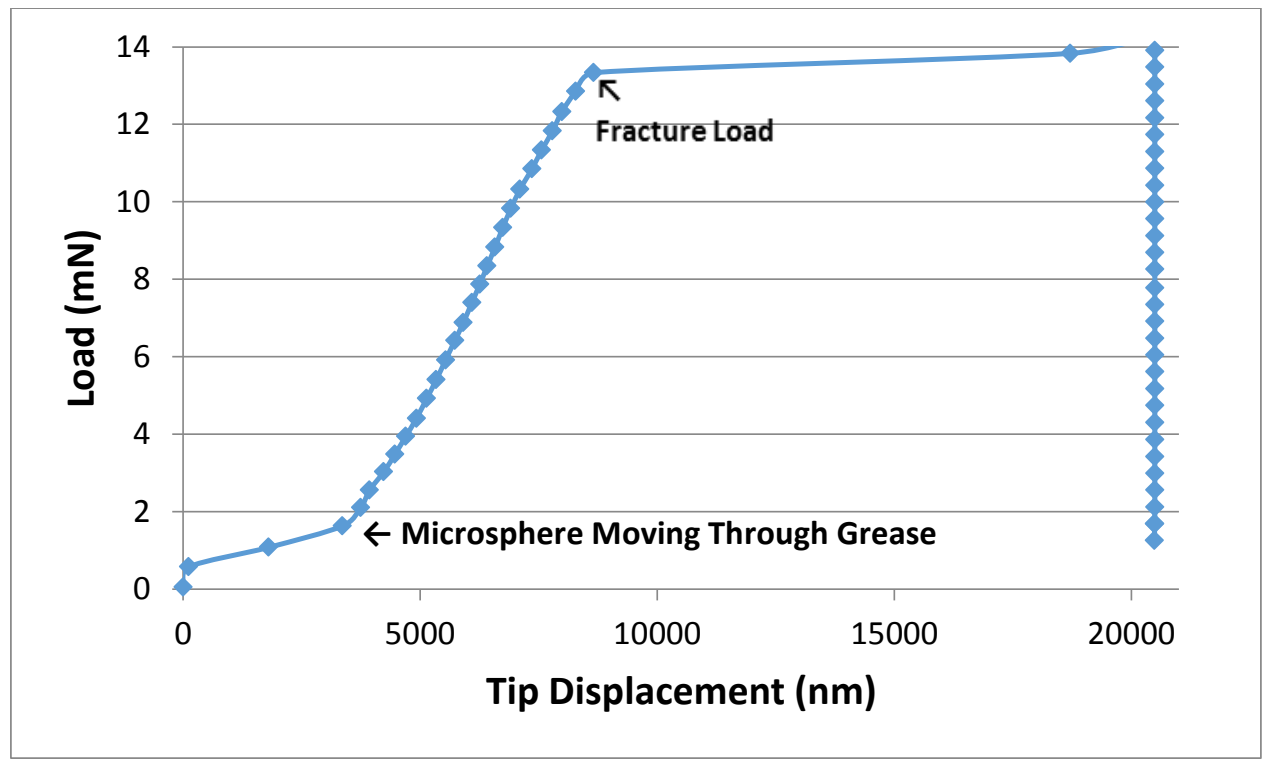

Figure 31: Microsphere Initially Moving Through Grease 
Micrographs of broken microspheres were taken using an environmental SEM (E-SEM) and field emission SEM (FE-SEM) at the Nanoscale Characterization and Fabrication Laboratory (NCFL). The microspheres were coated with gold nano particles to reduce the charging of the microspheres inside the SEM. With the micrographs, it was possible to measure the diameter and wall thickness of 18 ARC HGMs and PWHGMs. For each microsphere there were usually two images recorded as seen in Figure 32. One was of the entire microsphere so the diameter could be measured and one of the wall thickness. Multiple measurements were made on the wall due to the fact that the wall thickness is not constant throughout a microsphere. The average wall thickness is displayed in Table 3 alongside the corresponding microsphere diameter. Also included in Table 3 are the average wall thicknesses of all measured microspheres and microspheres in the $30-40$ micron range. Since there was not enough data to calculate the wall thickness for each diameter range, the total average wall thickness was used in all stress calculations. The different layers of the PWHGM walls are not separated when measuring the wall thickness. Table 4 and Table 5 show the wall thickness measurements for the 3M S38HS and 3M S60HS microspheres, respectively. At the bottom of each table, the average wall thickness calculated from using all the measured microsphere walls is shown. 

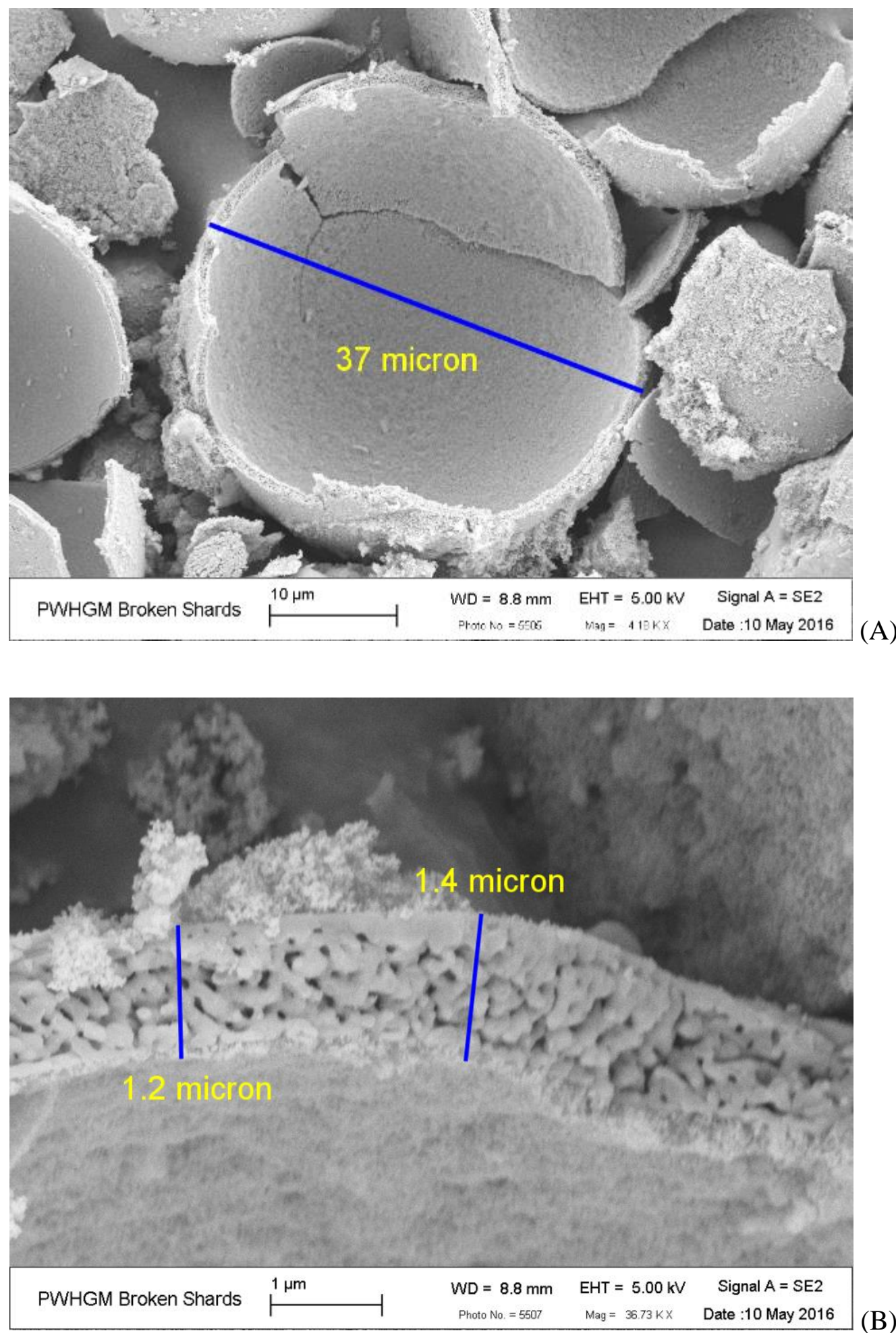

Figure 32: Measured PWHGM Half-Shell (A); Measured PWHGM Wall (B) 
Table 3: Microsphere Diameter and Corresponding Wall Thickness

\begin{tabular}{|c|c|}
\hline $\begin{array}{l}\text { Microsphere Diameter } \\
\text { (microns) }\end{array}$ & Wall Thickness (microns) \\
\hline 23 & 0.62 \\
\hline 28 & 0.63 \\
\hline 28 & 0.88 \\
\hline 29 & 0.57 \\
\hline 30 & 0.48 \\
\hline 31 & 0.55 \\
\hline 32 & 0.68 \\
\hline 34 & 0.92 \\
\hline 34 & 0.77 \\
\hline 37 & 1.30 \\
\hline 38 & 1.03 \\
\hline 39 & 0.88 \\
\hline 40 & 0.90 \\
\hline 42 & 0.72 \\
\hline 61 & 0.65 \\
\hline 79 & 1.15 \\
\hline 88 & 0.96 \\
\hline 110 & 1.44 \\
\hline Average total Wall Thickness & 0.80 \\
\hline $\begin{array}{l}\text { 30-40 micron Diameter } \\
\text { Microspheres }\end{array}$ & 0.80 \\
\hline
\end{tabular}


Table 4: 3M S38HS Microsphere Diameter and Corresponding Wall Thickness

\begin{tabular}{|c|c|}
\hline $\begin{array}{c}\text { Microsphere Diameter } \\
\text { (Microns) }\end{array}$ & Wall Thickness (microns) \\
\hline 34 & 0.69 \\
\hline 36 & 0.65 \\
\hline 37 & 0.63 \\
\hline 37 & 0.83 \\
\hline 44 & 0.65 \\
\hline 3M S38HS Average Wall \\
Thickness & 0.70 \\
\hline
\end{tabular}

Table 5: 3M S50HS Microsphere Diameter and Corresponding Wall Thickness

\begin{tabular}{|c|c|}
\hline $\begin{array}{c}\text { Microsphere Diameter } \\
\text { (microns) }\end{array}$ & Wall Thickness (microns) \\
\hline 23 & 0.76 \\
\hline 24 & 0.86 \\
\hline 26 & 0.73 \\
\hline 27 & 0.90 \\
\hline 36 & 1.06 \\
\hline $\begin{array}{c}\text { 3M S60HS Average Wall } \\
\text { Thickness }\end{array}$ & 0.90 \\
\hline
\end{tabular}


Fracture stress was computed from Equation (21) using measured diameters and wall thicknesses. Stress for each test was rank ordered from lowest to highest and the experimental probability of failure was calculated using Equation (17) where $n$ was the rank order of a specific stress as seen in Table 6. Data was separated into different sets based on microsphere diameter and type of microsphere. Data such as those presented in Table 6 were used to generate all Weibull plots. Linear regression was used to extrapolate the Weibull equation for each data set.

Table 6: Calculation of Probability of Failure. This table shows how the probability of failure was calculated by rank ordering the Samples from lowest to highest fracture stress. The value of $N$ for this set of data was 10. The set shown are the 60-70 micron ARC HGMs.

\begin{tabular}{|c|c|c|}
\hline Rank Order $(n)$ & $\begin{array}{c}\text { Experimental Probability of } \\
\text { Failure, } P_{f} \\
P_{f}=\frac{n}{N+1}\end{array}$ & \\
\hline 1 & 0.09 & \\
\hline 2 & 0.18 & 1,700 \\
\hline 3 & 0.27 & 2,450 \\
\hline 4 & 0.36 & 2,475 \\
\hline 5 & 0.45 & 2,825 \\
\hline 6 & 0.54 & 2,950 \\
\hline 7 & 0.63 & 4,360 \\
\hline 8 & 0.72 & 4,615 \\
\hline 9 & 0.81 & 5,085 \\
\hline 10 & 0.91 & 9,085 \\
\hline
\end{tabular}


Figure 33 through Figure 35 show the effects of the microsphere diameter on the strength of the microspheres for each stage of PWHGM production. Figure 36 and Figure 37 show the results of three parameter analyses on PWHGMs where the threshold parameter, $\sigma_{\mu}$, was set to 270 psi for the $30-40$ micron diameter range and 300 psi for the $40-50$ micron diameter range. Figure 38 shows the Weibull analysis results when Equation (7) was used to calculate stress. Figure 39 through Figure 41 show how the strength of the microspheres changed during each stage of production for a specific diameter range. Finally, Figure 42 presents the Weibull results for the $3 \mathrm{M}$ microspheres with along with the crush strength values provided by $3 \mathrm{M}$. The alternative axes on each graph show the stress (ksi) and probability of failure (\%).

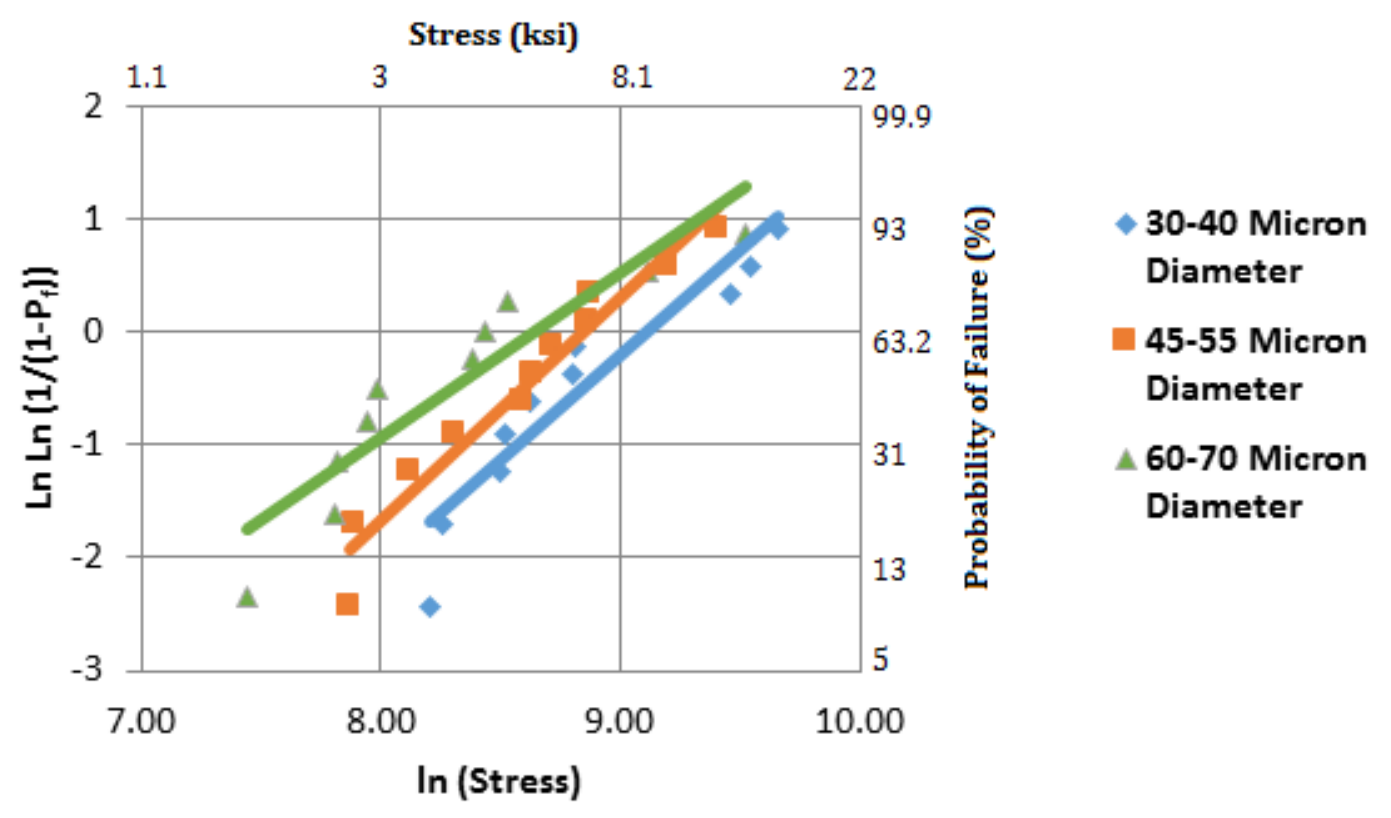

Figure 33: ARC HGM Weibull Analysis 


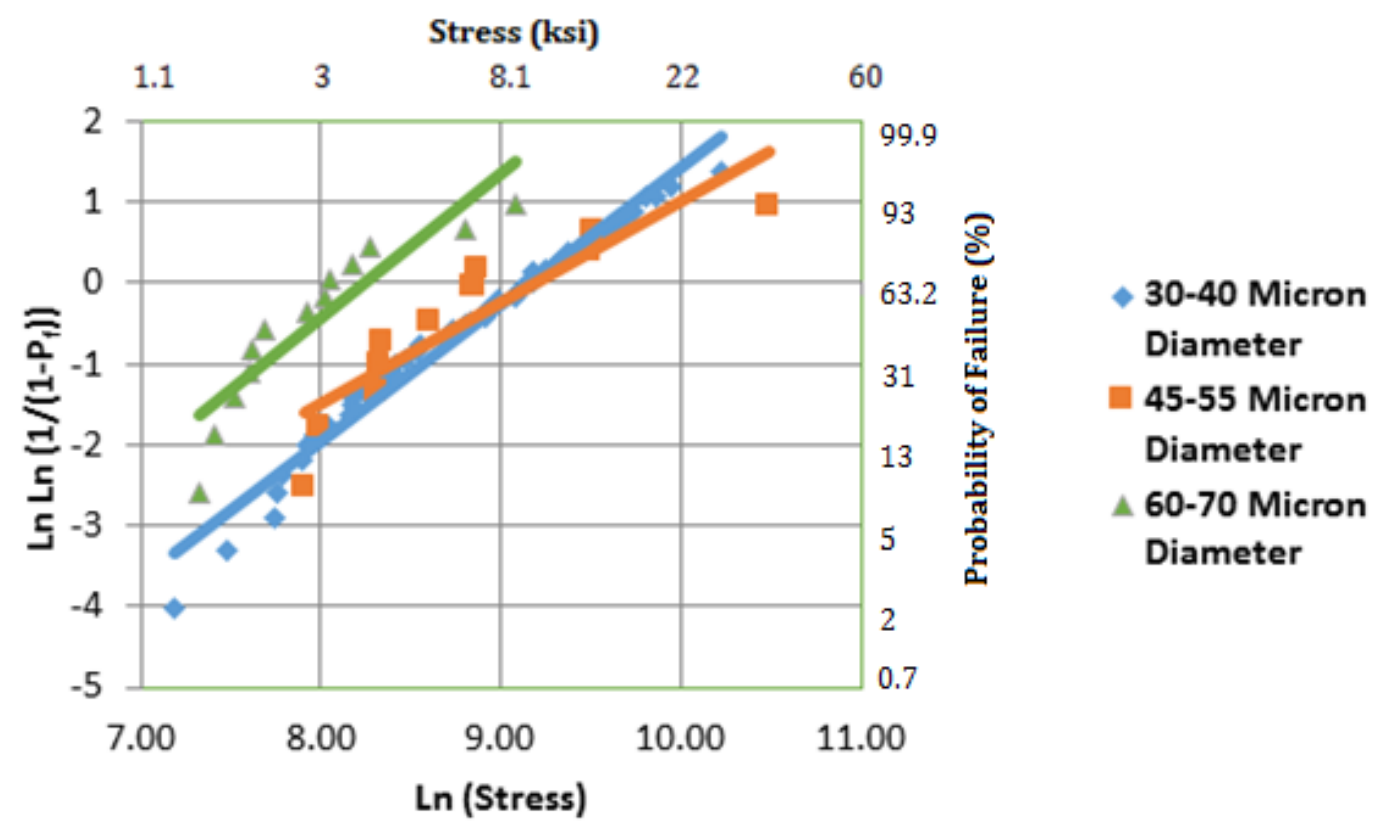

Figure 34: Heat Treated ARC HGM Weibull Analysis

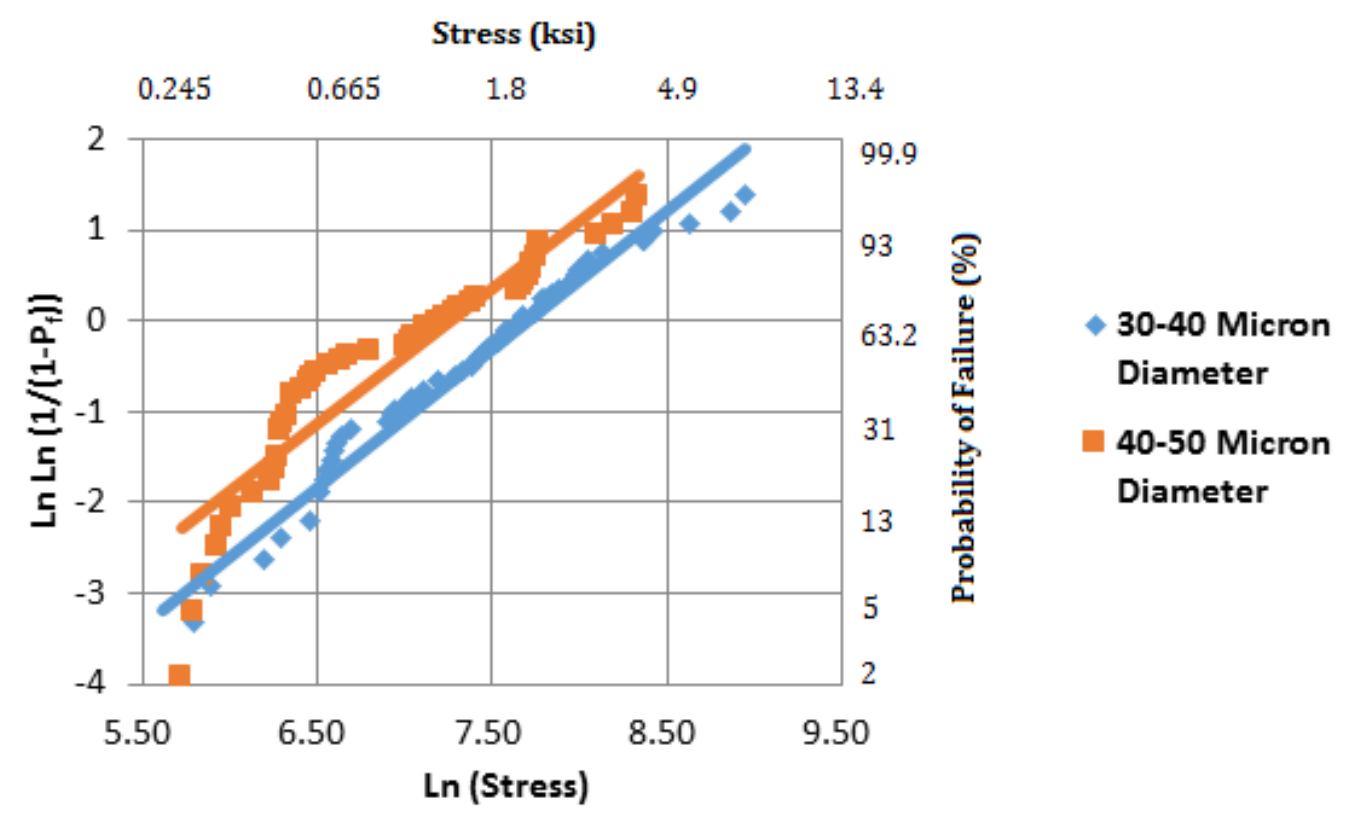

Figure 35: ARC PWHGM Weibull Analysis 


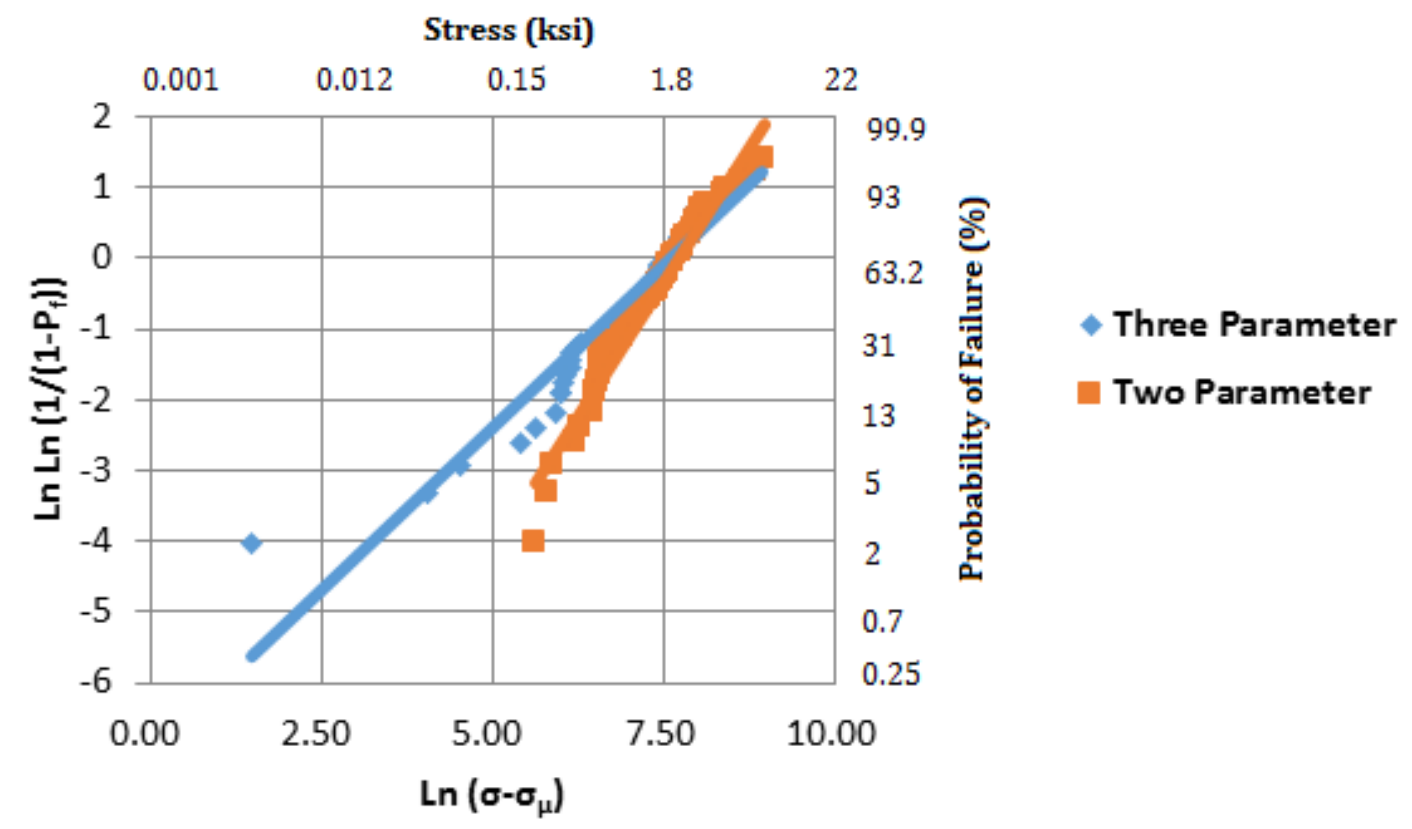

Figure 36: ARC PWHGM Three Parameter Weibull Analysis Using $\sigma_{\mu}=270$ psi vs. Two Parameter Results where $\sigma_{\mu}=0$

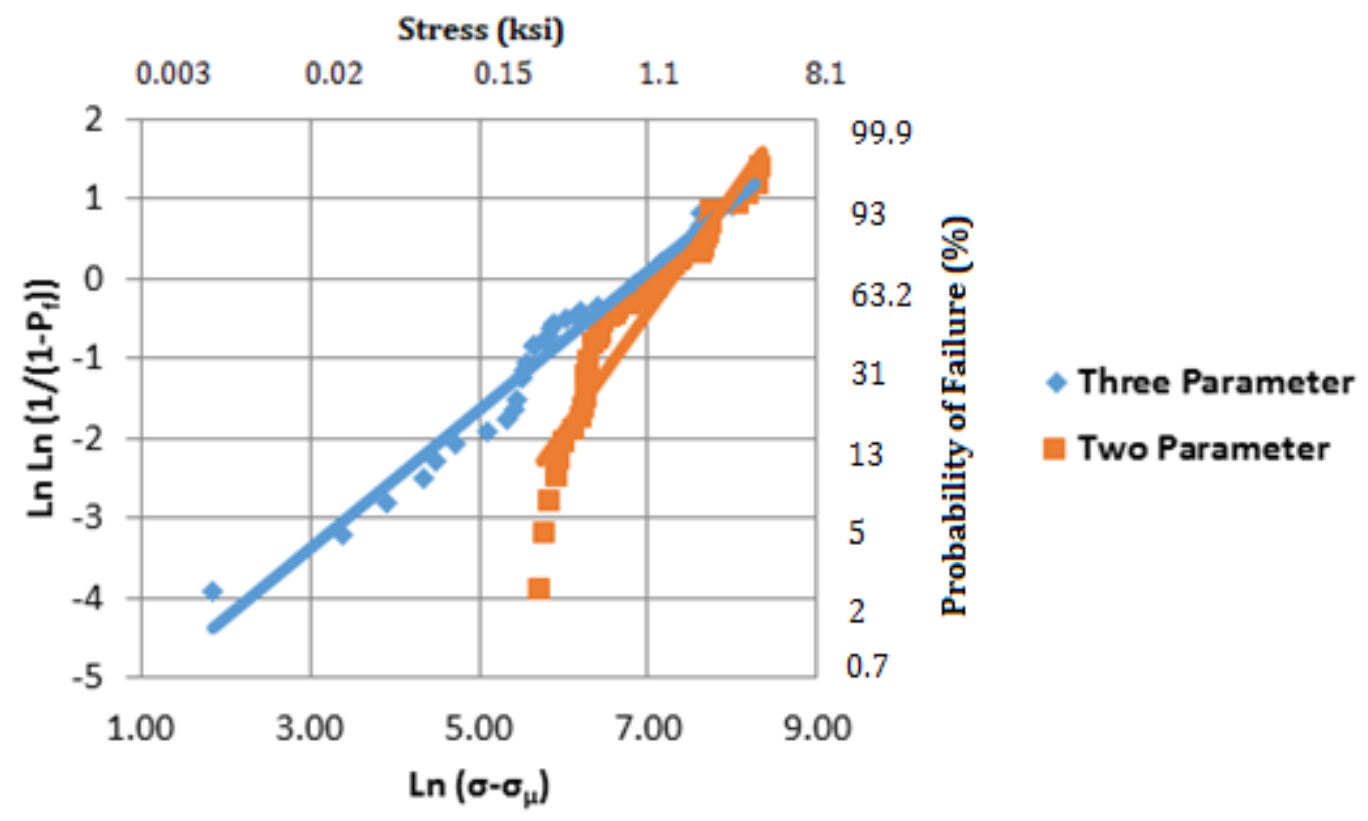

Figure 37: ARC PWHGM Three Parameter Weibull Analysis Using $\sigma_{\mu}=300$ psi vs. Two Parameter Results where $\sigma_{\mu}=0$ 


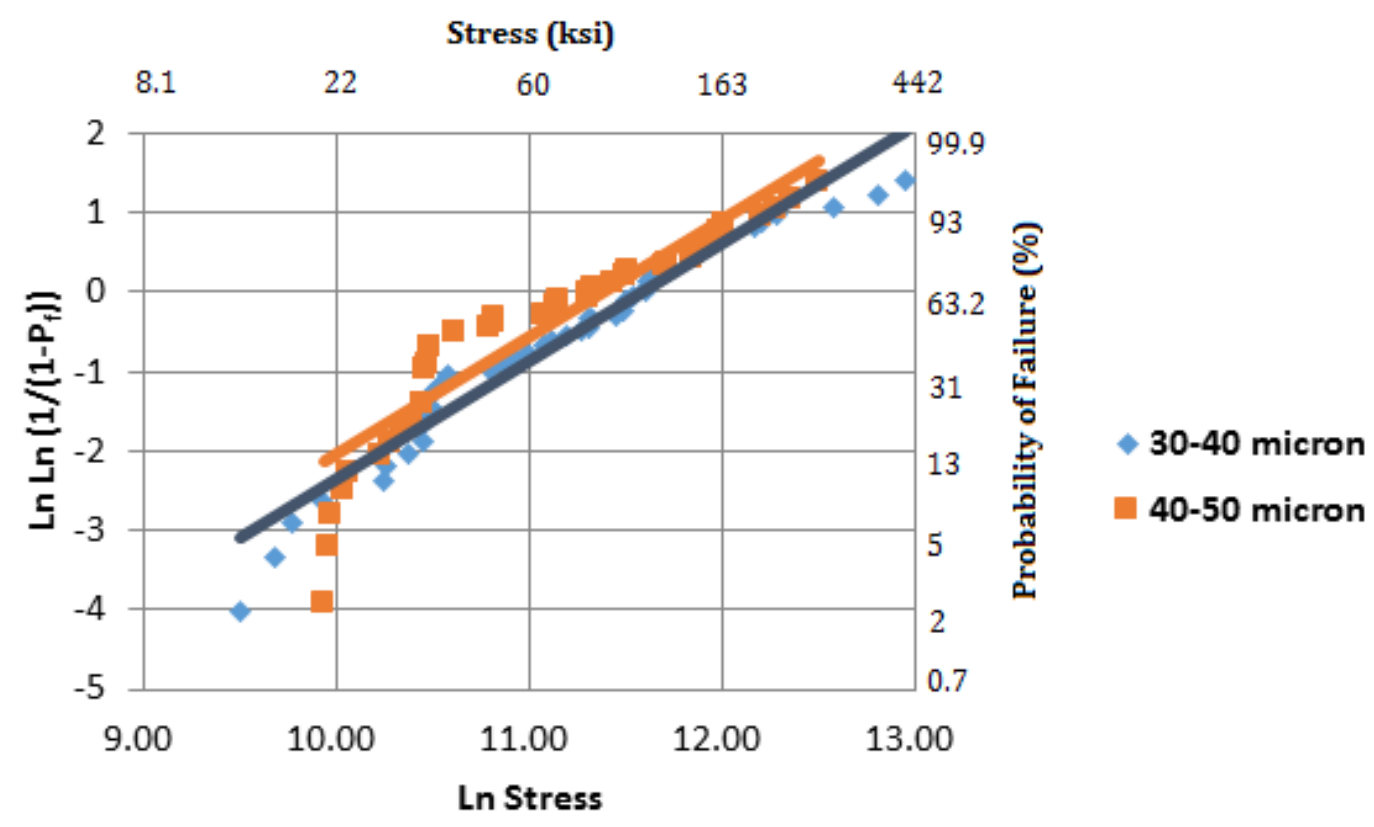

Figure 38: PWHGMs Calculated Using Equation (7)

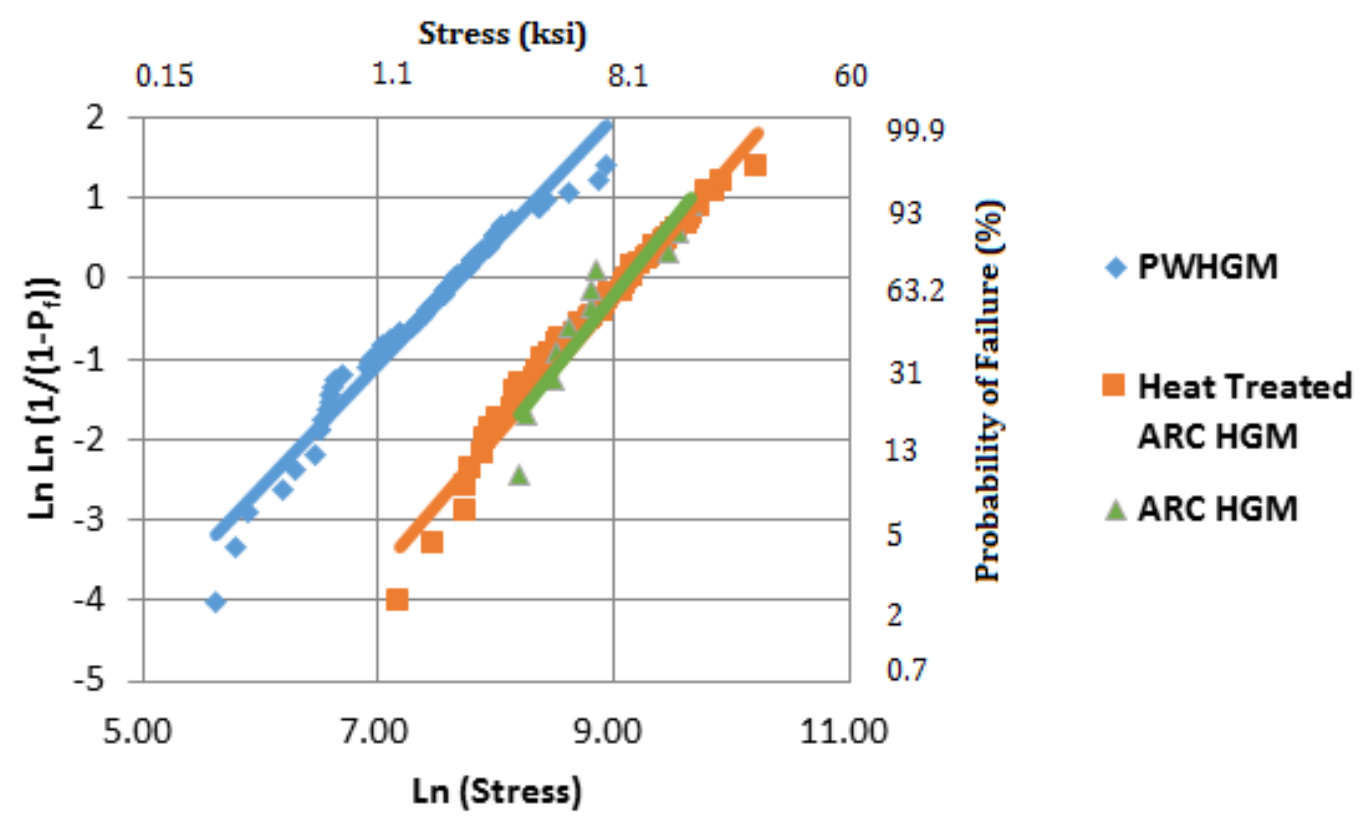

Figure 39: Effects of the Different Stages of PWHGM Production on Strength for 30-40 Micron Microspheres 


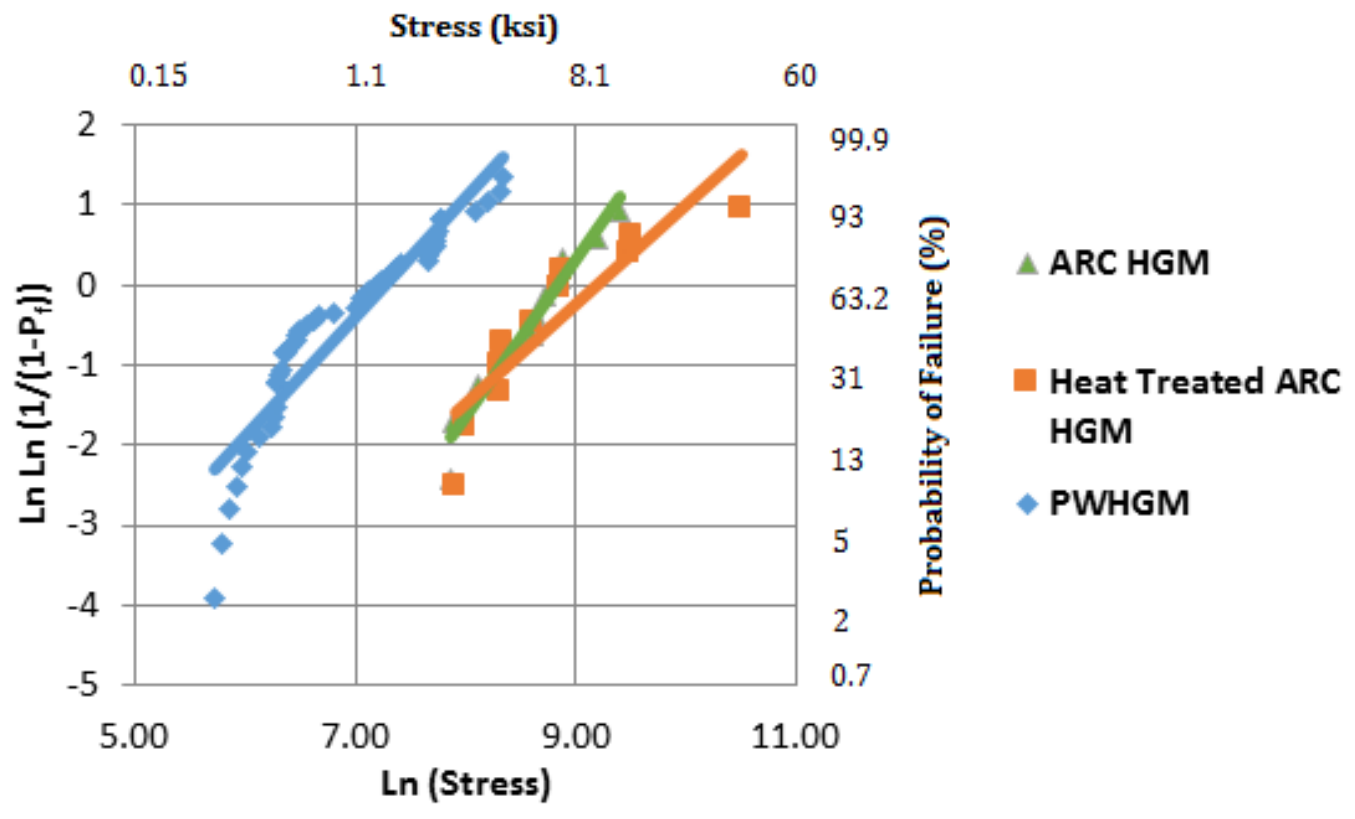

Figure 40: Effects of the Different Stages of PWHGM Production on Strength for 45-55 Micron Microspheres

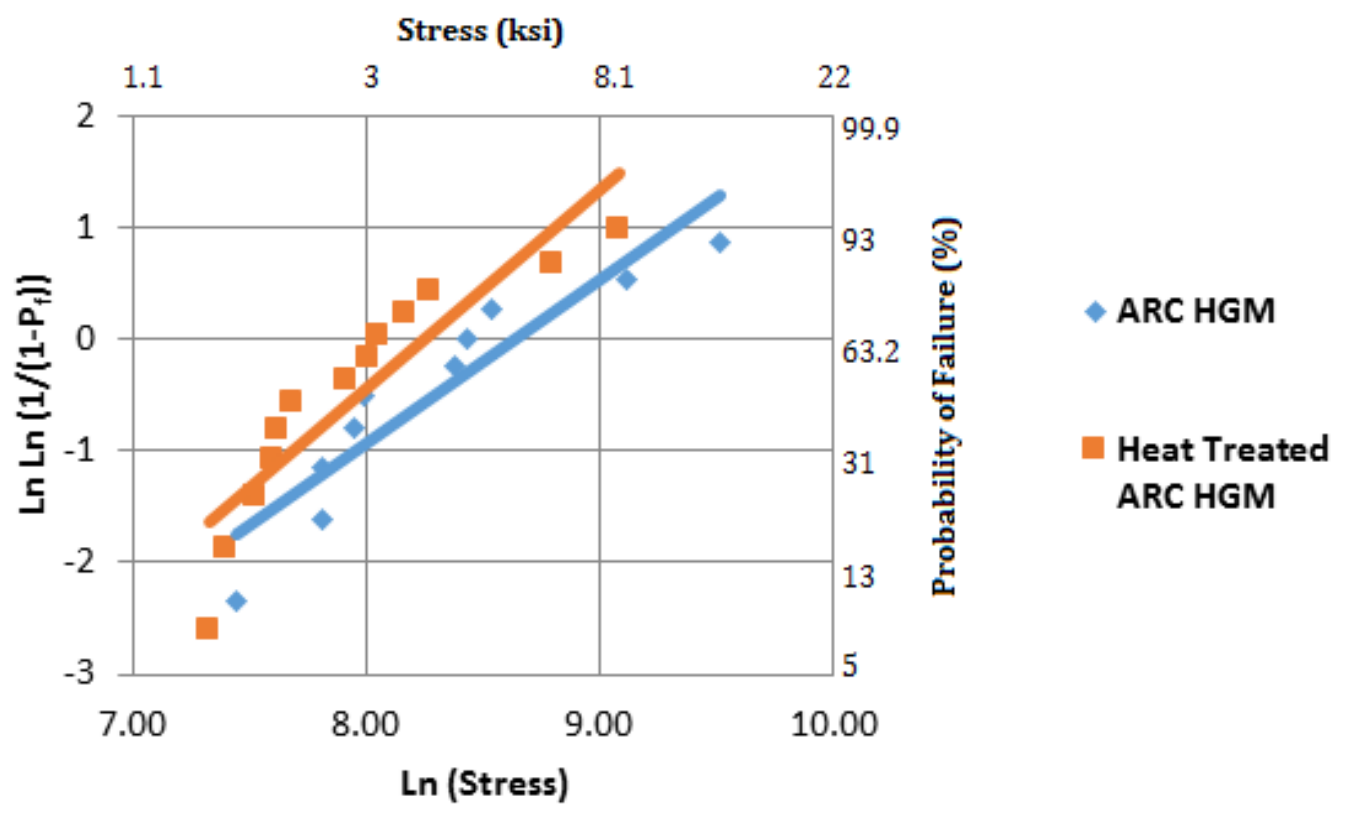

Figure 41: Effects of the Different Stages of PWHGM Production on Strength for 60-70 Micron Microspheres 


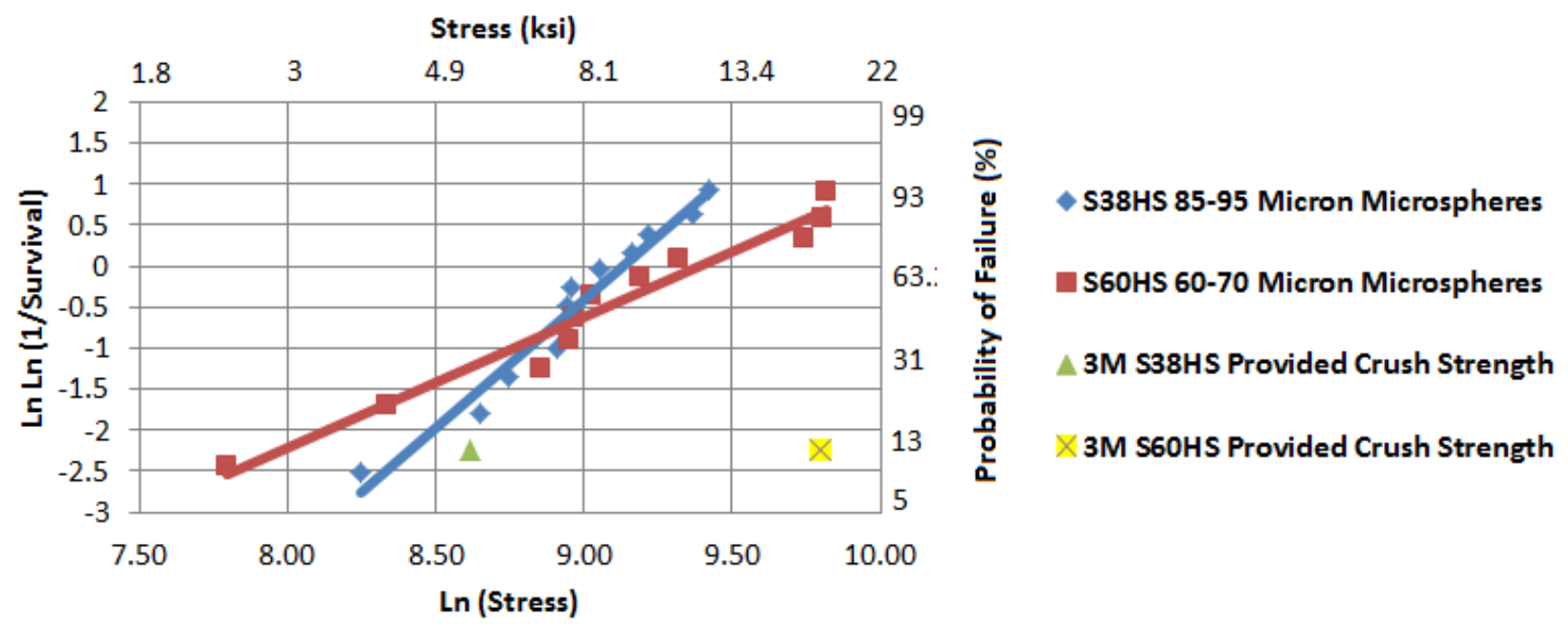

Figure 42: 3M S38HS and S60HS Microspheres

Table 7 presents the results of the Weibull analysis for all data sets. The table is divided into multiple sections based on the type microsphere. For each data set, the Weibull equation, Weibull modulus, characteristic strength, and $\mathrm{r}^{2}$ value are provided. From the plots and table, one can predict the likelihood that a microsphere will fail at a specific stress, determine the amount of scattering in a particular data set, look at the strength required to cause most microspheres to fail, and see the accuracy of the linear regression on a particular data set. 
Table 7: Weibull Analyses for Each Data Set

\begin{tabular}{|c|c|c|c|c|c|}
\hline $\begin{array}{c}\text { Type of } \\
\text { Microsphere }\end{array}$ & $\begin{array}{l}\text { Microsphere } \\
\text { Diameter }\end{array}$ & $\begin{array}{l}\text { Weibull } \\
\text { Equation }\end{array}$ & $\begin{array}{c}\text { Weibull } \\
\text { Modulus (m) }\end{array}$ & $\begin{array}{l}\text { Characteristic } \\
\text { Strength }\left(\sigma_{0}\right)\end{array}$ & $r^{2}$ \\
\hline \multirow{4}{*}{ ARC HGMs } & $\begin{array}{c}\text { 30-40 microns } \\
\text { (35 micron) }\end{array}$ & $\begin{array}{c}y=1.86 x \\
-16.95\end{array}$ & 1.86 & 9072 psi & 0.86 \\
\hline & $\begin{array}{c}45-55 \text { microns } \\
\text { (50 micron) }\end{array}$ & $\begin{array}{c}y=1.98 x \\
-17.50\end{array}$ & 1.98 & $6894 \mathrm{psi}$ & 0.95 \\
\hline & $\begin{array}{c}\text { 60-70 microns } \\
\text { (65 micron) }\end{array}$ & $\begin{array}{c}y=1.46 x \\
-12.60\end{array}$ & 1.46 & $5598 \mathrm{psi}$ & 0.86 \\
\hline & $\begin{array}{c}\text { Combined ARC } \\
\text { HGMs }\end{array}$ & $\begin{array}{c}y=2.02 x \\
-18.78\end{array}$ & 2.02 & $10906 \mathrm{psi}$ & 0.82 \\
\hline \multirow{4}{*}{$\begin{array}{c}\text { ARC Heat } \\
\text { Treated } \\
\text { HGMs }\end{array}$} & $\begin{array}{c}\text { 30-40 microns } \\
\text { (35 micron) }\end{array}$ & $\begin{array}{c}y=1.69 x \\
-15.50\end{array}$ & 1.69 & $9620 \mathrm{psi}$ & 0.97 \\
\hline & $\begin{array}{l}45-55 \text { microns } \\
\text { (50 micron) }\end{array}$ & $\begin{array}{c}y=1.25 x \\
-11.51\end{array}$ & 1.23 & $11588 \mathrm{psi}$ & 0.81 \\
\hline & $\begin{array}{c}\text { 60-70 microns } \\
\text { (65 micron) }\end{array}$ & $\begin{array}{c}y=1.79 x \\
-14.76\end{array}$ & 1.79 & $3812 \mathrm{psi}$ & 0.83 \\
\hline & $\begin{array}{c}\text { Combined HT } \\
\text { HGMs }\end{array}$ & $\begin{array}{c}y=1.68 x \\
-15.56\end{array}$ & 1.68 & $10529 \mathrm{psi}$ & 0.86 \\
\hline \multirow{3}{*}{ PWHGMs } & $\begin{array}{c}\text { 30-40 microns } \\
\text { (35 micron) }\end{array}$ & $\begin{array}{c}y=1.53 x \\
-11.78\end{array}$ & 1.53 & 2207 psi & 0.97 \\
\hline & $\begin{array}{l}40-50 \text { microns } \\
\text { (45 micron) }\end{array}$ & $\begin{array}{c}y=1.49 x \\
-10.84\end{array}$ & 1.49 & 1444 psi & 0.87 \\
\hline & Combined PWHGMs & $\begin{array}{c}y=1.53 x \\
-11.74\end{array}$ & 1.53 & $2150 \mathrm{psi}$ & 0.90 \\
\hline \multirow{2}{*}{$\begin{array}{l}\text { PWHGM } \\
\text { Three } \\
\text { Parameter }\end{array}$} & $\begin{array}{c}30-40 \text { microns } \\
\sigma_{\mu}=270 \mathrm{psi}\end{array}$ & $\begin{array}{c}y=0.92 x \\
-6.99\end{array}$ & 0.92 & $1837 \mathrm{psi}$ & 0.93 \\
\hline & $\begin{array}{c}40-50 \text { microns, } \\
\sigma_{\mu}=300 \mathrm{psi}\end{array}$ & $\begin{array}{c}y=0.87 x \\
-5.99\end{array}$ & 0.87 & $978 \mathrm{psi}$ & 0.97 \\
\hline \multirow{2}{*}{$\begin{array}{l}\text { PWHGMs } \\
\text { Using Bratt } \\
\text { et al }\end{array}$} & $\begin{array}{c}\text { 30-40 micron } \\
\text { (35 micron) }\end{array}$ & $\begin{array}{c}y=1.49 x \\
-17.27\end{array}$ & 1.49 & $108078 \mathrm{psi}$ & 0.95 \\
\hline & $\begin{array}{l}40-50 \text { micron } \\
\text { (45 micron) }\end{array}$ & $\begin{array}{c}y=1.46 x \\
-16.67\end{array}$ & 1.46 & 90927 psi & 0.85 \\
\hline $\begin{array}{c}3 \mathrm{M} \\
\text { S38HS }\end{array}$ & $\begin{array}{c}\text { 85-95 microns } \\
\text { (90 micron) }\end{array}$ & $\begin{array}{c}y=3.11 x \\
-28.43\end{array}$ & 3.11 & 9335 psi & 0.97 \\
\hline $\begin{array}{c}3 \mathrm{M} \\
\text { S60HS }\end{array}$ & $\begin{array}{l}\text { 60-70 microns } \\
\text { (65 micron) }\end{array}$ & $\begin{array}{c}y=1.60 x \\
-15.00\end{array}$ & 1.6 & $11790 \mathrm{psi}$ & 0.96 \\
\hline
\end{tabular}


As seen in Table 7, there is a decrease in strength as the microsphere diameter increases. The change in strength depends on the microsphere series. For the ARC HGMs, microspheres were $20-25 \%$ weaker than those one diameter range smaller. For PWHGMs, the strength of the 40-50 micron microspheres was approximately $65 \%$ of the value of the $30-40$ micron microspheres. Also note that in general the Weibull modulus was similar across all data sets.

The heat treated HGMs were the exception to the characteristic strength to diameter relationship as the strength increased from the 30-40 micron microspheres to the 45-55 micron microspheres. This was followed by a large decrease in the strength when comparing the 45-55 micron microsphere to the $60-70$ micron microspheres. The $45-55$ micron diameter range has the smallest Weibull modulus value of any two parameter analysis. When considering how different the heat treated HGMs are to the other stages of PWHGM production, it is likely that additional testing is required to increase the accuracy of their analyses. Due to the fact that there are multiple competing factors present during heat treatment, testing other types of heat treatments will be required to better understand the effects that heat treating has on the microspheres.

Comparing ARC HGMs to PWHGMs shows the strength decreases $75-80 \%$ from the initial stage of production to the PWHGM. The reason for this decrease is primarily due to the addition of porosity. The presence of porosity decreases the effective cross section carrying the load and consequently should increase the calculated failure stress. Porosity causing a change in the effective load carrying cross section is not enough to explain the difference in strength between ARC HGMs and PWHGMs. Although the percent porosity for the PWHGMs is not known, a reasonable value appears to be about $28 \%$ based on the porosity of similar porous glass [56]. Based on this value, the strengths for the two PWHGMs given in Table $7\left(1.444 \times 10^{3}\right.$ and 
$2.207 \times 10^{3} \mathrm{psi}$ ) should be $2.005 \times 10^{3}$ and $3.065 \times 10^{3} \mathrm{psi}$, respectively. When compared to the ARC HGMs $\left(6.894 \times 10^{3}\right.$ to $\left.9.072 \times 10^{3} \mathrm{psi}\right)$ there is still a large difference in strengths. There are additional effects caused by porosity that may explain the difference in strength. First, porosity will change the stress distribution throughout the wall. How the stresses are redistributed will depend on the porosity morphology. Second, any pores that contain cracks may contribute to the crack length, decreasing the amount of stress required to cause failure as shown in Equation (11).

Equation (20) was used to combine the data from different sets into one larger data set. Each stage of PWHGM production had its data normalized to that of the 35 micron data. This microsphere size was chosen as it was the average diameter of the 30-40 micron range. The results for these combined analyses are shown in Figure 43 and Table 7. With the exception of the ARC HGMs, the combined analyses were very close to their 30-40 micron range values. The ARC HGMs had a combined Weibull modulus and characteristic strength that were slightly higher than that of the data sets they were created from.

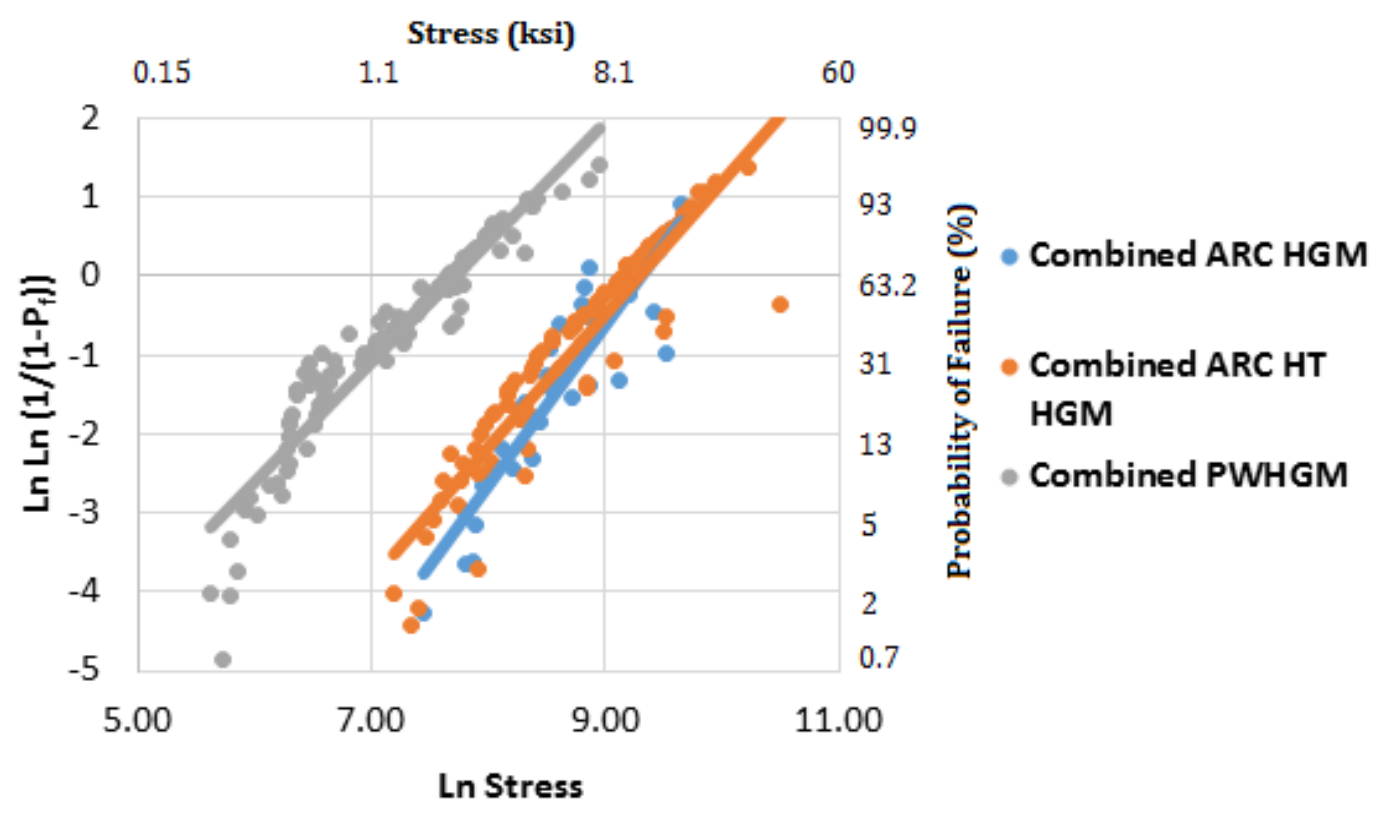

Figure 43: Effects of Different Stages of Production on Combined Data Sets 
The Weibull analysis for the combined 30-40 micron PWHGMs was used to predict the probability of failure for PWHGMs at a 70 micron diameter and 15 micron diameter as seen in Figure 44. This application of the Weibull analysis illustrates one of its predictive capabilities. That is, if one has completed an analysis for a specific microsphere size, it is possible to predict the strength and probability of failure for other microspheres sizes without additional testing.

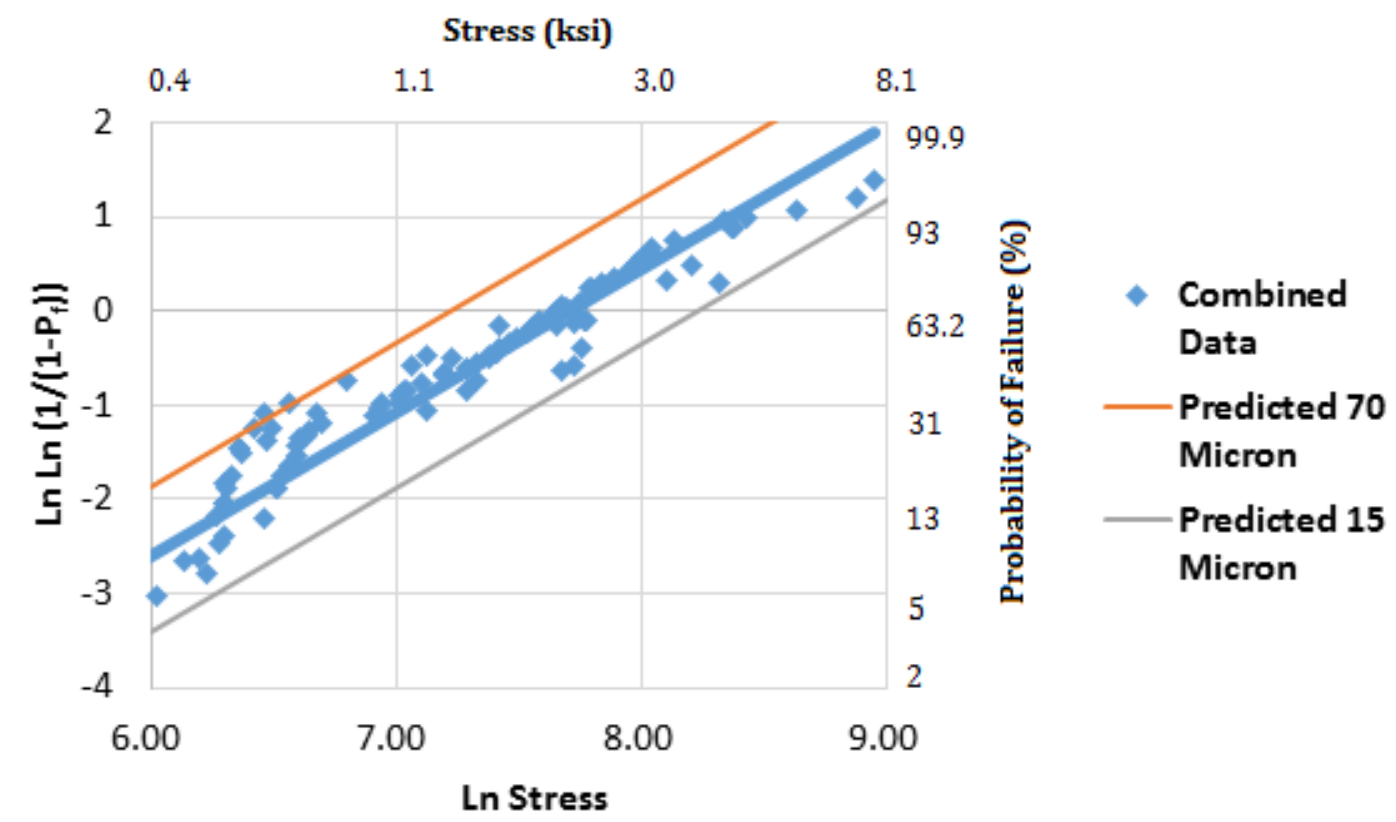

Figure 44: 35 Micron PWHGM Data Used to Predict Strength of PWHGMs at 70 Microns and 15 Microns

While the majority of the data in this research was evaluated using the two parameter Weibull analysis, a set of three parameter analyses were performed on the PWHGMs in order to determine the effects of the threshold parameter, $\sigma_{\mu}$. In these analyses the threshold parameter was set to a few psi smaller than the lowest calculated stress. For the 30-40 micron range this was 270 psi and for the 40-50 micron range this was 300 psi. The Weibull moduli and characteristic strength for these analyses were $\sim 0.7$ and $\sim 400$ psi smaller than the two parameter analyses. Comparing the $r^{2}$ values for the two and three parameter analyses shows that the three parameter analysis fit the data better for the 40-50 micron microspheres and worse for the 30-40 
micron microspheres. Depending on the value used for the threshold parameter, the three parameter analyses should have a better fitting linear regression than two parameter analyses. Assuming that the threshold is not equal to zero is also more realistic as this means at least some minimal stress must be applied to cause failure [57].

In addition to the Weibull analyses conducted using stress calculated from Equation (21), a second set of two parameter Weibull analyses was conducted on the PWHGMs with stress calculated using Equation (7) from Bratt et al. The results from these analyses showed that the crush strength using Equation (7) was 50 to 60 times higher than those calculated from Equation (21) and the Weibull moduli were very similar between both analyses. Using Bratt et al's equation is questionable considering that it does not take into account the diameter of the microsphere.

Other investigators have examined the strength of glass using the Weibull analysis. Two Weibull analyses were used to compare the results seen in other research to those found in this thesis. One was a 3 point bend testing on $20 \mathrm{~mm}$ long borosilicate glass bars by Deilhof et al [58] and the second was tensile testing on 20mm long, 15 micron diameter, Silane coated E-glass fibers by Feih et al [59]. Deilhof et al reported values for the Weibull modulus and characteristic strength to be 5.0 and 35,186 psi for borosilicate glass. Feih et al found that E-glass fibers had a Weibull modulus and characteristic strength of 4.62 and 243,663 psi. Neither of these analyses applied the same type of testing used in this research. The characteristic strengths and Weibull Moduli from both investigators were higher than any value obtained from the microspheres based on Equation (22). Caution must be used when making these types of comparisons due to the difference in test methods and types of samples. A better comparison between the two analyses could be made if the load factor, k, used in Equations (18) and (20) was known. 
Compared to the tensile test and 3 point bend test used by the other investigators, the stress distribution on the microspheres under uniaxial compression testing is more complex making it difficult to determine $\mathrm{k}$ and compute an accurate stress at failure. In order to better understand the stress distribution acting on the microspheres under uniaxial compression finite element analysis will probably be required.

The characteristic strength of the 3M S60HS microspheres was approximately 1.8 times stronger than the ARC HGMs of the same diameter. At 85-95 microns, the S38HS microspheres have a characteristic strength close to ARC HGMs in the 45-55 micron diameter range. Using Equation (20) to normalize the 3M S8HS microspheres to 35 microns shows that they should have a characteristic strength of $15,850 \mathrm{psi}$ and a Weibull modulus of 3.05. These values are higher than any ARC microsphere in the 30-40 micron range.

The critical crack length (i.e. the crack length causing failure) for the characteristic strength was calculated using Equation (3) with the $A$ parameter assumed to be 1 for all samples. The elastic modulus and surface energy of borosilicate glass were assumed to be $64 \mathrm{GPa}$ [60] and $0.11 \mathrm{~J} / \mathrm{m}^{2}$ [61], respectively. For silica glass elastic modulus and surface energy were assumed to be $73 \mathrm{GPa}$ [12] and $0.31 \mathrm{~J} / \mathrm{m}^{2}$ [62], respectively. The crack length results for all microspheres calculated using Equation (22) were unrealistically high and larger than the microsphere wall. In the PWHGMs and 30-40 micron heat treated HGMS the calculated critical crack lengths were extremely high with all values being larger than the microspheres tested (crack length $>100$ microns). With the other microspheres tested, the crack lengths were between one to five microns which is larger than the average wall thickness of the microspheres. When using Bratt et al's equation, the crack lengths are closer to expectations with lengths between four to six nanometers. This could indicate that the values produced by using Equation 
(10) are closer to the actual strengths of the microspheres or it could indicate that the $A$ value used was incorrect. It may also indicate that the cracks in PWHGMs are connected to pores spread throughout that wall, which would partly explain the extremely large value seen.

Finally, it is should be understood that the accuracy of the Weibull analysis is dependent on the number of samples tested. With the 10 data points used for most of the analyses, the accuracy of the Weibull modulus is close to $+/-40 \%$. For the PWHGMs and the 30-40 micron diameter heat treated ARC HGMs, the Weibull modulus accuracy is close to $+/-10 \%$ because 50 data points were used in each analysis. Additional testing is required to increase the overall accuracy of the results. 


\section{Ch. 7: Summary}

Porous wall hollow glass microspheres are a unique and important discovery in materials technology. Due to their interconnecting porosity, size, and material composition, PWHGMs can be used in many filtration and encapsulation applications. While many manufacturers of microspheres do bulk crush strength testing, it is possible to collect more detailed and representative results by testing individual microspheres using Nanoindentation equipment. Using a flat end tip it is possible to crush a single microsphere to gain data on the crush strength. For this research, the crush strength was calculated based on the load required to fracture the microsphere divided by the solid portion of the cross sectional area at the equator. While this may not be the most quantitative method of calculating the crush strength, it does provide a semi quantitative analysis since it uses both the microsphere diameter and wall thickness in the calculations. These are important factors in the strength as they directly affect the volume and determine the number of critical flaws in a system.

Due to the way brittle materials (microspheres) fracture there will be scattering in the data meaning multiple tests must be run to better understand the results. The Weibull analysis provides an excellent method for statistically analyzing this type of data as it takes into account the effects of volume on the strength of the material. With this analysis it is possible to determine the characteristic strength and Weibull modulus for microspheres with similar diameters. This data can be used to predict strengths for microspheres with other diameters.

By using nanoindentation and the Weibull analysis, it was possible to analyze how the various stages of production and different sphere diameters affect the crush strength of PWHGMs. Calculating the crush strength proved to be a challenge as stress distribution on a hollow sphere between two plates is extremely complex. Currently there is no universally 
agreed upon theory and there may not be a closed form solution to the equations used to define this system.

It was shown in the two parameter Weibull results that the microsphere diameter and crush strength had an inverse relationship. It was also shown that the strength of the PWHGM decreases from the beginning of production to the final product. The only outlier in these results were the heat treated HGMs. This stage of production saw an increase in strength when going from the 30-40 micron microsphere to the 45-55 micron microspheres. There was a sharp decrease in strength when comparing the 45-55 micron microspheres to the 60-70 micron microspheres. Testing microspheres heat treated under different conditions is recommended to better understand the effects at play during heat treatment.

The Weibull modulus, which represents scattering in the data, was similar across all stages of PWHGM production for a two parameter analysis. In addition to the two parameter analyses, three parameter analyses were conducted on the PWHGMs. These analyses used threshold parameters a few psi smaller than the lowest calculated strength values. This caused the results to stretch out and created more scattering in the data. The values of the Weibull moduli and characteristic strength were also lower than the two parameter results.

When compared to different types of strength tests on similar materials performed by other investigators the strength of the microspheres was extremely low. This comparison is not completely accurate without using the load factor to make the stresses from each test comparable. Compared to 3M microspheres, the strength of the PWHGMs was also much lower. This is not unexpected when considering how the wall porosity will have a negative impact on the strength of the microspheres. 
Crack length calculations for the characteristic strength also shows that the strength values used were lower than expected. All of the crack lengths calculated using Equation (21) were larger than the average wall thickness of the microspheres. The PWHGMs were extremely large with crack lengths greater than the diameter of the PWHGMs used. Bratt et al's method produced crack lengths on the nanometer scale which compared more favorably with the microsphere wall thickness. However Bratt et al's equation for fracture stress is questionable and it is more likely that the pores inside the microspheres are playing a role in the fracture strength.

In addition to the analysis of the crush strength of the microspheres, a comparative analysis of the microsphere size distribution was performed. The purpose of this analysis was to determine how well the results of the microscope measurements performed at ARC compared to laser light scattering performed at Virginia Tech. The results indicated that both tests presented essentially the same microsphere size distributions. Having confidence in the microsphere diameter was necessary since this variable has a major effect on the Weibull analysis.

There are many possible pathways for future research now that it has been shown that analyzing the mechanical properties of microspheres is possible through nanoindentation. First, additional testing is recommended to improve the accuracy of the current data sets. This could be expanded to a study on the effects heat treatment has on microspheres by testing microspheres subjected to different heat treatments. Second, additional work to properly analyze the data when calculating the crush strength is needed. Because it may not be possible to create an equation to accurately calculate the crush strength it is recommended that finite element analysis be used to further interpret the data. Third, in addition to crush testing nanoindentation could be used to analyze other properties of microspheres. The NanoTest instrument is able to create tests 
that would examine the creep, cyclic loading, and impact properties of individual microspheres. With additional attachments it would be possible to examine the properties of microspheres in various environments such as high humidity. These are a few suggestions for research that would provide additional understanding of the strengths of microspheres. 


\section{Appendices}

\section{Appendix A: Light Scattering Results}

Figure 45 through Figure 50 display the combined results for each of the microsphere series tested. The microsphere diameter is the $\mathrm{x}$ axis on the bottom of the graph. The $\mathrm{y}$-axis on the right is the percent undersize that corresponds to the cumulative distribution function. This axis is used to determine what percentage of the microspheres are under a certain size. The yaxis on the left, $\mathrm{q}(\%)$, corresponds to the probability distribution function and indicates what percentage of the total distribution the microspheres at a specified diameter comprise. Most graphs show a bimodal distribution where one part is in the submicron range. The likely cause for the readings in the submicron range is the debris on the microspheres. As seen in Figure 25, the microspheres are covered in what may be carbon. ARC has shown that this debris will come off if the microspheres are washed. The particles in the submicron range are most likely the debris washing off of the microspheres while in the instrument.

There is one other anomaly found in some of the data. Some of the tests produced results where there were microspheres in the 100 to 1000 micron range. These results are not reported in any table provided by $3 \mathrm{M}$ and were not seen in ARC's analyses. They do not consistently appear, so one conclusion is that some microspheres did not disperse correctly before testing started and formed agglomerates that produce the anomalies in the data. In the first group of tests run on the S38HS microspheres, agglomeration problems were enough to warrant a second set of tests to be performed. For the second group, the anomaly was minimal compared to the original. Upon examining the main part of each distribution, the data seems to confirm the information provided by the $3 \mathrm{M}$ information sheet. 

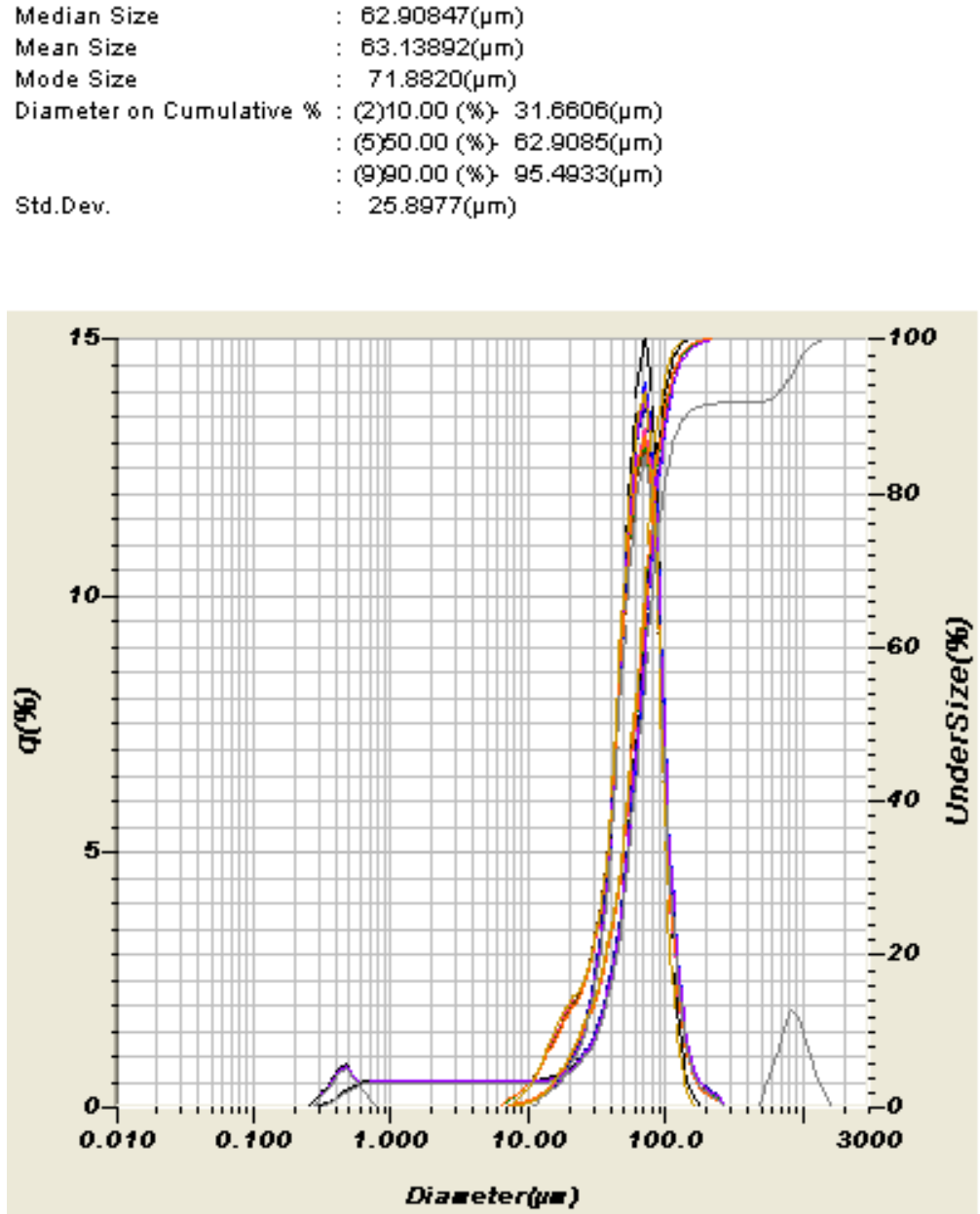

Figure 45: ARC HGM Laser Scattering Results Showing 5 Samples from the Same Batch with Each Sample Run Twice 

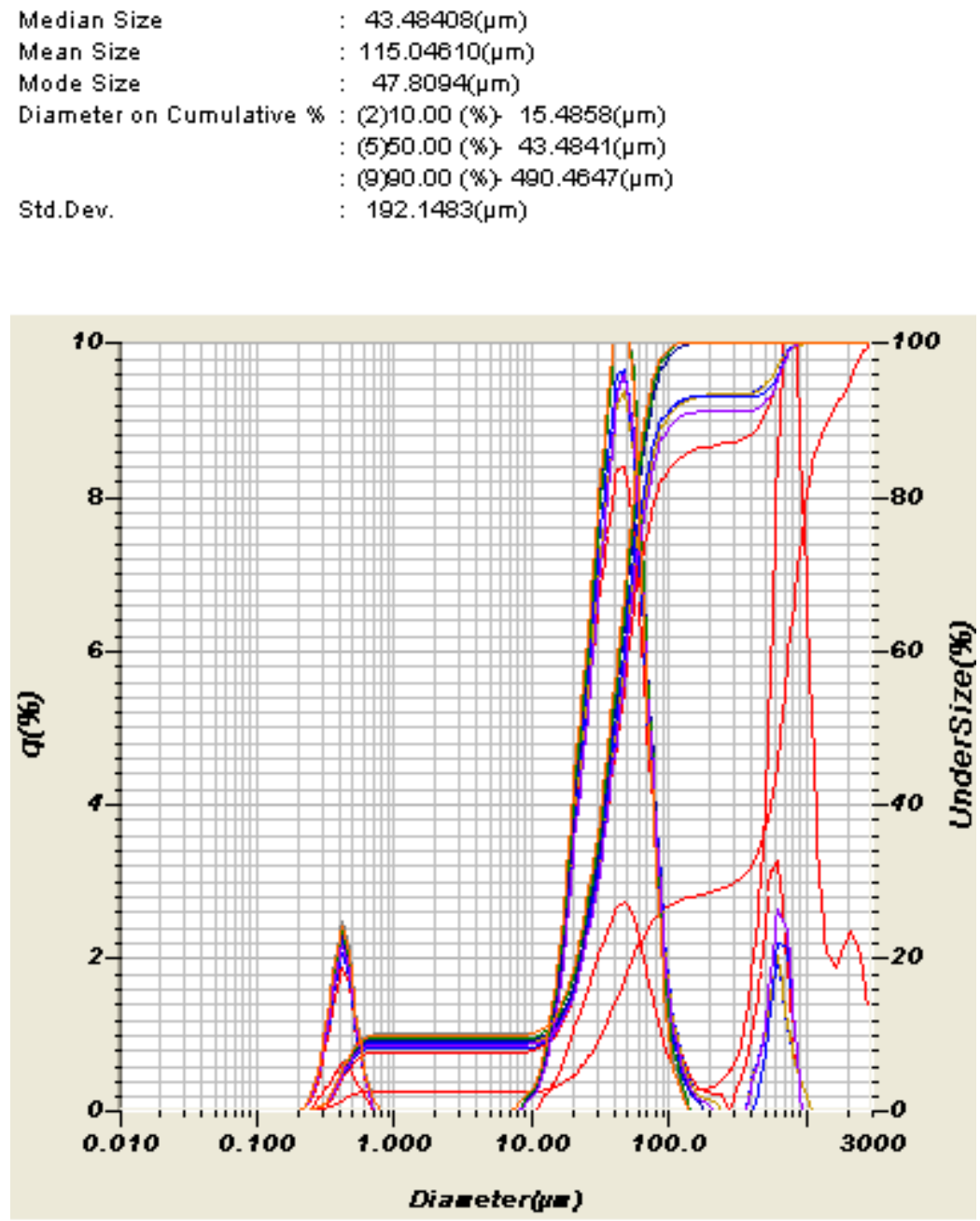

Figure 46: S38HS Test 1-5 Laser Scattering Results Showing 5 Samples from the Same Batch with Each Sample Run Twice 

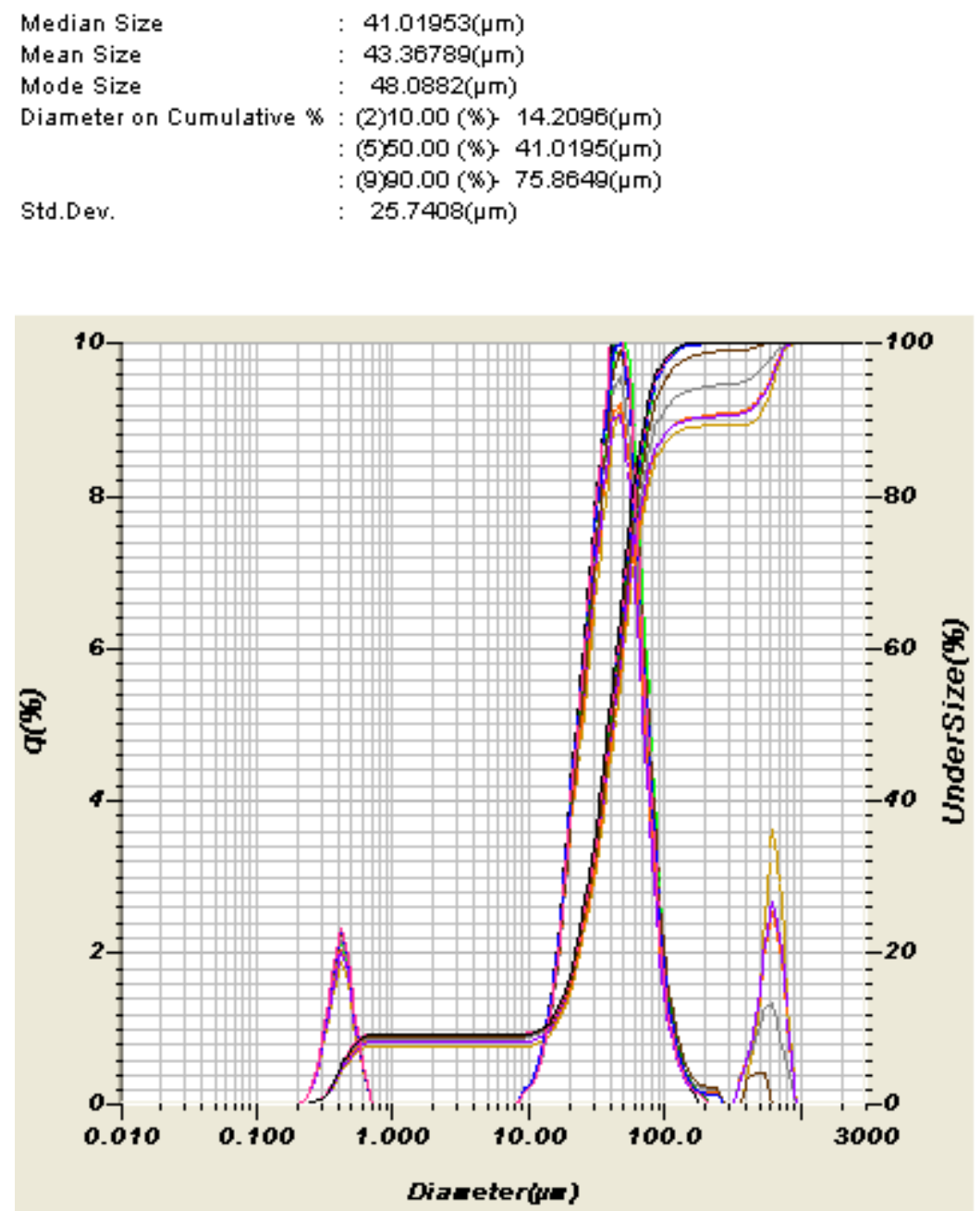

Figure 47: S38HS Test 6-10 Laser Scattering Results Showing 5 Samples from the Same Batch with Each Sample Run Twice 

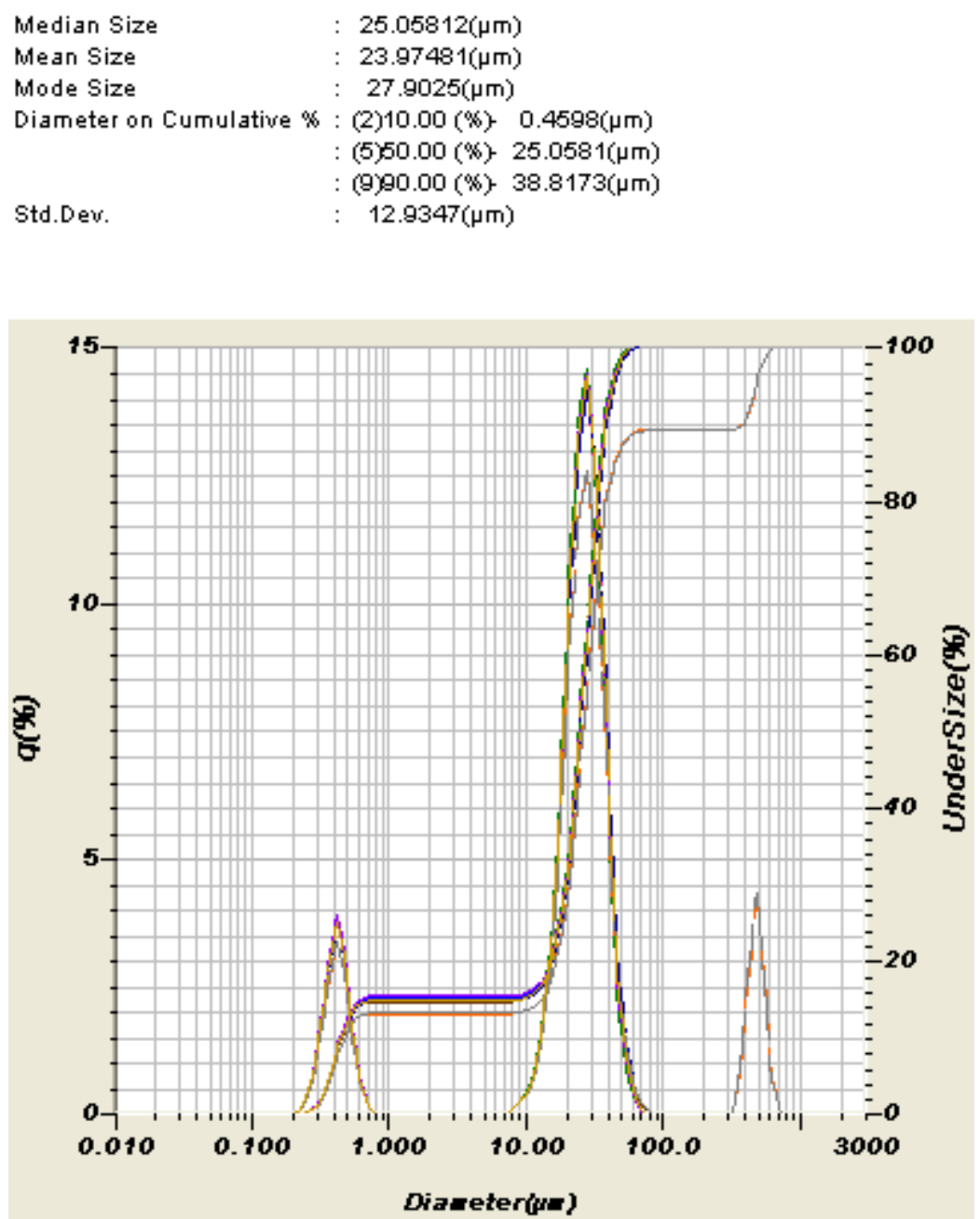

Figure 48: S60HS Laser Scattering Results Showing 5 Samples from the Same Batch with Each Sample Run Twice 

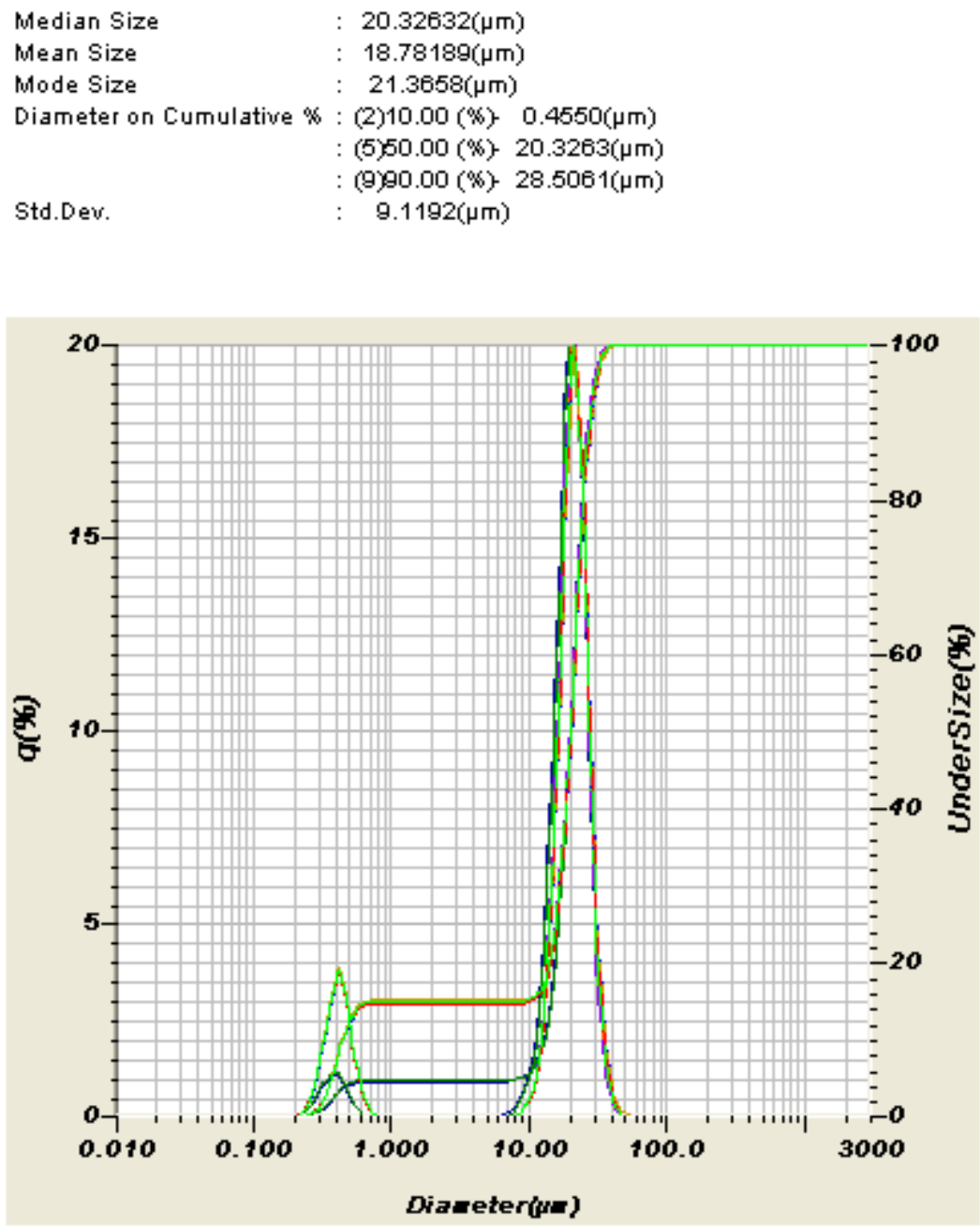

Figure 49: IM16K Laser Scattering Results Showing 5 Samples from the Same Batch with Each Sample Run Twice 

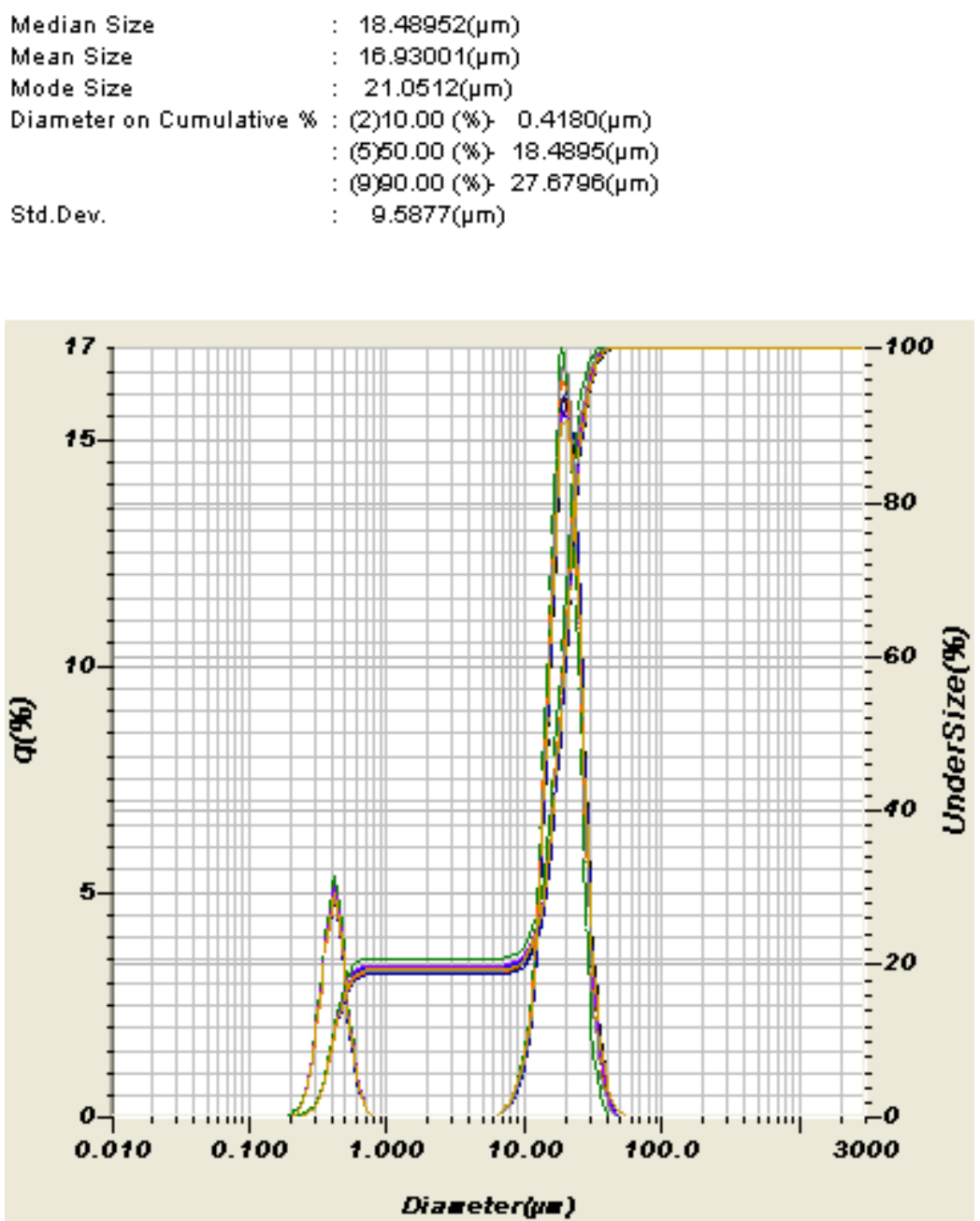

Figure 50: IM30K Laser Scattering Results Showing 5 Samples from the Same Batch with Each Sample Run Twice 


\section{Appendix B: ARC Microscope Analyses and 3M Data}

Each type of microsphere had at least one sample used in the microscopy analyses. Ten micrographs were recorded for each sample in random sections to prevent favoring of any type of distribution. Each image was then analyzed with measuring programs and statistical analyses tools in the software provided by Axiovision for the Zeiss Optical Microscope. Excel was used to combine the data tables from each micrograph as Axiovision provides data that is compatible.

Table 8 through Table 14 show the statistical results for the combined data of each type of microsphere, including minimum, maximum and mean glass microsphere diameters along with more detailed size distributions as well as other information. In Table 8, another comparison is provided between published glass microsphere sizes by $3 \mathrm{M}$ (measured using a different technique) with the experimentally derived data obtained at ARC on the same types of microspheres. The data published by $3 \mathrm{M}$ only contains volume distributions, not number distributions, so to compare the 3M's published distributions, all data on microsphere diameters were converted to volume, including ARC's experimental distributions. In Table 9, a comparison of the numeric distributions as measured in the lab for the ARC HGMs and the $3 \mathrm{M}$ microspheres is summarized. Table 10 provides data on ARC's nonleached HGMs, while Table 11 through Table 14 provide analyses for each of the four types of $3 \mathrm{M}$ commercial microspheres. The data in these figures show the raw output of the analysis edited to two decimal places to provide a more realistic value. 
Table 8: Published vs. ARC Measured Distributions for 3M Microspheres [24]

\begin{tabular}{|c|c|c|c|c|c|c|}
\hline & \multicolumn{5}{|c|}{} & $\begin{array}{c}\text { Apparent } \\
\text { Density } \\
\text { Microsphere Type }\end{array}$ \\
& $10 \%$ & $50 \%$ & $90 \%$ & $\begin{array}{c}\text { Max Size } \\
\text { (g/cc }\end{array}$ & $\begin{array}{c}\text { Strength } \\
(\mathrm{psi})\end{array}$ \\
\hline & $15 \mu \mathrm{m}$ & $40 \mu \mathrm{m}$ & $75 \mu \mathrm{m}$ & $85 \mu \mathrm{m}$ & 0.38 & 5,500 \\
\hline S38HS (3M) & $18 \mu \mathrm{m}$ & $53 \mu \mathrm{m}$ & $61 \mu \mathrm{m}$ & - & - & - \\
\hline S38HS (ARC) & $11 \mu \mathrm{m}$ & $30 \mu \mathrm{m}$ & $50 \mu \mathrm{m}$ & $60 \mu \mathrm{m}$ & 0.60 & 18,000 \\
\hline S60HS (3M) & 16 & - & - & - \\
\hline S60HS (ARC) & $16 \mu \mathrm{m}$ & $30 \mu \mathrm{m}$ & $44 \mu \mathrm{m}$ & - & 0.46 & 16,000 \\
\hline IM16K (3M) & $12 \mu \mathrm{m}$ & $20 \mu \mathrm{m}$ & $30 \mu \mathrm{m}$ & $40 \mu \mathrm{m}$ & - & - \\
\hline IM16K (ARC) & $13 \mu \mathrm{m}$ & $20 \mu \mathrm{m}$ & $29 \mu \mathrm{m}$ & - & 0.60 & 28,000 \\
\hline IM30K (3M) & $9 \mu \mathrm{m}$ & $16 \mu \mathrm{m}$ & $25 \mu \mathrm{m}$ & $29 \mu \mathrm{m}$ & - & - \\
\hline IM30K (ARC) & $12 \mu \mathrm{m}$ & $21 \mu \mathrm{m}$ & $31 \mu \mathrm{m}$ & - & - & \\
\hline
\end{tabular}

Table 9: Numeric Distribution for 3M and ARC Microspheres

\begin{tabular}{|l|c|c|c|}
\hline Microsphere & \multicolumn{3}{|c|}{ Numeric Distribution } \\
\hline & $10 \%$ & $50 \%$ & $90 \%$ \\
\hline ARC HGMs & $20 \mu \mathrm{m}$ & $37 \mu \mathrm{m}$ & $70 \mu \mathrm{m}$ \\
\hline S38HS (3M) & $9 \mu \mathrm{m}$ & $16 \mu \mathrm{m}$ & $32 \mu \mathrm{m}$ \\
\hline S60HS (3M) & $9 \mu \mathrm{m}$ & $14 \mu \mathrm{m}$ & $28 \mu \mathrm{m}$ \\
\hline IM16K (3M) & $10 \mu \mathrm{m}$ & $15 \mu \mathrm{m}$ & $22 \mu \mathrm{m}$ \\
\hline IM30K (3M) & $9 \mu \mathrm{m}$ & $14 \mu \mathrm{m}$ & $23 \mu \mathrm{m}$ \\
\hline
\end{tabular}


Table 10: ARC HGM Combined Table

\begin{tabular}{|c|c|c|}
\hline Description & Number & Volume \\
\hline Count & 1492 & - \\
\hline $\begin{array}{c}\text { Microsphere } \\
\text { Diameter }\end{array}$ & $\mu \mathrm{m}$ & $\mu \mathrm{m}$ \\
\hline Minimum & 3 & - \\
\hline Maximum & 207 & - \\
\hline Mean & 42 & - \\
\hline 25-Quartile & 27 & - \\
\hline $\begin{array}{c}\text { 50-Quartile } \\
\text { (Median) }\end{array}$ & 37 & 70 \\
\hline 75-Quartile & 54 & - \\
\hline 10-Percentile & 20 & 40 \\
\hline 90-Percentile & 70 & 103 \\
\hline Skewness & 1.34 & - \\
\hline
\end{tabular}

Table 11: S38HS Combined Table

\begin{tabular}{|c|c|c|}
\hline Description & Number & Volume \\
\hline Count & 2873 & - \\
\hline $\begin{array}{c}\text { Microsphere } \\
\text { Diameter }\end{array}$ & $\mu \mathrm{m}$ & $\mu \mathrm{m}$ \\
\hline Minimum & 5 & - \\
\hline Maximum & 112 & - \\
\hline Mean & 18 & - \\
\hline 25-Quartile & 11 & - \\
\hline $\begin{array}{c}\text { 50-Quartile } \\
\text { (Median) }\end{array}$ & 14 & 53 \\
\hline 75-Quartile & 22 & - \\
\hline 10-Percentile & 8 & 18 \\
\hline 90-Percentile & 32 & 61 \\
\hline Skewness & 1.86 & - \\
\hline
\end{tabular}


Table 12: S60HS Combined Table

\begin{tabular}{|c|c|c|}
\hline Description & Number & Volume \\
\hline Count & 751 & - \\
\hline $\begin{array}{c}\text { Microsphere } \\
\text { Diameter }\end{array}$ & $\mu \mathrm{m}$ & $\mu \mathrm{m}$ \\
\hline Minimum & 5 & - \\
\hline Maximum & 59 & - \\
\hline Mean & 16 & - \\
\hline 25-Quartile & 10 & - \\
\hline $\begin{array}{c}\text { 50-Quartile } \\
\text { (Median) }\end{array}$ & 14 & 30 \\
\hline 75-Quartile & 20 & \\
\hline 10-Percentile & 9 & 16 \\
\hline 90-Percentile & 28 & 44 \\
\hline Skewness & 1.47 & - \\
\hline
\end{tabular}

Table 13: IM16K Combined Table

\begin{tabular}{|c|c|c|}
\hline Description & Number & Volume \\
\hline Count & 1177 & - \\
\hline $\begin{array}{c}\text { Microsphere } \\
\text { Diameter }\end{array}$ & $\mu \mathrm{m}$ & $\mu \mathrm{m}$ \\
\hline Minimum & 5 & - \\
\hline Maximum & 40 & - \\
\hline Mean & 15 & - \\
\hline 25-Quartile & 11 & - \\
\hline $\begin{array}{c}\text { 50-Quartile } \\
\text { (Median) }\end{array}$ & 14 & 20 \\
\hline 75-Quartile & 18 & - \\
\hline 10-Percentile & 10 & 13 \\
\hline 90-Percentile & 22 & 30 \\
\hline Skewness & 1.00 & - \\
\hline
\end{tabular}


Table 14: IM30K Combined Table

\begin{tabular}{|c|c|c|}
\hline Description & Number & Volume \\
\hline Count & 1815 & - \\
\hline $\begin{array}{c}\text { Microsphere } \\
\text { Diameter }\end{array}$ & $\mu \mathrm{m}$ & $\mu \mathrm{m}$ \\
\hline Minimum & 5 & - \\
\hline Maximum & 44 & - \\
\hline Mean & 15 & - \\
\hline 25-Quartile & 11 & - \\
\hline $\begin{array}{c}\text { 50-Quartile } \\
\text { (Median) }\end{array}$ & 14 & 22 \\
\hline 75-Quartile & 18 & - \\
\hline 10-Percentile & 9 & 13 \\
\hline 90-Percentile & 23 & 31 \\
\hline Skewness & 1.04 & - \\
\hline
\end{tabular}

Comparing the volume distributions provided by $3 \mathrm{M}$ to the experimental distributions shows that the distributions are very similar. The largest differences were observed in the larger S38HS microspheres. In the S60HS and IM30K microspheres measured distribution values were generally around 5 microns different from published values while the IM16K microsphere values are less than a micron different from published values. This correlation is remarkable because $3 \mathrm{M}$ versus $\mathrm{ARC}$ measurements use different techniques and additional variations would be expected due to samples taken from various locations within the batch. 
Appendix C: Bar Graphs of the Numeric and Volumetric Fractions (\%) of Microspheres with Specified Diameters Using Data Obtained from ARC's Microscope

Appendix $\mathrm{C}$ takes the numeric and volumetric distribution data used to calculated the results shown in Table 10 through Table 14 plots them on bar graphs for a visual comparison. Figure 51 shows the results of the ARC HGMs while Figure 52 through Figure 55 shows the results for the $3 \mathrm{M}$ microspheres.

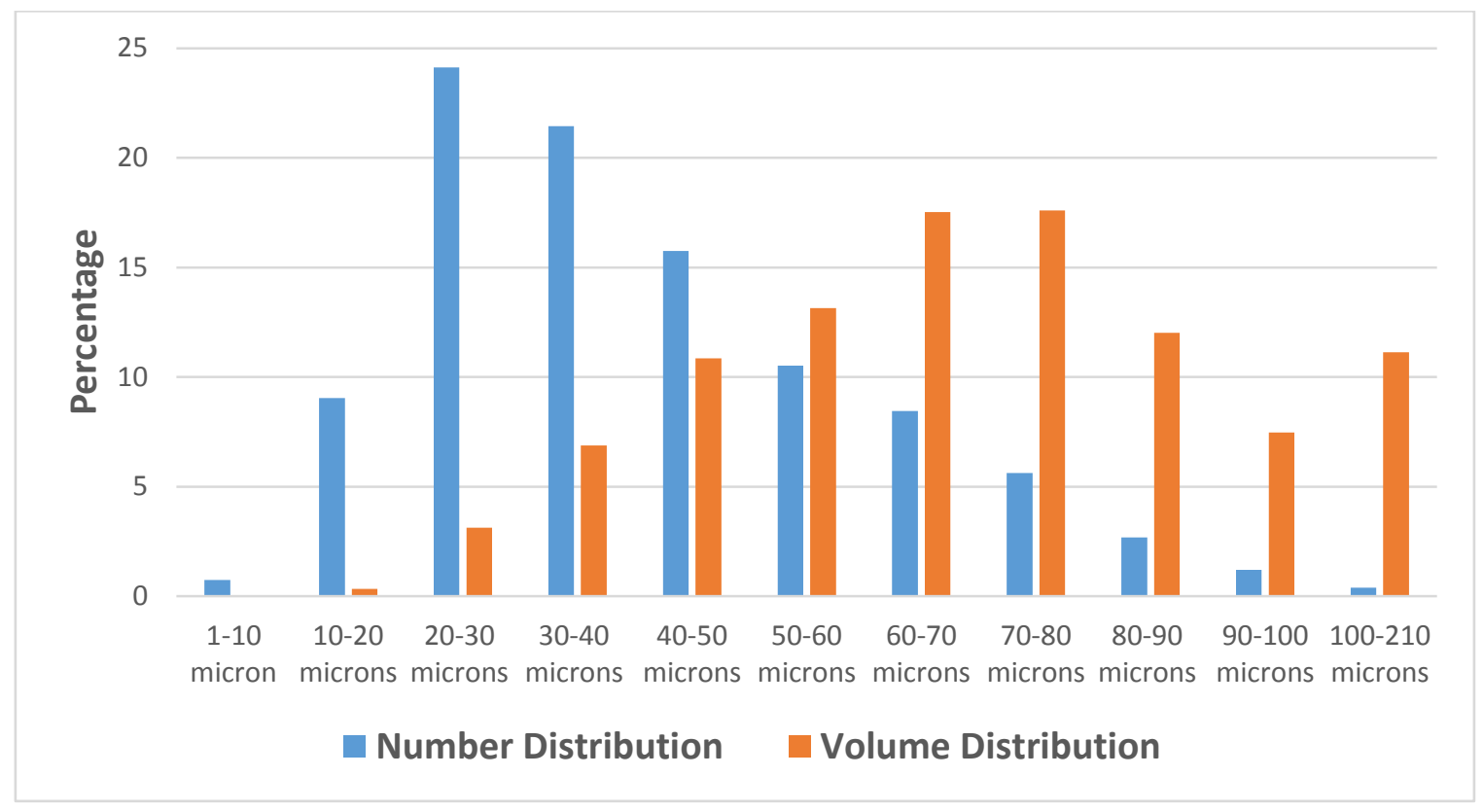

Figure 51: ARC HGM Bar Graph 


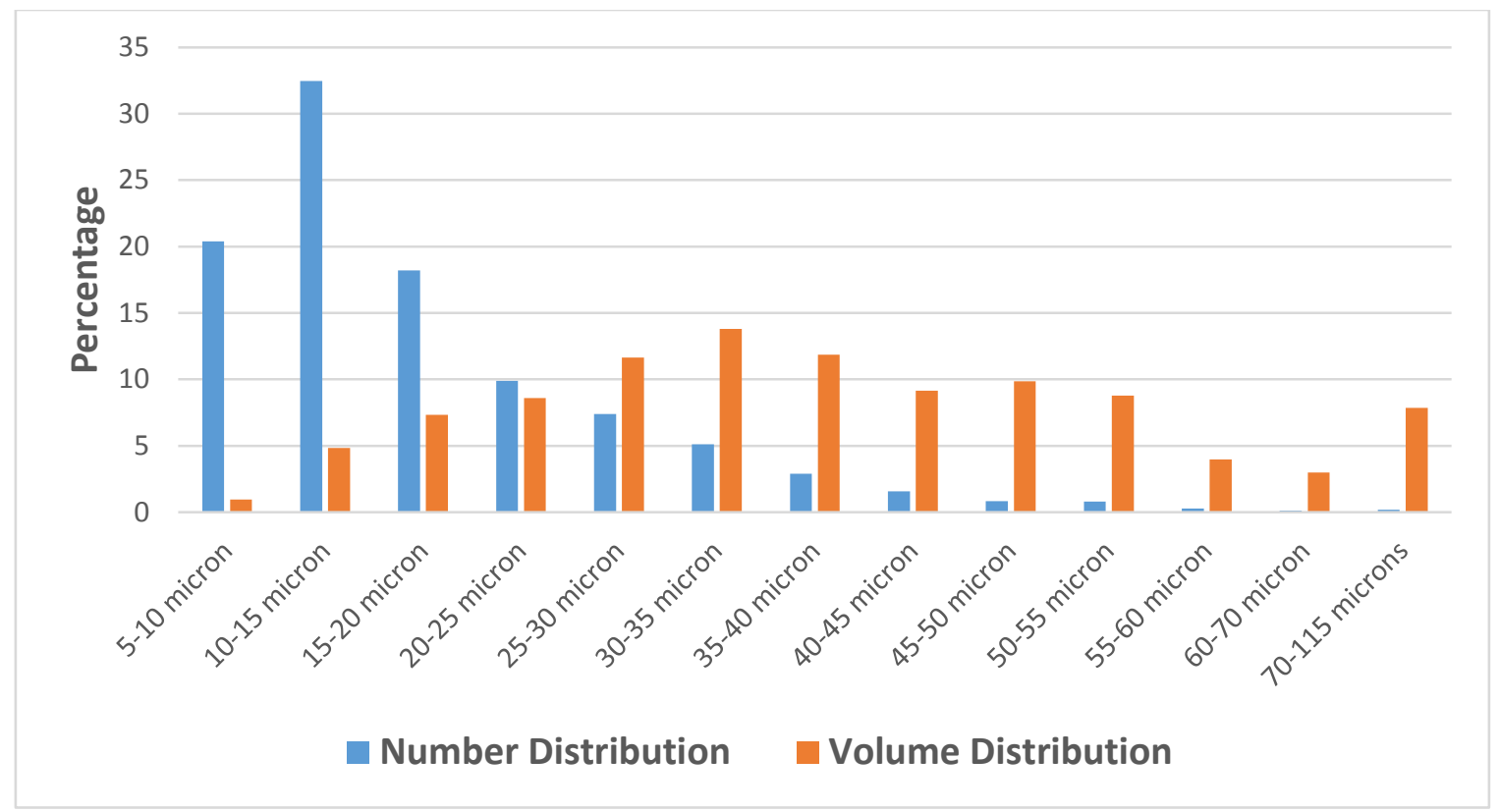

Figure 52: S38HS Bar Graph

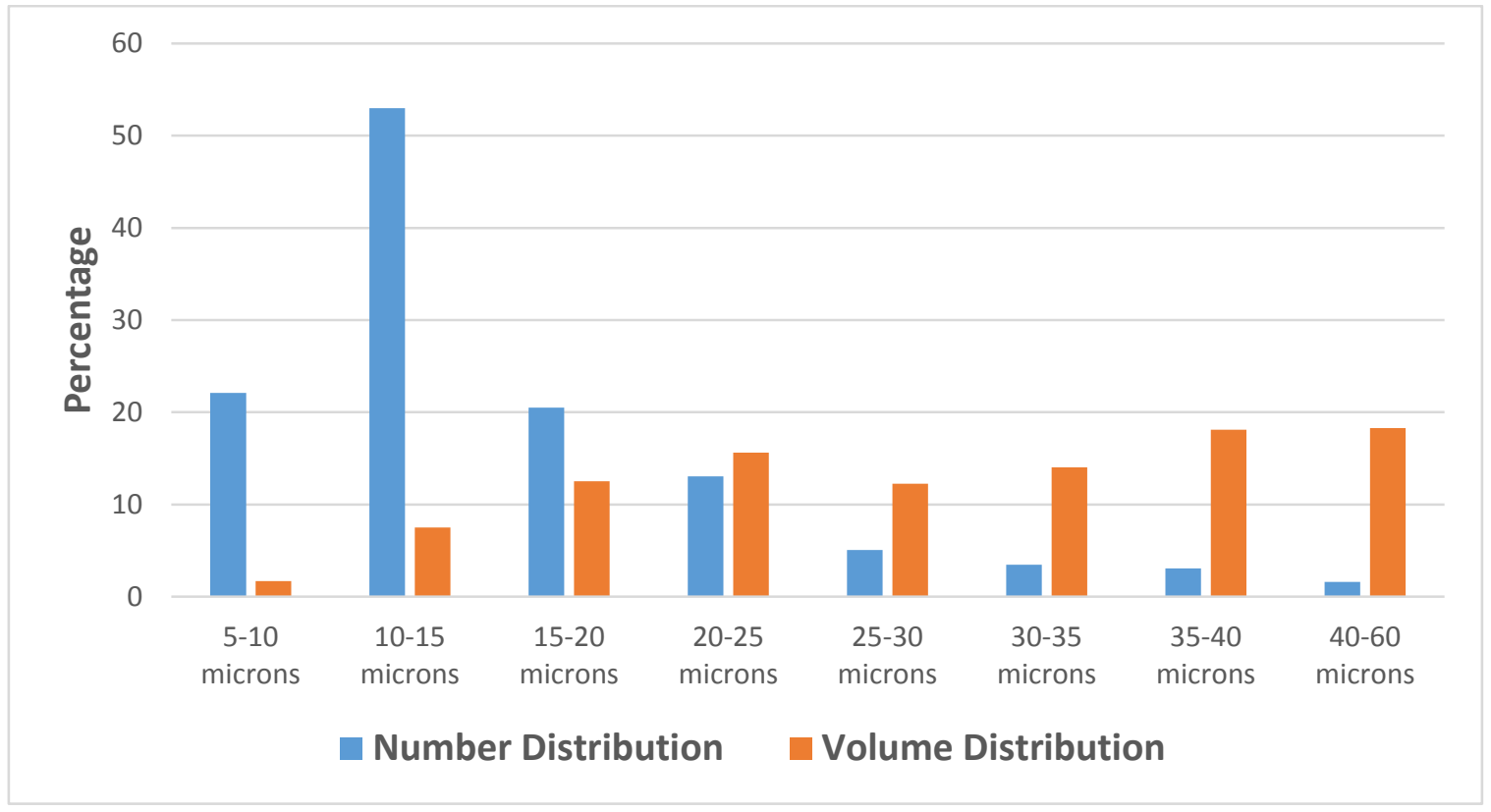

Figure 53: S60HS Bar Graph 


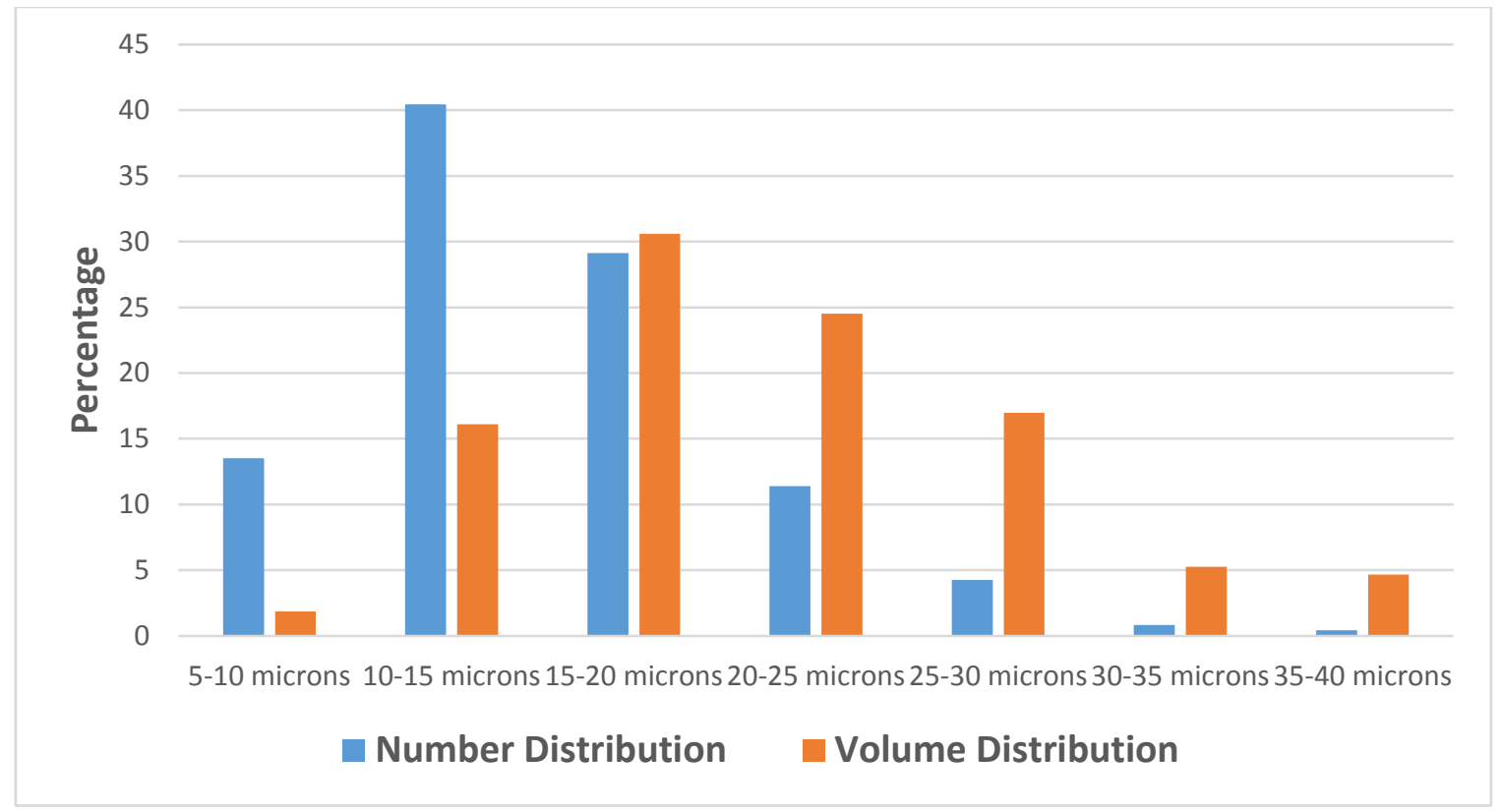

Figure 54: IM16K Bar Graph

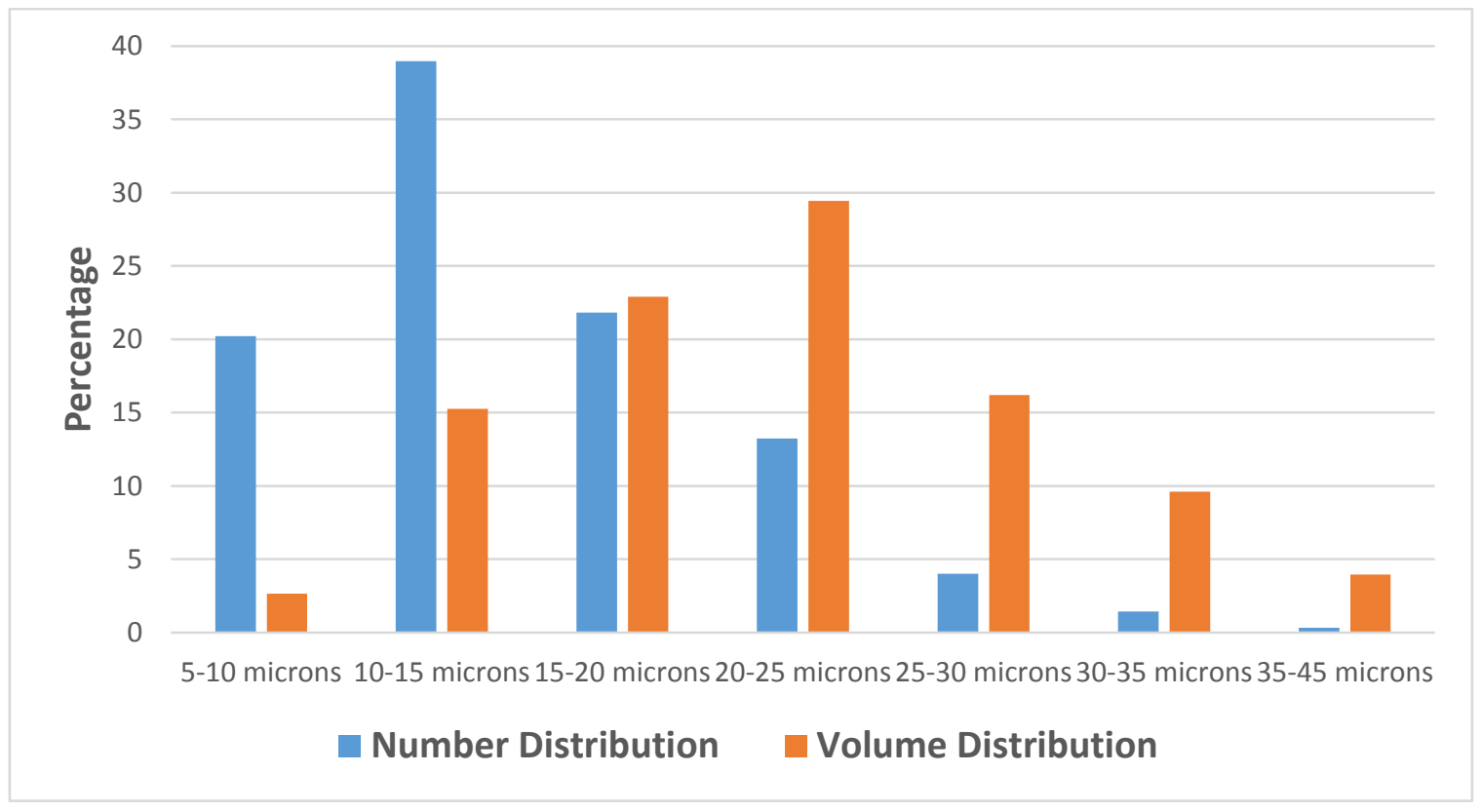

Figure 55: IM30K Bar Graph 


\section{Appendix D: Additional Variations to Nano Crush Test Results}

In this appendix additional variations seen in the nano crush test results are shown. Figure 57 shows a test where there is an initial increase in load before the nanoindenter tip moves. In this case it is probable that the microsphere is hard enough that the small load applied is not enough to cause any deformation. After the initial increase in load, the data output will resemble one of the other variations discussed in this section.

Figure 58 illustrates a situation where there are small aberrations in the initial region. The reason for these small aberrations is not understood at the present. Figure 59 is similar to Figure 58 except that the aberrations were much larger. In this case it was determined that the microsphere had failed at the first change in slope. Afterwards the tip hit another portion of the microsphere that required additional force to break. The fracture in this type of output was identified as the point where tip displacement increased without additional load for over one micron. This phenomenon was also seen by Carlisle et al who thought the response was created by compartments within the microsphere [32]. However, it is not clear that internal compartments were responsible for the results in the present work based on SEM results. In Figure 60 multiple changes in slope of the initial region are seen before fracture occurs. Unlike the results shown in Figure 59, the slope does not go flat in this region which seems to indicate that failure had not occurred yet. This result is similar to the "flawed single wall" microspheres seen by Carlisle et al and could be caused by surface and wall irregularities and flaws [32].

Figure 61 shows a test result that occurred in some microspheres only a few data points were recorded. This suggests that the tip speed was too fast in these particular tests and the load at which fracture occurs is not very clear. The initial region of the graph shows very little deformation occurring and the change between regions does not include a point where the slope 
clearly changes. The second region of the graph is different from most tests as the load required to increase tip displacement is not at or close to zero. Images taken of microspheres with these types of test results showed a crushed microsphere confirming that failure did occur as seen in Figure 56. There were only a few tests that resulted in this type of output and changes to the load rate during testing fixed the issue. Figure 62 shows an additional variation that occurred in some tests. When this result occurred the nanoindenter recorded data points as it removed the tip from the sample surface. It is unknown why this additional data was recorded when normal output would show only a decrease in load applied to the tip. This type of output did not seem to affect the test results or indicate anything wrong with the microsphere tip.

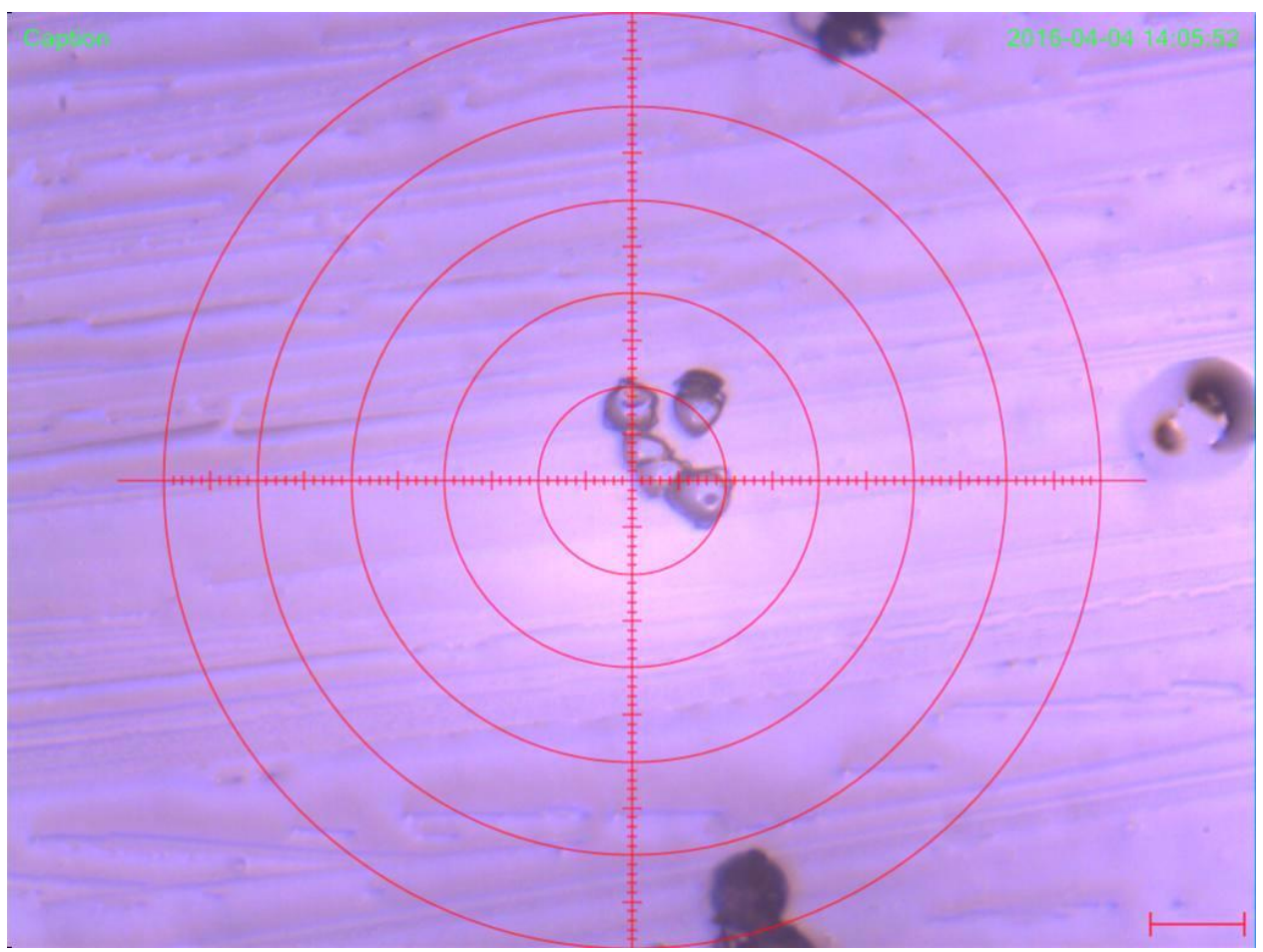

Figure 56: Microsphere after Test where Not Many Data Points were Recorded 


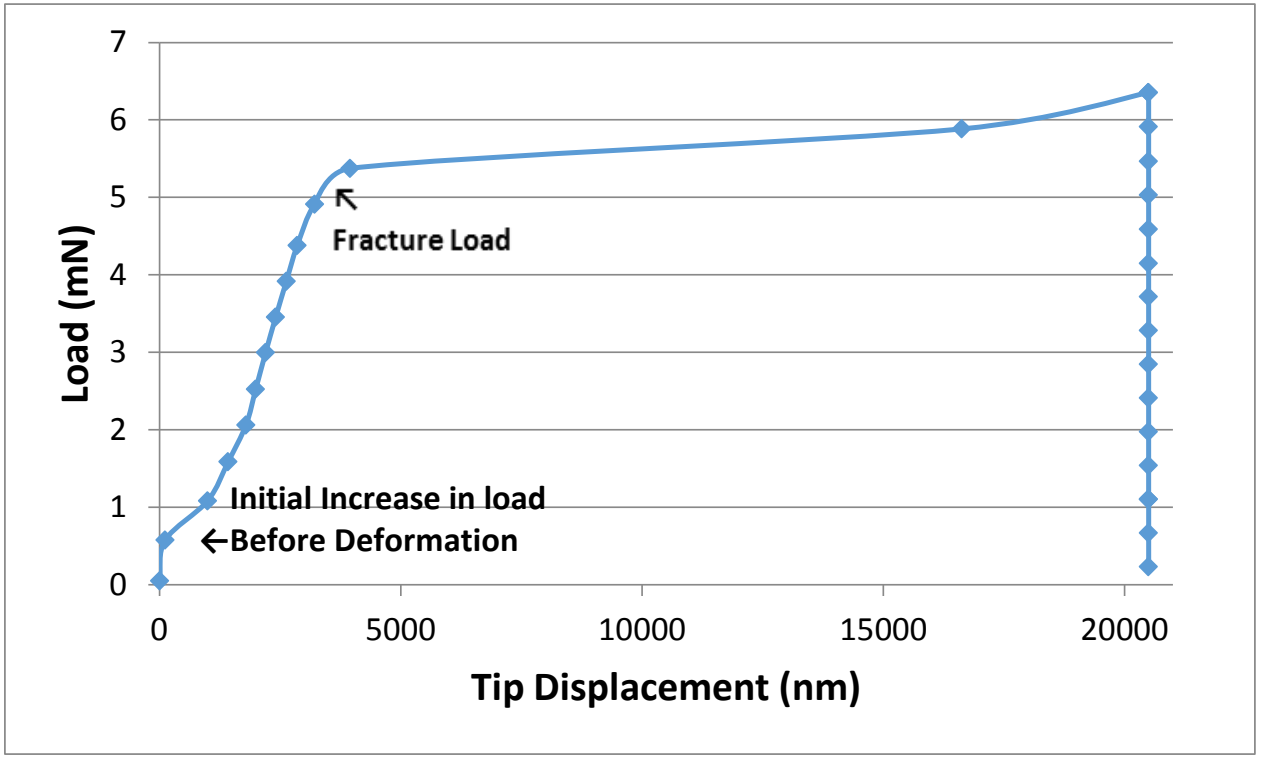

Figure 57: Initial Increase in Load Required Before Deformation Will Occur

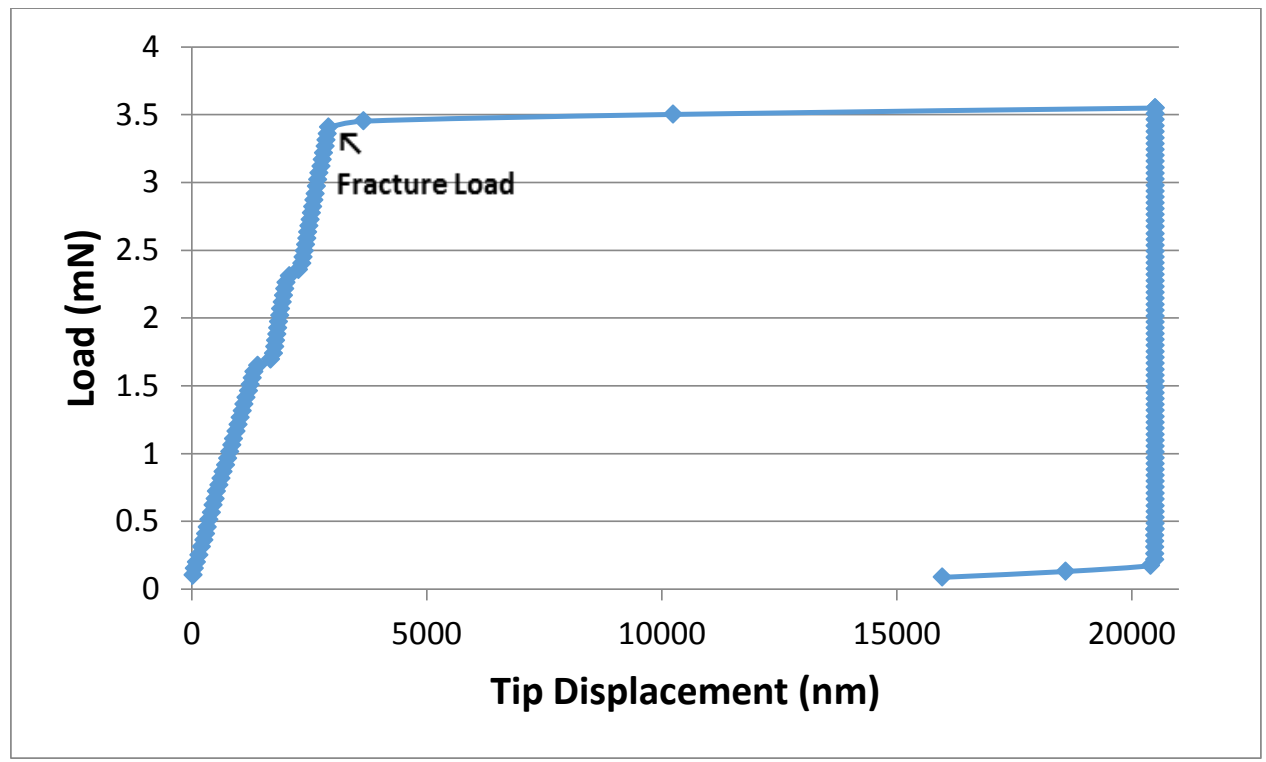

Figure 58: Small Increments Where Deformation Occurs Without an Increase in Load 


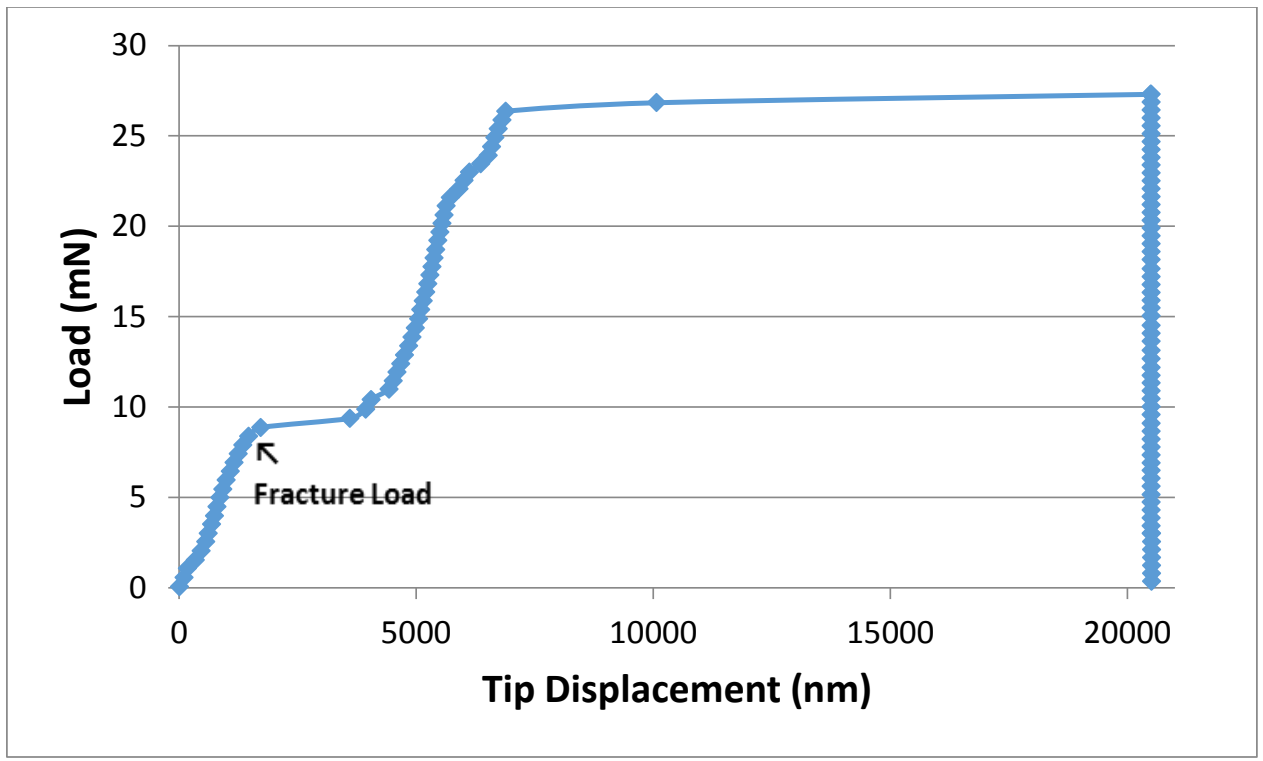

Figure 59: Initial Point of Fracture Followed by Region Requiring More Force to Cause Deformation

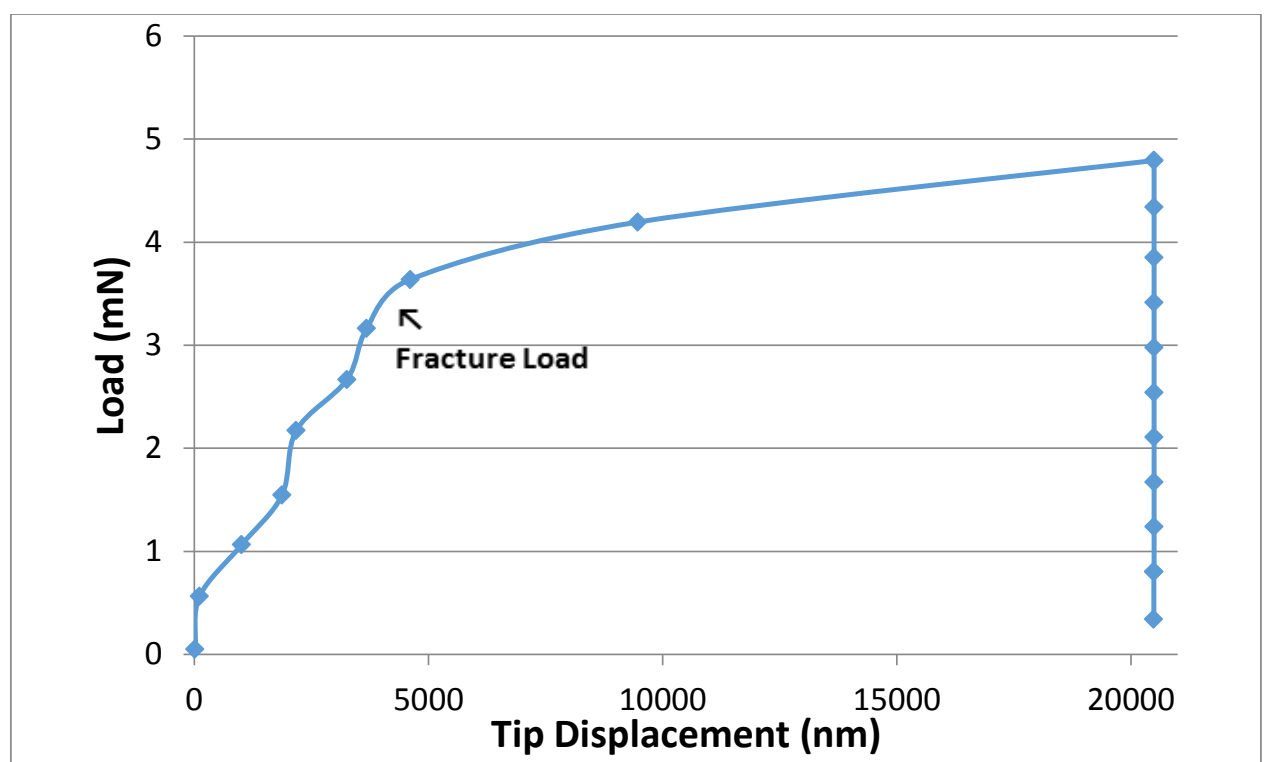

Figure 60: Inconsistent Slope in Region before Fracture 


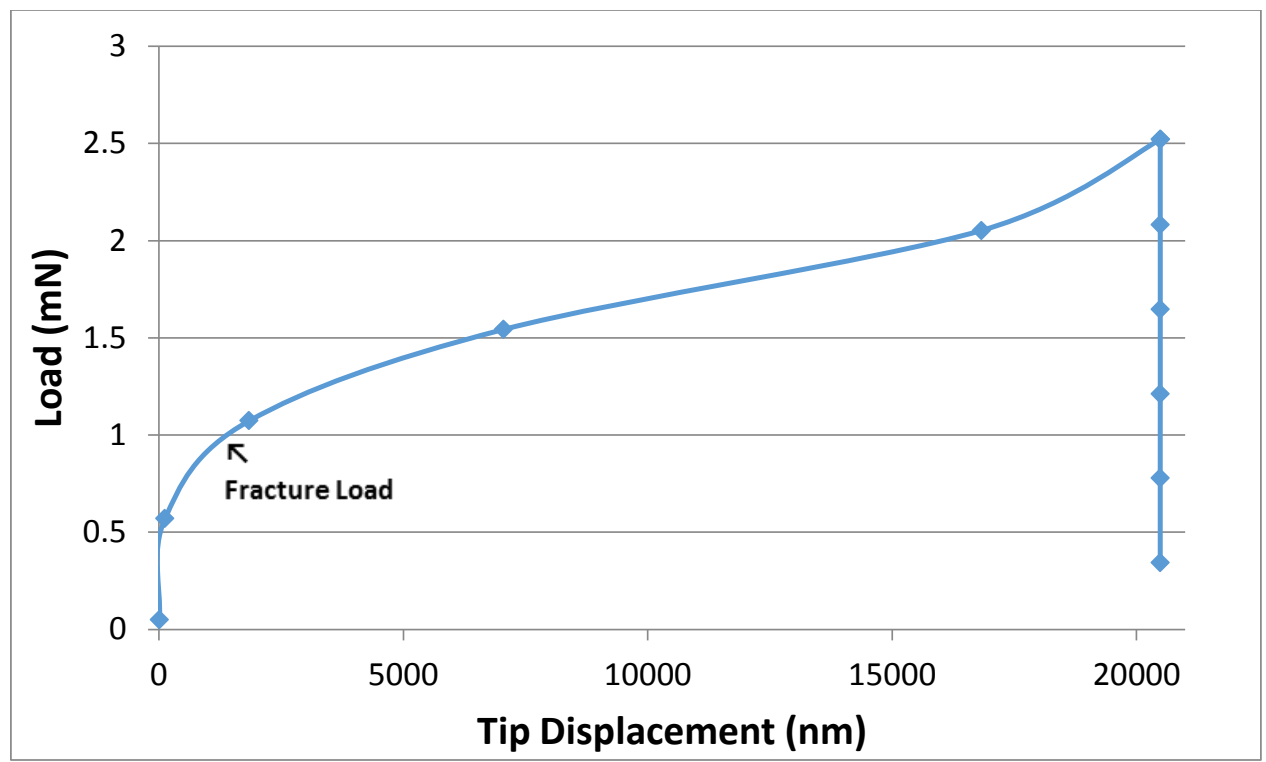

Figure 61: Test Result Where Test Occurred Faster than Output Could be Recorded

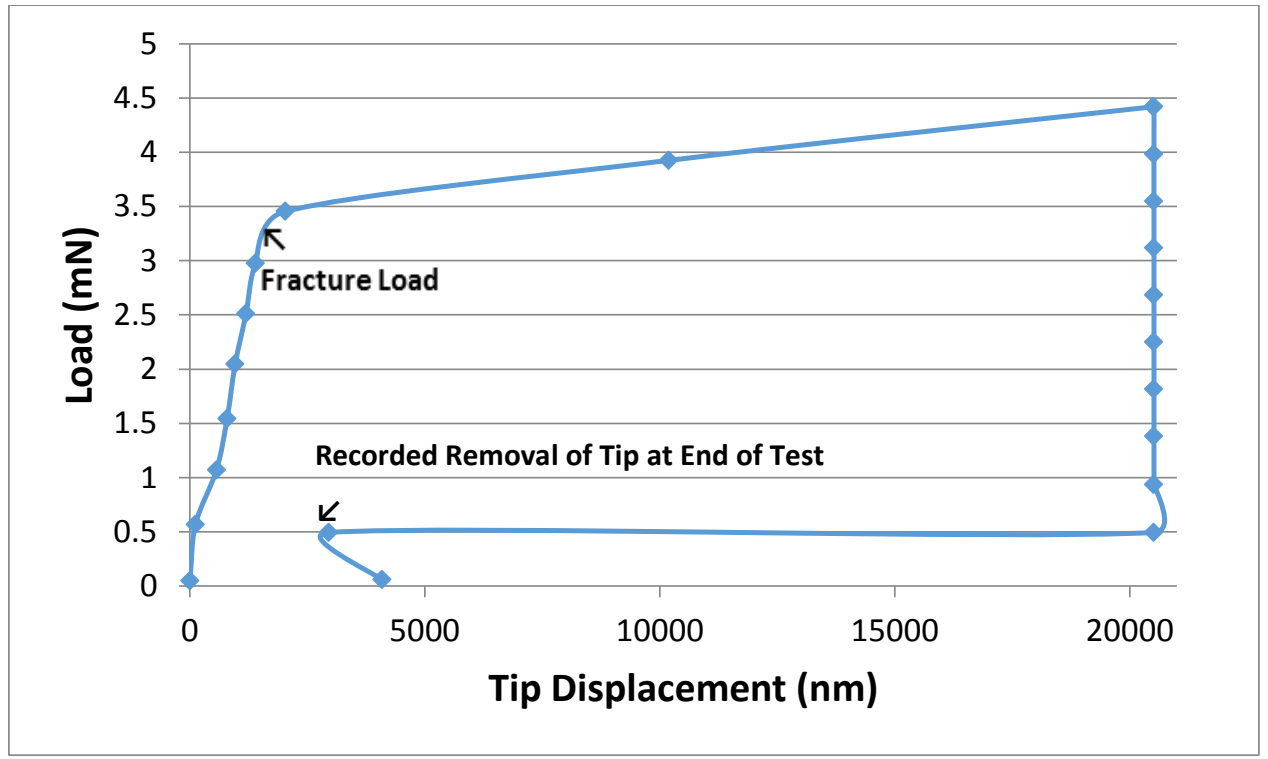

Figure 62: Nanoindenter Recorded Additional Data While Tip Was Removed From Sample 


\section{$\underline{\text { References }}$}

[1] 3M, "3M Glass Bubbles for Buoyancy and Thermal Insulation," 2010. [Online]. Available: http://multimedia.3m.com/mws/media/6313770/3mtm-glass-bubbles-for-buoyancy-andthermal-insulation.pdf. [Accessed 806 2016].

[2] 3M, "Glass Bubbles," 3M, [Online]. Available: http://solutions.3m.com/wps/portal/3M/en_US/EnergyAdvanced/Materials/Product/ ?N=5002440+7570097. [Accessed 806 2016].

[3] G. Wicks, K. Heung and R. Schumacher, "Microspheres and Microworlds," American Ceramic Society Bulletin, pp. 23-27, 062008.

[4] L. K. Heung, G. G. Wicks and R. F. Schumacher, "Encapsulation of Palladium in Porous Wall Hollow Glass Microspheres," Ceramic Transactions, vol. 202, pp. 143-148, 2009.

[5] R. Mohtadi, K. Tange, G. Wicks, K. Heung and R. Schumacher, "A New Way for Storing Reactive Complex Hydrides on Board of Automobiles," Ceramic Transactions, vol. 202, pp. 91-96, 2009.

[6] S. Li, L. Nguyen, H. Xiong, M. Wang, T. C.-C. Hu, J.-X. She, S. M. Serkiz, G. G. Wicks and W. S. Dynan, "Porous-Wall Hollow Glass Microspheres as Novel Potential Nanocarriers for Biomedical Applications," Nanomedicine, vol. 6, pp. 127-136, 2010.

[7] G. Wicks, "Nanostructures and "Nanonothingness" in Unique Glass Microspheres," International Journal of Applied Glass Science, vol. 4, no. 2, pp. 100-104, 2013.

[8] N. Dowling, Mechanical Behavior of Materials; Third Edition, Upper Saddle River: Pearson Education, Inc, 2007.

[9] M. Farooq, "Thick \& Thin Cylinders," [Online]. Available: http://uetmmmksk.weebly.com/uploads/3/6/0/0/3600114/thick_thin_cylinders.pdf. [Accessed $08072016]$.

[10] W. D. Kingery, H. K. Bowen and D. R. Uhlmann, Introduction to Ceramics, John Wiley \& Sons, INC, 1976.

[11] M. Donne, S. Dorner and A. Roth, "Density, thermal expansion coefficient, and viscosity of sodium tetraborate (borax)-UO2 and of sodium metaborate-UO2 solutions at high temperatures," Journal of Nuclear Materials, vol. 118, no. 2-3, pp. 195-205, 1983.

[12] AZO Materials, "Silica - Silicon Dioxide (SiO2)," AZO Network, [Online]. Available: http://www.azom.com/properties.aspx?ArticlelD=1114. [Accessed 806 2016].

[13] R. Mohtadi, T. Matsunaga, K. Heung, R. Schumacher and G. Wicks, "Hollow Glass Microspheres as Micro Media for Complex Hydrogen Storage Compounds," Journal of the SC Academy of Sciences, 
vol. 9, pp. 1-4, 2011.

[14] L. Heung, R. Schumacher and G. Wicks, "Hollow Porous-Wall Glass Microspheres For Hydrogen Storage". United States Patent US 7,666,807 B2, 2322010.

[15] R. Schumacher, G. Wicks and L. Heung, "Hollow Porous-Wall Glass Microspheres for Hydrogen Storage". United States Patent US 2006/0060820 A1, 03232006.

[16] S. Li, H. Xiong, M. Wang, T. C.-C. Hu, J.-X. She, S. M. Serkiz, G. G. Wicks and W. S. Dynan, "Nanocarriers for Biomedical Applications," Journal of the South Carolina Academy of Science, vol. 9, no. 1, pp. 30-32, 2011.

[17] Wyatt Technology, "SEC-MALS," Wyatt Technology Corporation, [Online]. Available: http://www.wyatt.com/solutions/techniques/sec-mals-molar-mass-size-multi-angle-lightscattering.html. [Accessed 1507 2016].

[18] LS Instruments, "Static Light Scattering," LS Instruments, [Online]. Available: www.Isinstruments.ch/technology/static_light_scattering_sls/. [Accessed 296 2016].

[19] Horiba, "Particle Size Analysis Guidebook," [Online]. Available: https://www.horiba.com/fileadmin/uploads/Scientific/eMag/PSA/Guidebook/pdf/PSA_Guideboo k.pdf. [Accessed 242 2016].

[20] "Chapter 22 Standard Plots," NIST, [Online]. Available: www.ncnr.nist.gov/staff/hammouda/distance_learning/chapter_22.pdf. [Accessed 296 2015].

[21] E. Hecht, Optics Forth Edition, Pearson Higher Education, 2001.

[22] Horiba, "La-60 Results Interpretation," [Online]. Available: http://www.horiba.com/fileadmin/uploads/Scientific/Documents/PSA/Products/LA-960/LA960_Result_Interpretation.pdf. [Accessed 2906 2016].

[23] CPS Instruments, "Comparison of Particle Sizing Methods," [Online]. Available: www.cpsinstruments.eu/pdf/Compare\%20Sizing\%20Methods.pdf. [Accessed 307 2016].

[24] 3M, "3M Microspheres Selection Guide," [Online]. Available: http://multimedia.3m.com/mws/media/1300630/3mtm-glass-bubbles-selection-guide.pdf. [Accessed 201622 2].

[25] K. B. Carlisle, K. K. Chawla, G. M. Gladysz and M. Koopman, "Structure and Mechanical Properties of Micro and Macro Balloons: An Overview of Test Techniques," Journal of Materials Science, vol. 41, pp. 3961-3972, 2006.

[26] ASTM, ASTM D 3102-78: Standard Practice for Determination of Isostatic Collapse Strength of Hollow Glass Microspheres, Philadelphia: ASTM, 1978.

[27] D. W. Richerson, Modern Ceramic Engineering, Second Edition, Marcel Dekker, INC, 1992. 
[28] J. B. Wachtman, Mechanical Properties of Ceramics, John Wiley \& Sons, Inc, 1996.

[29] P. W. Bratt, J. P. Cummions and B. D. Spivack, "Mechanical Teesting of Glass Hollow Microspheres," Advances in Materials Characterization, pp. 441-447, 1983.

[30] ASTM Subcommittee D20.10, "Standard Test Methods for Flexural Properties of Unreinforced and Reinforced Plastics and Insulating Materials," ASTM, 2015.

[31] M. Koopman, G. Gouadec, K. Carlisle, K. K. Chawla and G. Gladysz, "Compression Testing of Hollow Microspheres (Microballoons) to Obtain Mechanical Properties," Scripta Materialia, vol. 50, pp. 593-596, 2004.

[32] K. B. Carlisle, M. Koopman, K. K. Chawla, R. Kulkarni, G. M. Gladysz and M. Lewis, "Microstructure and Compressive Properties of Carbon Microballoons," Journal of Materials Science, vol. 41, pp. 3987-3997, 2006.

[33] G. Gouadec, K. Carlisle, K. K. Chawla, M. C. Koopman, G. M. Gladysz and M. Lewis, "NanoCompression of Carbon Micro-Balloons with a Flat-Ended Cylindrical Indenter," in Indentation Techniques in Ceramic Materials CharacteIndentation Techniques in Ceramic Materials, 2003.

[34] K. B. Carlisle, K. K. Chawla, M. Koopman, G. M. Gladysz and M. Lewis, "Uniaxial Compressive Properties of Bismaleamid-Coated Carbon Microballoons," Journal of Cellular Plastics, vol. 43, pp. 417-429, 2007.

[35] W. Callister and D. Rethwisch, Materials Science and Engineering: An Incroduction, John Wiley \& Sons, Inc, 2010.

[36] A. A. Griffith, "The Phenomena of Rupture and Flow in Solids," Philosophical Transactions of the Royal Society of London, Series A, Containing Papers of a Mathematical or Physical Character, vol. 221, pp. 163-198, 1921.

[37] W. Vogel, Glass Chemistry, Springer-Verlag, 1994.

[38] W. Weibull, A Statistical Theory of the Strength of Materials, Stockhold: Barringer and Associates, Inc, 1939.

[39] W. Weibull, "A Statistical Distribution Function of Wide Applicability," Journal of Applied Mechanics, pp. 293-297, 1951.

[40] C. A. Johnson and W. T. Tucker, "Weibull Estimators for Pooled Fracture Data," Life Prediction Methodologies and Data for Ceramic Materials, pp. 250-264, 1994.

[41] R. Danzer, "A General Strength Distribution Function for Brittle Materials," Journal of the European Ceramic Society, vol. 10, pp. 461-472, 1992.

[42] J. Quinn and G. Quinn, "A Practical and Systematic Review of Weibull Statistics for Reporting Strengths of Dental Materials," Dental Materials, vol. 26, no. 2, pp. 135-147, 2010. 
[43] Minitab Inc, "Weibull Distribution," Minitab Inc, 2016. [Online]. Available:

http://support.minitab.com/en-us/minitab/17/topic-library/basic-statistics-and-

graphs/probability-distributions-and-random-data/distributions/weibull-distribution/. [Accessed 8 06 2016].

[44] Minitab, "How to Interpret the Shape Parameter," Minitab, 2016. [Online]. Available: http://support.minitab.com/en-us/minitab/17/topic-library/basic-statistics-andgraphs/probability-distributions-and-random-data/parameters/shape/. [Accessed 806 2016].

[45] "Shape Parameter," Statistics How To, 2016. [Online]. Available: http://www.statisticshowto.com/shape-parameter/. [Accessed 806 2016].

[46] Minitab, "How to Interpret the Scale Parameter," Minitab, 2016. [Online]. Available: http://support.minitab.com/en-us/minitab/17/topic-library/basic-statistics-andgraphs/probability-distributions-and-random-data/parameters/scale/. [Accessed 806 2016].

[47] "Scale Parameter," Statistics How To, 2016. [Online]. Available: http://www.statisticshowto.com/scale-parameter/. [Accessed 806 2016].

[48] "Location and Scale Parameters," NIST, [Online]. Available: http://www.itl.nist.gov/div898/handbook/eda/section3/eda364.htm. [Accessed 806 2016].

[49] K. Trustrum and A. Jayatilaka, "On Estimating the Weibull Modulus for a Brittle Material," Journal of Materials Science, vol. 14, pp. 1080-1084, 1979.

[50] W. Weibull, "Factors Affecting Test Results," in Fatigue Testing and Analysis of Results, Barringer and Associates, Inc, 1961, pp. 94-132.

[51] ASTM Subcommittee C28.01, "C1683-10: Standard Practice for Size Scaling of Tensile Strengths Using Weibull Statistics for Advanced Ceramics," in Book of Standards 15.01, West Conshohocken, ASTM, 2015.

[52] MicroMaterials, "Nanoindentation," MicroMaterials, [Online]. Available: http://www.micromaterials.co.uk/the-nano-test/nanoindentation/. [Accessed 806 2016].

[53] J. H. Rumbarger and R. C. Herrick, "Analysis of the Elastic Contact of a Hollow Ball with a Flat Plate," Franklin Institue Research Laboratories, 1968.

[54] J. H. Chung, Compressive Mechanical Behavior of Hollow Ceramic Spheres and Bonded-Sphere Foams, PH. D. Thesis, Georgia Institute of Technology, 1992.

[55] R. Shorter, J. D. Smith, V. A. Coveney and J. J. C. Busfield, "Axial Compression of Hollow Elastic Spheres," Journal of Mechanis of Materials and Structures, vol. 5, no. 5, pp. 693-705, 2010.

[56] T. H. Elmer, "Porous and Reconstructed Glasses," in Engineered Materials Handbook Volume 4: Ceramics and Glasses, ASM International, 1992, pp. 427-432. 
[57] I. Stefanou and J. Sulem, "Existence of a Threshold for Brittle Grains Crushing Strength: TwoVersus Three-Parameter Weibull Distribution Fitting," Granular Matter, vol. 18, no. 14, pp. 1-10, 2016.

[58] M. H. Dielhof and L. J. M. G. Dortmans, "Fractorgraphy of Borosilicate Glass Tested in Three-Point and Four-Point Bending," Journal of the European Ceramics Society, vol. 12, pp. 215-220, 1993.

[59] s. Feih, A. Thraner and H. Lilholt, "Tensile Strength and Fracture Surface Characterisation of Sized and Unsized Glass Fibers," Journal of Materials Science, vol. 40, pp. 1615-1623, 2005.

[60] AZO Materials, "Borosilicate Glass - Properties of Borosilicate Glass by Goodfellow Ceramics \& Glass Division," AZO Network, [Online]. Available:

http://www.azom.com/article.aspx?ArticlelD=4765. [Accessed 707 2016].

[61] S. K. Rhee, "Surface Energies of Silicate Glasses Calculated from Their Wettability Data," Journal of Materials Science, vol. 12, no. 4., pp. 823-824, 1977.

[62] N. M. Parikh, "Effect of Atmosphere on Surface Tension of Glass," Journal of the American Ceramic Society, vol. 41, no. 1, pp. 18-22, 1958.

[63] Wyatt Technology, "Understanding Dynamic Light Scattering," Wyatt Technology, [Online]. Available: http://wyatt.com/library/theory/dynamic-light-scattering.html. [Accessed 296 2016].

[64] LS Instruments, "Dynamic Light Scattering: Measuring the Particle Size Distribution," LS Instruments, [Online]. Available:

http://www.Isinstruments.ch/technology/dynamic_light_scattering_dls/. [Accessed 296 2016].

[65] UC Davis, "Dynamic Light Scattering," UC Davis, [Online]. Available:

http://chemwiki.ucdavis.edu/Core/Analytical_Chemistry/Instrumental_Analysis/Microscopy/Dyna mic_Light_Scattering. [Accessed 296 2015].

[66] J. H. Rumbarger, R. C. Herrick and P. R. Eklund, "Analysis of the Elastic Contact of a Hollow Ball with a Flat Plate," Transactions of the ASME, pp. 138-142, 1970.

[67] S. Ghosh, M. Tehrani, M. Al-Haik and I. Puri, "Patterning the Stiffness of Elastomeric Nanocomposites by Magnetophoretic Control of Cross-Linking Impeder Distribution," Materials, vol. 8, no. 2, pp. 474-485, 2015.

[68] S. P. Tumoshenko and R. G. Marsters, Theory of Elastic Stability, New York: McGraw-Hill, 1961. 